Zweiter Beratungsgegenstand:

\title{
Verantwortung und Effizienz in der Mehrebenenverwaltung
}

1. Bericht von Prof. Dr. Eckhard Pache, Würzburg

Inhalt

Seite

I. Begriffsbestimmung . . . . . . . . . . . . . 107

1. Europäische Mehrebenenverwaltung . . . . . . . . 108

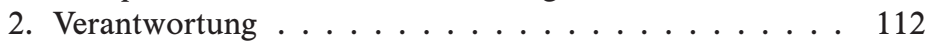

3. Effizienz .................... 115

II. Verantwortungsverteilung in der Mehrebenenverwaltung . . 116

1. Kooperationsbedürfnis .................... 116

2. Kooperationskompetenzen ............. 118

a) Kompetenz zur Verwaltung im Verhältnis

EG-Mitgliedstaaten . . . . . . . . . . 118

b) Kompetenz zur Verwaltung im deutschen

Bundesstaat ....................... 122

c) Kooperationsverbote . . . . . . . . . . . 125

III. Formen kooperativer Verantwortungsverteilung in der

europäischen Mehrebenenverwaltung . . . . . . . . . 126

1. Einzelvollzug . . . . . . . . . . . . . . . . 128

2. Referenzentscheidung . . . . . . . . . . . . . 130

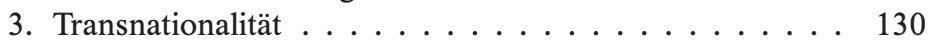

4. Direktvollzug . . . . . . . . . . . . . . 131

5. Netzwerkbildung und Institutionalisierte Kooperation . . 132

6. Wertung . . . . . . . . . . . . . . 135

IV. Demokratische und rechtsstaatliche Verantwortung . . . . 136

1. Anforderungen des Demokratieprinzips . . . . . . . . 137

2. Rechtsstaatliche Anforderungen ... . . . . . . . 141

V. Perspektiven ................... 143 


\section{Begriffsbestimmung}

Der ursprünglich politikwissenschaftliche Begriff des Mehrebenensystems ${ }^{1}$ nimmt ein zentrales Charakteristikum der Verwaltung der Europäischen Union in den Blick: die Funktion des Verwaltens ist nach dem System der Gemeinschaftsverträge und der nationalen Verfassungen der Mitgliedstaaten einer Vielzahl von Verwaltungseinrichtungen der unterschiedlichen Ebenen der EU, der Mitgliedstaaten sowie ihrer subnationalen, regionalen oder lokalen Untergliederungen zugewiesen. Diese Vielzahl von Verwaltungseinrichtungen gemeinsam, ihr Verbund, wird zur Vermeidung unangemessener staats- und verfassungsrechtlicher Einordnungen rechtlich offen als Mehrebenensystem bezeichnet. ${ }^{2}$

\footnotetext{
${ }^{1}$ Zur politikwissenschaftlichen Verwendung des Begriffs vgl. nur F. W. Scharpf Die Politikverflechtungs-Falle: Integration und deutscher Föderalismus im Vergleich, PVS 26 (1985), 323 ff.; M. Jachtenfuchs/B. Kohler-Koch Regieren im dynamischen Mehrebenensystem, in: M. Jachtenfuchs/B. Kohler-Koch (Hrsg.) Europäische Integration, 1996, 30; zur Verbreitung auch in der Rechtswissenschaft und zur gegenwärtigen Beliebtheit etwa jüngst $R$. A. Lorz Autonomie und Bindung der Rechtsetzung in gestuften Rechtsordnungen, DVB1. 2006, 1061; mit Präferenz für den Begriff des „dynamischen Mehrebenensystems“ nunmehr G. F. Schuppert Überlegungen zur demokratischen Legitimation des europäischen Regierungssystems FS Rauschning, 2001, 201 (214 mwN); vgl. auch $R$. Wahl Der Einzelne in der Welt jenseits des Staates, Der Staat 40 (2001), 45 (46ff.).

${ }^{2}$ Der Begriff des Mehrebenensystems wurde innerhalb der europäischen Verfassungsdebatte zentral geprägt von I. Pernice Constitutional Law Implications for a State Participating in a Process of Regional Integration, in: I. Ebsen (Hrsg.) German Reports on Public Law, 1998, 42 ff.; zum Ebenenbegriff als rechtswissenschaftlichem Begriff und seiner Funktion der Ermöglichung national-föderalistisch unvorbelasteter Vergleiche nationaler und übernationaler Rechtsordnungen näher C. Möllers Gewaltengliederung, 2005, $210 \mathrm{ff}$. mwN, der vom Vorliegen einer Ebene im verfassungstheoretischen Sinne ausgeht, „wenn ein bestimmter Rechtsbestand damit beginnt, eigene verfestigte Legitimationsmechanismen zu entwickeln, also seine eigene Rechtserzeugung an die rechtswirksame Anerkennung von individuellen oder demokratischen Akten der Selbstbestimmung zu verknüpfen“, ebd., 213; eher das positive analytische Leistungspotential des Mehrebenenbegriffs betonend F. C. Mayer Europäische Verfassungsgerichtsbarkeit, in: A. von Bogdandy (Hrsg.) Europäisches Verfassungsrecht, 2003, 264 ff. mwN; allgemein zum Europäischen Mehrebenensystem W. Weiß Schnittstellenprobleme des Europäischen Mehrebenenverwaltungsrechts, Die Verwaltung 38 (2005), 517 f.; U. Schliesky Souveränität und Legitimität von Herrschaftsgewalt, 2004, $474 \mathrm{ff}$., im englischsprachigen Schrifttum wird der korrespondierende Begriff „multi-level-governance“ zunehmend auch als Erklärungsmuster für den europäischen Integrationsprozess als solchen verwandt, vgl. für einen Überblick $P$. Craig The Nature of the Community: Integration, Democracy and Legitimacy, in: P. Craig/G. de Burca (Hrsg.) The Evolution of EU Law, 1999, 16 ff. Nach der Definition des Begriffes „multi-level-governance“ von N. Bernard
} 
Werden an dieses Mehrebenensystem die Maßstäbe von Verantwortung und Effizienz angelegt, so scheint mit ihnen einerseits nach der systemimmanenten Aufgaben- und Funktionsverteilung und andererseits nach der Einhaltung grundlegender demokratischer und rechtsstaatlicher Anforderungen gefragt. Doch zunächst präziser: Was genau ist die europäische Mehrebenenverwaltung, ${ }^{3}$ und welche Rolle spielen Verantwortung und Effizienz in ihr?

\section{Europäische Mehrebenenverwaltung}

In der Gründungsphase der Europäischen Gemeinschaften schienen die Struktur und die Ausgestaltungsprinzipien der Verwaltung des Gemeinschaftsraumes ${ }^{4}$ eindeutig. ${ }^{5}$ EG-Recht sollte auf der Grundlage eines

Multilevel Governance in the European Union, 2002, 8, beschreibt das Konzept „multi-level-governance“ „any case of decisionmaking which involves several actors situated at different levels, such as Community and national levels“.

3 Wegen der Vielgestaltigkeit vorstellbarer Mehrebenensysteme wird nicht die grundsätzliche Eignung irgendeines, sondern die konkrete Eignung des existenten Modells europäischer Verwaltung im Mehrebenensystem für die Verwirklichung von Verantwortung und Effizienz analysiert; auf der Grundlage dieser Analyse sollen Vorstellungen für eine wert- oder prinzipiengerechte Modellierung und künftige Ausgestaltung gesucht werden; allgemein zu den Legitimationsstrukturen in Mehrebenen-Rechtsordnungen Möllers Gewaltengliederung (Fn. 2), $210 \mathrm{ff}$, , wobei dort eine Untersuchung des Begriffs der Ebene als Kategorie des übernationalen Rechtsvergleichs föderaler Ordnungen erfolgt. Außerdem hierzu Schliesky Souveränität (Fn. 2), 479 ff., der von den zahlreichen Erscheinungsformen ,gestufter Staatlichkeit“" spricht.

${ }^{4}$ Schwierigkeiten bestanden allerdings - und bestehen immer noch -, im institutionellen System der europäischen Ebene die Funktion der Verwaltung gewaltenteilend eindeutig zu verorten; zum Problem der Einordnung der Europäischen Gemeinschaftsstruktur mit Hilfe staatsrechtlicher Grundsätze, speziell des Gewaltenteilungsgrundsatzes J. Schwarze Europäisches Verwaltungsrecht, 2. Aufl. 2005, 193 ff. mwN; zu den Defiziten bezüglich der supranationalen Ebene aufgrund des Abbaus der Gewaltenteilung Schliesky Souveränität (Fn. 2), 403 ff.; zur Schwierigkeit der Verortung der Exekutive auf europäischer Ebene Möllers Gewaltengliederung (Fn. 2), $270 \mathrm{ff}$. Zur Rolle der Kommission als Gubernative im Bereich des Wettbewerbsrechts ders. ebd., 335.

${ }^{5}$ Zur Durchbrechung des ursprünglichen Bildes einer Aufgabenverteilung zwischen gemeinschaftlicher Normsetzung und mitgliedstaatlichem Vollzug $J$. Gundel 33 Verwaltung, in: R. Schulze/ M. Zuleeg (Hrsg.) Europarecht Handbuch für die deutsche Rechtspraxis, 2006, Rn. 3 ff.; zur gestiegenen Beteiligung der Gemeinschaft am Vollzug D. König § 2 Gesetzgebung, in: Schulze/Zuleeg, Rn. 82 ff.; zur Intensivierung der Mitwirkung der Mitgliedsstaaten am Gesetzgebungsverfahren vgl. auch $G$. Winter Kompetenzverteilung und Legitimation in der Europäischen Mehrebenenverwaltung, EuR $2005,255$. 
strikten Trennungsprinzips ${ }^{6}$ in dualistischer Grundstruktur entweder direkt-gemeinschaftsunmittelbar durch die Organe der EG oder indirekt-mittelbar durch die Verwaltungen der Mitgliedstaaten jeweils eigenständig vollzogen werden. ${ }^{7}$

Dabei sollte die Aufgabe des Verwaltens überwiegend den Verwaltungen der Mitgliedstaaten obliegen, ${ }^{8}$ die je für sich in strikter organisatorischer, personeller und funktionaler Trennung von den übrigen Mitgliedstaaten und den Gemeinschaftsorganen die Rechtsordnung und die Gemeinwohlbelange der Europäischen Union umsetzen und verwirklichen sollten. ${ }^{9}$ Der daneben in Einzelbereichen erfolgende direkte oder gemeinschaftsunmittelbare Vollzug des Gemeinschaftsrechts durch die Organe der EG sollte demgegenüber die strukturell rechtfertigungsbedürftige Ausnahme bilden. ${ }^{10}$

${ }^{6}$ Zum Trennungsprinzip als einem der beiden für die Verwaltung des Gemeinschaftsraums konstitutiven Elemente näher E. Schmidt-Aßmann Das allgemeine Verwaltungsrecht als Ordnungsidee, 2. Aufl. 2004, $381 \mathrm{ff}$. mwN. Zur traditionellen Konzeption getrennter Verwaltungen G. Sydow Verwaltungskooperation in der Europäischen Union, 2004, 1 ff.; auch hierzu S. Kadelbach Allgemeines Verwaltungsrecht unter Europäischem Einfluss, 1999, 16ff.; W. Kahl Europäische und nationale Verwaltungsorganisation, Die Verwaltung 29 (1996), 349.

${ }^{7} \mathrm{Zu}$ Grundstruktur und Formen des dualistischen Verwaltungsvollzugs des EGRechts vgl. nur H.-W. Rengeling Rechtsgrundsätze beim Verwaltungsvollzug des Europäischen Gemeinschaftsrechts, 1977, 9 ff. mwN; E. Pache Der Schutz der finanziellen Interessen der Europäischen Gemeinschaften, 1994, 74 ff. mwN; H. P. Nehl Europäisches Verwaltungsverfahren und Gemeinschaftsverfassung, 2002, 30f.; Schwarze Europäisches Verwaltungsrecht (Fn. 4), 25 ff.; J. Suerbaum Die Kompetenzverteilung beim Verwaltungsvollzug des Europäischen Gemeinschaftsrechts in Deutschland, 1998, $116 \mathrm{ff}$; A. Hatje Die gemeinschaftsrechtliche Steuerung der Wirtschaftsverwaltung, 1998, $43 \mathrm{ff}$; W. Pühs Der Vollzug von Gemeinschaftsrecht, 1997, 73 ff; Gundel Verwaltung (Fn. 5), Rn. $91 \mathrm{ff}$.

${ }^{8}$ Ausführliche Darstellung bei Rengeling Rechtsgrundsätze (Fn. 7), 26 ff. mwN.

${ }_{9}$ Als Grundlage des mitgliedstaatlichen Vollzugs wird die Verpflichtung zur Gemeinschaftstreue aus Art. 10 EGV angesehen; die unterschiedlichen aus der Pflicht zur Gemeinschaftstreue ableitbaren Handlungspflichten der einzelnen Staatsfunktionen behandelt etwa R. Streinz in: ders. (Hrsg.) EUV/EGV, Art. 10 Rn. 19 ff. mwN. Über das aus der Gemeinschaftstreue für die Mitgliedsstaaten teilweise folgende enorme Konfliktpotenzial bezüglich des mitgliedstaatlichen Rechts D. H. Scheuing Europarechtliche Impulse für innovative Ansätze im deutschen Verwaltungsrecht, in: W. HoffmannRiem/E. Schmidt-Aßmann (Hrsg.) Innovation und Flexibilität des Verwaltungshandelns, 1994, 289 (293f.); zur Unionstreue im Allgemeinen T. Marauhn § 7 Unionstreue, in: Schulze/Zuleeg (Fn. 5), Rn. $1 \mathrm{ff}$.

$10 \mathrm{Vgl}$. nur E. Schmidt-Aßmann Verwaltungskooperation und Verwaltungskooperationsrecht in der Europäischen Gemeinschaft, EuR 1996, 270 mwN. 
Dieses traditionelle Bild ${ }^{11}$ erfasst die Realität der Mehrebenenverwaltung in der Europäischen Union heute nicht mehr zutreffend. Phänomenologisch besteht zwar sowohl die organisatorische Trennung der europäischen und der mitgliedstaatlichen Verwaltungen als auch der Vorrang des mitgliedstaatlichen Vollzuges des Europarechts fort. ${ }^{12}$ Allerdings ist die Eigenverwaltung der EG in den letzten Jahren ganz erheblich ausdifferenziert worden. ${ }^{13}$ Zahlreiche europäische Agenturen, Ämter und Stellen sind als verselbständigte oder unselbständige Organisationseinheiten auf der Ebene der Europäischen Gemeinschaften gegründet ${ }^{14}$ und

${ }^{11}$ Zusammenfassend zu traditionellen Vorstellungen und Konzeptionen europäischer Verwaltung Sydow Verwaltungskooperation (Fn. 6), 1 ff. mwN, dort auch zu den Konzeptionen eines sog. „Vollzugsföderalismus“ oder eines „system of divided administration"; in Betracht kommt auch eine Bezeichnung als Exekutivföderalismus. Von einem „exekutiv-föderalistischen System“ bzw. von Vollzugsföderalismus spricht auch I. Pernice Kompetenzabgrenzung im Europäischen Verfassungsverbund, JZ 2000, 866 (871).

12 Die mitgliedstaatlichen Verwaltungen bleiben die Basis der Europäischen Verwaltung, erfüllen auch weiterhin die meisten Verwaltungsaufgaben und erledigen die meisten Verwaltungsvorgänge, so dass von einem Vorrang dezentraler, mitgliedstaatlicher Aufgabenwahrnehmung als Realbefund gesprochen werden kann, so E. Schmidt-Aßmann $§ 5$ Verfassungsprinzipien für den europäischen Verwaltungsverbund, in: W. Hoffmann-Riem/E. Schmidt-Aßmann/A. Voßkuhle (Hrsg.) Grundlagen des Verwaltungsrechts, Bd. I, 2006, Rn. 19.

13 Ausführlicher Sydow Verwaltungskooperation (Fn. 6), 63 ff. mwN; H. SchulzeFielitz Die Verwaltung im europäischen Verfassungsgefüge, in: W. Erbguth/J. Masing (Hrsg.) Verwaltung unter dem Einfluss des Europarechts, 2006, 91 (96ff. mwN); vgl. auch $R$. Priebe Die Übertragung von Entscheidungsbefugnissen vertragsfremder Einrichtungen im Recht der Europäischen Gemeinschaften, 1979, $15 \mathrm{ff}$.; instruktiv auch Streinz EUV/EGV (Fn. 9), Art. 7 Rn. 29 ff. mwN; zur Wirkungsweise des EG-Eigenverwaltungsrechts $S$. Hegels EG-Eigenverwaltungsrecht und Gemeinschaftsverwaltungsrecht, 2001, 48ff.; zu den Regeln des EG-Eigenverwaltungsrechts Gundel Verwaltung (Fn. 5), Rn. $180 \mathrm{ff}$.

${ }^{14}$ Zur Ausdifferenzierung der Gemeinschaftsverwaltung durch Ämter und Agenturen der Gemeinschaft Gundel Verwaltung (Fn. 5), Rn. 25 ff.; über die bisherigen Entwicklungsphasen bei der Gründung rechtsfähiger Einrichtungen ohne ausdrückliche vertragliche Ermächtigung $M$. Berger Vertraglich nicht vorgesehene Einrichtungen des Gemeinschaftsrechts mit eigener Rechtspersönlichkeit, 1999, $31 \mathrm{ff}$. Einen umfänglichen Überblick über kooperative Verwaltungsstrukturen gibt $H$. $-H$. Vogel Verwaltungsstrukturen der Europäischen Union: Kooperationsverwaltung durch die Schaffung von Agenturen, in: J. Iliopoulos-Strangas/H. Bauer (Hrsg.) Die Neue Europäische Union, 2006, $199 \mathrm{ff}$. Zu den Anfängen der Binnendifferenzierung der Gemeinschaftsverwaltung schon M. Hilf Die abhängige Juristische Person des Europäischen Gemeinschaftsrechts, ZaöRV 36 (1976), 551 ff. Allgemein über die verschiedenen Modelle Europäischer Agenturen und deren Entwicklung in den letzten Jahren M. H. Koch Mittelbare Gemeinschaftsverwaltung in der Praxis, EuZW 2005, $455 \mathrm{ff}$. 
auf vielfältige Weise in die Verwaltung des Gemeinschaftsraumes eingebunden worden. ${ }^{15}$

Vor allem aber ist der mitgliedstaatliche Vollzug des Rechts der Europäischen Union heute eingebunden in zahlreiche Strukturformen realen Kooperationshandelns, ${ }^{16}$ das vielfältig verflochten und multidirektional zwischen den mitgliedstaatlichen Verwaltungen und der Kommission erfolgt. Nicht mehr die organisatorische Trennung, sondern die informale und die formale Kooperation sind die zentralen Paradigmata der europäischen Verwaltung. Die gemeinschaftlichen und die mitgliedstaatlichen Verwaltungsinstanzen bilden einen Informations-, Handlungsund Kontrollverbund, ${ }^{17}$ der im Wege informationeller, prozeduraler und institutioneller Zusammenarbeit das Gemeinschaftsrecht verwaltet. ${ }^{18}$

${ }^{15}$ Die Ausdifferenzierung der Eigenverwaltung der EG spiegelt organisatorisch die verstärkte Wahrnehmung von Verwaltungsaufgaben durch die europäische Ebene des Mehrebenensystems wider; für einen aktuellen Überblick über die so genannten dezentralen Einrichtungen der Europäischen Union vgl. http://europa.eu/agencies/ index_de.htm; hierzu auch W. Kilb Europäische Agenturen und ihr Personal - die groBen Unbekannten?, EuZW 2006, 268 ff.; M. Fehling Europäische Verkehrsagenturen als Instrumente der Sicherheitsgewährleistung und Marktliberalisierung insbesondere im Eisenbahnwesen, EuR 2005 Beiheft 2, $41 \mathrm{ff}$.

16 R. Pitschas Europäisches Verwaltungsverfahrensrecht und Handlungsformen der gemeinschaftlichen Verwaltungskooperation, in: H. Hill/R. Pitschas (Hrsg.) Europäisches Verwaltungsverfahrensrecht, 2004, 301 (324); Sydow Verwaltungskooperation (Fn. 6), 3.

17 So E. Schmidt-Aßmann Europäische Verwaltung zwischen Kooperation und Hierarchie, FS Steinberger, 2002, 1375 ff.; ders. Strukturen Europäischer Verwaltung und die Rolle des Europäischen Verwaltungsrechts, in: A. Blankenagel/I. Pernice/H. SchulzeFielitz (Hrsg.) Verfassung im Diskurs der Welt, FS Häberle, 2004, 395 ff.; ders. Einleitung: Der Europäische Verwaltungsverbund und die Rolle des Verwaltungsrechts, in: ders./B. Schöndorf-Haubold (Hrsg.) Der europäische Verwaltungsverbund, 2005, 1 (6); T. Groß Exekutive Vollzugsprogrammierung durch tertiäres Gemeinschaftsrecht?, DÖV 2004, 20 ff.; ders. Die Kooperation zwischen europäischen Agenturen und nationalen Verwaltungen, EuR 2005, $54 \mathrm{ff}$.

18 Erst durch Kooperation im Verbund der nationalen und gemeinschaftlichen Institutionen können sich die beteiligten Verwaltungen durch informationelle Kooperation die erforderlichen Informationen erschließen, durch prozedurale Kooperation gegenseitige Unterrichtung, Abstimmung und gemeinsame Verfahrensführung leisten und in entsprechenden Gremien auch institutionell abgesichert kooperieren, so mit konkreten Beispielen Schmidt-Aßmann Verwaltungsverbund (Fn. 17), 6. Grundlegend über die Verwaltungskooperation Scheuing Europarechtliche Impulse (Fn. 9), 289 (294 ff.); P. M. Huber Das Kooperationsverhältnis von Kommission und nationale Verwaltungen beim Vollzug des Unionsrechts, FS Brohm 2002, 127 ff.; speziell zur Verwaltungskooperation bezüglich der nationalen Regulierungsbehörden K. H. Ladeur/C. Möllers Der europäische Regulierungsverbund der Telekommunikation im deutschen Verwaltungsrecht, DVB1. 2005, 525 (526f.). 
In Verwirklichung des europarechtlichen Kooperationsprinzips ${ }^{19}$ sind also die organisatorisch getrennten Ebenen der europäischen Mehrebenenverwaltung funktional im Interesse einer effektiven Gemeinschaftsverwaltung vielfältig durch Kooperation verflochten. ${ }^{20}$

\section{Verantwortung}

Was bedeutet nun in diesem europäischen Mehrebenensystem Verantwortung, ${ }^{21}$ und wo ist sie zu verorten?

\footnotetext{
${ }^{19}$ Zum Kooperationsprinzip als allgemeiner Leitidee der EU als kooperativer Verfassungs- und Verwaltungsgemeinschaft Pitschas Europäisches Verwaltungsverfahrensrecht (Fn. 16), 301 (304f. mwN); das Kooperationsprinzip trägt dem Umstand Rechnung, dass ungeachtet der organisatorischen Trennung der Verwaltungen die Europäischen Gemeinschaften von Anfang an nicht nur Rechtsgemeinschaften, sondern ebenso auch Verwaltungsgemeinschaften waren, so ausdrücklich Schmidt-Aßmann Strukturen (Fn. 17), 395 (396); ders. Verfassungsprinzipien (Fn. 12), Rn 16; diese Eigenschaft als Verwaltungsgemeinschaften hat erst seit Mitte der neunziger Jahre des vergangenen Jahrhunderts größere Beachtung gefunden, vgl. zur zunehmenden Wahrnehmung der EG als Verwaltungsgemeinschaft auf europäischer Ebene den Sutherland-Bericht von 1993 sowie die Mitteilung der Kommission an den Rat und das Europäische Parlament über die Entwicklung der Zusammenarbeit der Verwaltungen bei der Anwendung und Durchsetzung des Gemeinschaftsrechts für den Binnenmarkt, KOM (94) 29 endg., BR-Drs. 241/94; zur erst späten Wahrnehmung der Europäischen Gemeinschaften als Verwaltungsgemeinschaften auch Schmidt-Aßmann Verwaltungskooperation (Fn. 10), 270 (270f. mwN) sowie ders. Verfassungsprinzipien (Fn. 12), Rn. 16; insgesamt war Verwaltungskooperation von Anfang an und ist heute mehr denn je ein tragendes Element und zugleich eine unabdingbare Voraussetzung für das praktische Funktionieren des europäischen Integrationsprozesses, deutlich in diesem Sinne auch R. Priebe Handlungsformen für Verwaltungskooperation im europäischen Staatenverbund, in: Hill/Pitschas (Fn. 16), 337 ff. Über den Austausch von Informationen als Mindestinhalt jeder Verwaltungskooperation J. Sommer Verwaltungskooperation am Beispiel administrativer Informationsverfahren im Europäischen Umweltrecht, 2003, 74 ff.

${ }^{20}$ Winter Kompetenzverteilung (Fn. 5), 255 (258f.) benennt als Ursachen der Kooperation die Unterschiedlichkeit der nationalen Verwaltungen, die zu der sehr fortgeschrittenen Harmonisierung der rechtlichen Bedingungen in Spannung stehe, und nimmt damit inhaltlich Gesichtspunkte der Effizienz und Gleichmäßigkeit des Verwaltungsvollzuges in den Blick.

${ }^{21}$ Diese Frage stellt sich, da der Begriff der Verantwortung nicht nur in der Rechtswissenschaft, sondern ebenso in Politikwissenschaft, Philosophie und Theologie verbreitete Verwendung findet; zu den ethymologischen und historischen Wurzeln des Begriffs Verantwortung und zu seiner ursprünglichen Verwendung in der christlichen Ethik und in der Rechtssprache instruktiv $M$. Führ Eigenverantwortung im Rechtsstaat, 2003, 43 ff. mwN; zusammenfassend zu Sinn, Geschichte und Grund von Verantwortung vgl. Görres-Gesellschaft (Hrsg.) Staatslexikon, Bd. 5, 1989, Stichwort „Verantwortung"; auf die ethischen und philosophischen Konnotationen des Begriffs der Verant-
} 
Verantwortung gilt zwar als Schlüssel- und Leitbegriff mit erheblichem analytischem Potential und beträchtlicher Problemlösungskapazität, 22 der Begriff ist allerdings bis heute in hohem Maße kontextabhängig und ausfüllungsbedürftig geblieben. ${ }^{23}$

wortung und seine Zuordnung zum Bereich des Sittlichen weist deutlich D. Wilke Über Verwaltungsverantwortung, DÖV 1975, 509 mwN hin. Für eine instruktive Analyse des korrespondierenden englischen Begriffes „accountability“ siehe C. Harlow Accountability in the European Union, 2002, 6ff.

22 Ausführlich der Überblick bei $H$. Dreier Verantwortung im demokratischen Verfassungsstaat, ARSP Beiheft 74 (2000), 9 (9ff. mwN); vgl. weiter etwa G. F. Schuppert Verwaltungswissenschaft, 2000, 400: „Die gleich näher zu erörternden Konzepte einer abgestuften Verwaltungsverantwortung (...) verweisen notwendig auf den vorausliegenden Begriff der Verwaltungsverantwortung, der sich mehr und mehr (...) als Schlüsselbegriff für die analytische Durchdringung einer im Kooperationsspektrum staatlicher und privater Aufgabenerfüllung handelnden Verwaltung erweist“.

23 So ausdrücklich $A$. Voßkuhle Beteiligung Privater an der Wahrnehmung öffentlicher Aufgaben und staatliche Verantwortung, VVDStRL 62 (2003), 266 (270 Fn. 9 mwN); skeptisch gegenüber der Eignung des Begriffs der Verantwortung für die verwaltungsrechtsdogmatische Diskussion H.C. Röhl Verwaltungsverantwortung als dogmatischer Begriff?, Die Verwaltung 1999 Beiheft 2, 33 (33, 35 ff.); teilweise wird dem Begriff der Verantwortung deshalb mangelnde Trennschärfe oder begriffliche Beliebigkeit vorgeworfen, vgl. Nachweise bei Dreier Verantwortung (Fn. 22), 11; Führ Eigenverantwortung (Fn. 21), 43 mwN spricht von einer uneinheitlichen, oftmals diffusen Begrifflichkeit mit teils normativen, teils deskriptiven Bedeutungsgehalten; kritisch zum Wert des Begriffs der Verantwortung für die Erfassung der öffentlich-rechtlichen Funktionenordnung bereits $F$. Ossenbühl Die Zustimmung des Bundesrates beim Erlass von Bundesrecht, AöR 99 (1974), 369 (407); Staatsrechtslehre und Staatstheorie charakterisieren Verantwortung überwiegend als heuristischen Begriff, der weder ein konturiertes Rechtsinstitut noch einen eindeutig definierten Gegenstand bezeichnet, so schon E. Schmidt-Aßmann Verwaltungsverantwortung und Verwaltungsgerichtsbarkeit, VVDStRL 34 (1976), 221 (227ff. mwN), der dort den Begriff der Verwaltungsverantwortung als heuristischen Begriff zur Kennzeichnung eines differenzierten Ausgleichssystems definiert und auf Verfassungsebene primär der Gewaltenteilung zugeordnet hat; ausführlich zu den prozeduralen und materiellen Dimensionen der Verwaltungsverantwortung auch $R$. Scholz Verwaltungsverantwortung und Verwaltungsgerichtsbarkeit, VVDStRL 34 (1976), 145 (149ff. mwN); grundlegend zur Verwaltungsverantwortung Wilke Verwaltungsverantwortung (Fn. 21), $509 \mathrm{ff}$; t teilweise wird Verantwortung in ihrer spezifischen Ausprägung als Verwaltungsverantwortung schlicht als zusammenfassendes Kürzel für die umfassende Aufgabe und Rolle der Verwaltung bei der Gemeinwohlverwirklichung im modernen sozialen Rechtsstaat verstanden, in diesem Sinne etwa R. Pitschas Verwaltungsverantwortung und Verwaltungsverfahren. Strukturprobleme, Funktionsbedingungen und Entwicklungsperspektiven eines konsensualen Verwaltungsrechts, 1990, 10 sowie in Anlehnung hieran Schuppert Verwaltungswissenschaft (Fn. 22), $400 \mathrm{ff}$. mwN, teilweise werden demgegenüber die einzelnen Aspekte von Verwaltungsverantwortung entfaltet, so etwa die grundlegende Differenzierung von Schmidt-Aßmann Verwaltungsverantwortung, $232 \mathrm{ff}$, der für die Verantwortung der Ver- 
In öffentlich-rechtlichen Untersuchungszusammenhängen werden zumindest drei Dimensionen von Verantwortung unterschieden: ${ }^{24}$ In der Staatsrechtslehre ist der Begriff zunächst als Verwaltungsverantwortung mit Blick auf die Gewaltenteilung zwischen Exekutive und Judikative verwendet worden. ${ }^{25}$ Statt dieser gewaltenteilenden Perspektive steht im Rahmen der Staatsaufgabendiskussion eher die Verantwortungsteilung zwischen Staat und Gesellschaft im Vordergrund. ${ }^{26}$

Innerhalb der Mehrebenenverwaltung der Europäischen Union dagegen geht es bei Verantwortung einerseits um die Zuweisung von Handlungszuständigkeiten und Handlungsbefugnissen $\mathrm{zu}$ den verschiedenen Ebenen und anderseits um die demokratische und rechtsstaatliche $\mathrm{Zu}$ rechnung und Kontrolle administrativer Handlungen. Es geht darum, wie die Kompetenzen und Befugnisse für Verwaltungshandeln in der Europäischen Union verteilt sind und wer die Ergebnisse dieses Verwaltungshandelns demokratisch und rechtsstaatlich ${ }^{27} \mathrm{zu}$ vertreten hat. ${ }^{28}$

waltung innerhalb der Funktionenordnung des Staates zwischen Vollzugsverantwortung, Entfaltungsverantwortung, Verantwortung für Programmverwirklichung, Initiativverantwortung und Gesamtverantwortung unterscheidet; nicht in den Blick genommen wird dabei die Verantwortung im Verhältnis zu nicht-staatlichen Akteuren, vgl. hierzu unter dem Begriff der Verantwortungsstufung Schuppert Verwaltungswissenschaft (Fn. 22), 402 ff. mwN.

${ }^{24}$ Hierzu Schmidt-Aßmann Ordnungsidee (Fn. 6), 170f. mwN, nach dessen Einteilung sich Verantwortung jedenfalls auf das Verhältnis von staatlicher Aufgabenerfüllung zu gesellschaftlicher Aufgabenwahrnehmung, das Verhältnis der Staatsfunktionen zueinander und schließlich auf das inneradministrative Verhältnis mehrerer an der Erfüllung von Verwaltungsaufgaben beteiligter Verwaltungseinheiten beziehen kann; eher einschränkend H. Schulze-Fielitz $§ 12$ Grundmodi für die Aufgabenwahrnehmung, in: Hoffmann-Riem/Schmidt-Aßmann/Voßkuhle (Fn. 12), Rn. 148: „Rechtlich kann Verantwortung nichts anderes sein als das, was das Grundgesetz und/oder die jeweiligen Fachgesetze an Aufgaben und Befugnissen für die Verwaltung vorsehen“, allerdings ist diese eingrenzende Begriffsbestimmung bezogen auf die Gemeinsamkeiten der unterschiedlichen Verwaltungsverantwortung in den verschiedenen Erscheinungsformen der Wahrnehmung von Verwaltungsaufgaben.

25 Schmidt-Aßmann Verwaltungsverantwortung (Fn. 23), 232ff.; Scholz Verwaltungsverantwortung (Fn. 23), $145 \mathrm{ff}$.

${ }^{26}$ Hierzu ausführlich $A$. Voßkuhle Staatliche Verantwortung (Fn. 23), 266ff.; vgl. auch Schuppert Verwaltungswissenschaft (Fn. 22), $400 \mathrm{ff}$. mwN.

27 Diese Aspekte der Verantwortung im Mehrebenensystem betont H.C.Röhl Verantwortung und Effizienz in der Mehrebenenverwaltung, DVB1. 2006, 1070.

28 Verantwortung in der europäischen Mehrebenenverwaltung ist damit ein $\mathrm{Zu}-$ ordnungsbegriff und meint die differenzierte Zuweisung von Handlungszuständigkeiten, Handlungsbefugnissen, Handlungsaufträgen und Handlungslegitimation an die verschiedenen Einrichtungen und Institutionen der vielgestaltigen vertikalen und horizontalen Verwaltungskooperation in Europa sowie die demokratische und rechtsstaatliche Zurechnung von Handlungsergebnissen dieses Verwaltungsverbundes. Problema- 


\section{Effizienz}

Spielt nun Effizienz für die Verantwortungsverteilung in der Mehrebenenverwaltung eine Rolle?

Effizienz bezeichnet grundsätzlich das Verhältnis zwischen eingesetztem Aufwand und angestrebtem Erfolg und zielt auf eine optimale Zweck-Mittel-Relation. ${ }^{29}$ Entweder soll ein angestrebtes Ziel mit möglichst geringen Mitteln oder es soll mit vorhandenen Mitteln der größtmögliche Erfolg erreicht werden. ${ }^{30}$ Damit orientiert sich Effizienz - anders als der ursprünglich häufig synonym verwendete Begriff der Effektivität - zunächst maßgeblich am schonenden Ressourceneinsatz, weniger am Grad der Zielerreichung oder Normerfüllung. ${ }^{31}$

Allerdings können in den anzustellenden Abwägungsvorgang nicht nur Aspekte ökonomischer Effizienz, sondern auch Gesichtspunkte wie organisatorische, soziale, kulturelle oder ökologische Effizienz einbezogen werden. ${ }^{32}$ Für die Verwaltung allgemein und spezifisch für die europäische Mehrebenenverwaltung kann der Effizienzgrundsatz als Rechtsprinzip $^{33}$ und als Handlungs- und Gestaltungsmaßstab ${ }^{34}$ innerhalb der

tisch erscheint hier vor allem die Verwirklichung des rechtsstaatlich wie demokratisch verwurzelten Gebotes der Verantwortungsklarheit, vgl. Schmidt-Aßmann Ordnungsidee (Fn. 6), 309, sowie die Frage nach Kongruenz oder Divergenz der angesprochenen Schichten von Verantwortung.

${ }^{29}$ So W. Hoffmann-Riem Effizienz als Herausforderung an das Verwaltungsrecht Einleitende Problemskizze, in: W. Hoffmann-Riem/E. Schmidt-Aßmann (Hrsg.) Effizienz als Herausforderung an das Verwaltungsrecht, 1998, 11 (17f. mwN); vgl. auch H. H. von Arnim Wirtschaftlichkeit als Rechtsprinzip, 1988, 47; G. Grabatin Effizienz von Organisationen, 1981, 18.

${ }^{30} \mathrm{Zu}$ Begriff und inhaltlichen Anforderungen von Effizienz sowie zu deren Abgrenzung zu Effektivität vgl. Schmidt-Aßmann Ordnungsidee (Fn. 6), 316f. mwN.

31 Zur früheren begrifflichen Gleichsetzung von Effizienz und Effektivität im juristischen Schrifttum vgl. etwa W. Leisner Effizienz als Rechtsprinzip, 1971, 7; P. Häberle Effizienz und Verfassung, AöR 98 (1973), 625 ff.; R. Wahl Verwaltungsverfahren zwischen Verwaltungseffizienz und Rechtsschutzauftrag, VVDStRL 41 (1983), 151 (162f.); ausführlich zur Effektivität als normativ verbindlichem Bestandteil jeder Rechtsordnung M. Hilf/S. Hörmann Effektivität - ein Rechtsprinzip?, FS Tomuschat, $2006 \mathrm{iE}$ wie auch M. Acetto/S. Zleptnig The Principle of Effectiveness: Rethinking Its Role in Community Law, European Public Law 11 (2005), $375 \mathrm{ff}$.

32 So deutlich Röhl Verantwortung (Fn. 27), $1070 \mathrm{mwN}$ in Anlehnung an E. SchmidtAßmann Effizienz als Herausforderung an das Verwaltungsrecht - Perspektiven der verwaltungsrechtlichen Systembildung, in: Hoffmann-Riem/Schmidt-Aßmann (Fn. 29), 245 (246ff. mwN).

${ }^{33}$ In diesem Sinne Hoffmann-Riem Herausforderung (Fn. 29), 19 ff. mwN.

${ }^{34}$ Zur Bedeutung und Wirkung des Effizienzgrundsatzes als Maßstab des Verwaltungshandelns Schmidt-Aßmann Ordnungsidee (Fn. 6), 316ff. mwN. 
vorhandenen rechtlichen Bindungen ${ }^{35}$ insoweit Bedeutung erlangen, als aus ihm die Forderungen nach organisatorischer Effizienz und Verfahrenseffizienz der europäischen Verwaltung folgen. ${ }^{36}$ Organisatorische Effizienz meint einen schonenden Umgang mit organisatorischen Ressourcen, Verfahrenseffizienz eine aufwandbegrenzende Ablauforganisation europäischer Verwaltungsverfahren bei Gewährleistung einer angemessenen Zielerreichung. ${ }^{37}$

Damit spricht Effizienz für eine Verteilung der Verantwortung in der europäischen Mehrebenenverwaltung, die es der Verwaltung ermöglicht, die Verwaltungsaufgaben mit den vorhandenen Verwaltungsressourcen bestmöglich zu erledigen.

\section{Verantwortungsverteilung in der Mehrebenenverwaltung}

Welchen Regeln folgt nun die Verantwortungsverteilung in der Mehrebenenverwaltung, und inwieweit berücksichtigen diese Effizienzgesichtspunkte?

Um diese Fragen beantworten zu können, ist zunächst auf die realen Voraussetzungen für effiziente Verwaltung in Europa und anschließend auf die normativen Vorgaben des Europäischen Verfassungsverbundes, insbesondere dessen Regeln für die Kompetenzverteilung beim Verwaltungsvollzug, einzugehen. ${ }^{38}$

\section{Kooperationsbedürfnis}

Realer Ausgangsbefund für die Verantwortungsverteilung in der europäischen Mehrebenenverwaltung ist ein ausgeprägtes Kooperationsbe-

35 So deutlich Schmidt-Aßmann Ordnungsidee (Fn. 6), 317; Röhl Verantwortung (Fn. 27), $1071 \mathrm{mwN}$; zum Problem bereits deutlich J. Pietzcker Verwaltungsverfahren zwischen Verwaltungseffizienz und Rechtsschutzauftrag, VVDStRL 41 (1983), 193 (196f.).

${ }^{36}$ Auf diese Dimensionen von Effizienz weist bereits Hoffmann-Riem Herausforderung (Fn. 29) hin.

${ }^{37} \mathrm{Zu}$ weiteren Bereichen einer Berücksichtigung der Relation zwischen Aufwand und Nutzen europäisierten Verwaltens etwa H.H. Trute $\S 6$ Die demokratische Legitimation der Verwaltung, in: Hoffmann-Riem/Schmidt-Aßmann/Voßkuhle (Fn. 12), Rn. 116; Röhl Verantwortung (Fn. 27), 1071.

38 Winter (Fn. 5), 255 (259ff.), benennt als gemeinschaftsverfassungsrechtliche Maßstäbe für die Zuordnung von Aufgaben und Organisationsformen die Prinzipien der begrenzten Einzelermächtigung, der Subsidiarität, des institutionellen Gleichgewichts, der Effektivität und des Vorbehalts öffentlicher Verwaltung. 
dürfnis der Verwaltungseinheiten der verschiedenen Ebenen innerhalb und zwischen den Ebenen.

Die Zusammenarbeit der mitgliedstaatlichen Behörden untereinander und mit der Kommission wird als unentbehrliches Instrument für die wirksame und einheitliche Durchsetzung des Gemeinschaftsrechts in der gesamten Gemeinschaft angesehen. ${ }^{39}$ Sie ist erforderlich, weil zentrale Entscheidungen der EG-Verwaltung auf gemeinschaftsweit zuverlässig ermittelter Tatsachengrundlage getroffen und von den nationalen Verwaltungen wirksam und gleichmäßig umgesetzt werden müssen. ${ }^{40}$ Erst Zusammenarbeit ermöglicht die effektive und einheitliche Vollziehung des Gemeinschaftsrechts in allen Mitgliedstaaten trotz divergenter nationaler Verwaltungsrechtsordnungen und Verwaltungstraditionen. ${ }^{41}$ Ebenso ist sie erforderlich als Basis für Vertrauen nationaler Behörden in die ordnungsgemäße Anwendung und Ausführung des Gemeinschaftsrechts durch die Verwaltungen der anderen Mitgliedstaaten, die im Binnenmarkt gemeinschaftsweit wirksame oder von den anderen Mitgliedstaaten anzuerkennende Entscheidungen treffen, etwa Produktzulassungen, Ein- und Ausfuhrbescheinigungen, Zollwertfestsetzungen oder Prüfungsentscheidungen. ${ }^{42}$ Insgesamt kann also die notwendige Ef-

39 So S. Magiera Die Durchsetzung des Gemeinschaftsrechts im europäischen Integrationsprozeß, DÖV 1998, $173(182 \mathrm{mwN})$ spezifisch mit Blick auf binnenmarktbedingte Kontrollbedürfnisse; zu entsprechenden Ansätzen vgl. auch Mitteilung der Kommission an den Rat „Die optimale Gestaltung des Binnenmarkts: Strategisches Programm“, KOM (93) 632 endg. vom 22. 12. 1993, 1 sowie den Bericht der Kommission an den Rat und das Europäische Parlament „Die Entwicklung der Zusammenarbeit der Verwaltungen bei der Anwendung und Durchsetzung des Gemeinschaftsrechts für den Binnenmarkt“, KOM (96) 20 endg. vom 29. 1. 1996; vgl. auch Weiß Schnittstellenprobleme (Fn. 2), 517 (529); Pitschas Europäisches Verwaltungsverfahrensrecht (Fn. 16), 317, geht davon aus, dass Verwaltungskooperation in erster Linie auf den einheitlichen Vollzug des europäischen Gemeinschaftsrechts ausgerichtet ist; Schmidt-Aßmann Strukturen (Fn. 17), 397; zur Zusammenarbeit der mitgliedstaatlichen Verwaltungen und der Kommission bei der Anwendung und Durchsetzung des Gemeinschaftsrechts als Lösungsansatz zur Steigerung der Effizienz des Vollzugs von Gemeinschaftsrecht auch Pühs Vollzug (Fn. 7), $208 \mathrm{ff}$.

40 Weiß Schnittstellenprobleme (Fn. 2), 517 (529 mwN), spricht insoweit davon, dass durch die Kooperation spezifisch europäische Interessen in institutionalisierter Form in den Vollzug eingebracht werden sollen; Vernetzungen der Interessen würden organisatorisch durch Zuständigkeitsvernetzungen abgebildet.

${ }^{41}$ Hierzu Schmidt-Aßmann Ordnungsidee (Fn. 6), 383 sowie Weiß Schnittstellenprobleme (Fn. 2), 517 (529).

42 Auf diese Bedeutung der Verwaltungszusammenarbeit weist anschaulich SchmidtAßmann Ordnungsidee (Fn. 6), 378 mwN hin. 
fektivität und Effizienz des Verwaltungshandelns im Gemeinschaftsraum nur durch Kooperation der Mitgliedstaaten untereinander und mit den EG-Organen gewährleistet werden. ${ }^{43}$

\section{Kooperationskompetenzen}

Damit stellt sich die auf normativer Ebene zu beantwortende Frage, ob die Regeln des europäischen Verfassungsverbundes die tatsächlich erforderliche Verwaltungskooperation zulassen.

\section{a) Kompetenz zur Verwaltung im Verhältnis EG-Mitgliedstaaten}

Die Kompetenzverteilung zwischen Union und Mitgliedstaaten beruht auch hinsichtlich der Verwaltungskompetenzen auf dem in Art. 5 Abs. 1 EGV verorteten Prinzip der begrenzten Einzelermächtigung. ${ }^{44}$ Die Gemeinschaftsverträge weisen den Gemeinschaften keine umfassende Kompetenz zur Administration ihrer eigenen Rechtsordnung $\mathrm{zu},{ }^{45}$ sondern sehen lediglich punktuell begrenzte eigene gemeinschaftliche Vollzugskompetenzen vor. Damit sind nach den Kompetenzvertei-

${ }^{43}$ So auch H.-H. Trute in: H. v. Mangoldt/F. Klein/C. Starck (Hrsg.) GG-Kommentar, Bd. III, 5. Aufl. 2005, Art. 83 Rn. $68 \mathrm{ff}$.

${ }^{44}$ Hierzu ausführlich Streinz EUV/EGV (Fn. 9), Art. 5 Rn. 2, 7 ff. mwN; G. Lienbacher in: J. Schwarze (Hrsg.) EU-Kommentar, Art. 5 EGV Rn. 7 ff. mwN; vgl. auch A. Hatje in: Schwarze, Rn. $43 \mathrm{mwN}$; die Verortung im Grundsatz der verfassungsmäBigen Legalität des Unionshandelns betonen A. von Bogdandy/J. Bast Die vertikale Kompetenzordnung der Europäischen Union. Rechtsdogmatischer Bestand und verfassungsrechtliche Perspektive, EuGRZ 2001, 441 (442f. mwN), die in diesem Zusammenhang auch auf die Bedeutung der Unterscheidung zwischen Verbands- und Organkompetenz hinweisen, dies. ebd., $444 \mathrm{ff}$.; zur Bedeutung des Prinzips für Verwaltung und Verwaltungskompetenzen auch Winter Kompetenzverteilung (Fn. 5), 255 (260 mwN). Aus dem englischsprachigen Schrifttum siehe z.B. T. Hartley The Foundations of European Community Law, 5. Auflage 2003, $105 \mathrm{f}$.

${ }^{45}$ Anders als die Modelle eines dualen Föderalismus (,dual federalism“) mit je eigener Vollzugskompetenz jeder Ebene für das je eigene Recht, vgl. etwa B. Becker Zur föderalen Konstruktion des Vollzugs (Verwaltung) in der Bundesrepublik Deutschland in den Vereinigten Staaten von Amerika und in der Europäischen Gemeinschaft, JöR 39 (1990), 67 (80ff.); eine derartige Zuweisung umfassender Verwaltungskompetenzen kann auch nicht etwa in Art. 211 Spiegelstrich 1 EGV gesehen werden, der vorsieht, dass die Kommission „für die Anwendung dieses Vertrages sowie der von den Organen auf Grund dieses Vertrages geschaffenen Bestimmungen Sorge zu tragen hat", da dies nach Art. 211 Spiegelstrich 3 EGV lediglich nach Maßgabe des Vertrages, also nach Maßgabe der anderweitigen Kompetenzzuweisungen des EGV, geschehen soll, hierzu M. Klepper Vollzugskompetenzen der EG aus abgeleitetem Recht, 2001, 45f. mwN; D. Kugelmann in: Streinz (Fn. 9), Art. 211 Rn. 40 mwN. 
lungsregeln der Gemeinschaftsverfassung grundsätzlich die Mitgliedstaaten zur Verwaltung des Rechts der Europäischen Union berufen. 46

Primärrechtlich vorgesehene Ausnahmen für einen gemeinschaftseigenen, direkten Vollzug durch die Europäische Kommission bestehen vor allem im Bereich der Beihilfenaufsicht ${ }^{47}$ und der öffentlichen Unternehmen. ${ }^{48}$ Daneben sehen die Gemeinschaftsverträge weitere Gemeinschaftsinstitutionen vor, die teilweise Verwaltungsfunktionen wahrnehmen, ${ }^{49}$ so die Europäische Zentralbank, ${ }^{50}$ die Europäische Investitionsbank, ${ }^{51}$ den Europäischen Bürgerbeauftragten ${ }^{52}$ und den Europäischen Datenschutzbeauftragten. ${ }^{53}$ Weitere gemeinschaftliche Vollzugseinrichtungen oder Vollzugsaufgaben sieht das europäische Primärrecht dagegen nicht ausdrücklich vor, ${ }^{54}$ so dass nach Art. 10 EGV in erster Linie die Mitgliedstaaten zum administrativen Vollzug des Gemeinschaftsrechts verpflichtet sind. 55

Allerdings schließen die Gemeinschaftsverträge eine weitergehende Gemeinschaftsverwaltung auch nicht aus. Vielmehr übertragen sie dem Gemeinschaftsgesetzgeber in weitem Umfang die Entscheidung darüber, ob, in welchem Ausmaß und in welchen Formen die EG selbst

\footnotetext{
46 Hierzu etwa $R$. Streinz in: HStR, Bd. VII, § 182 Rn. 4 mwN; EuGH Slg. 1998, I-2661, 2681 f.; Klepper Vollzugskompetenzen (Fn. 45), 21 f. mwN; Pache Interessen der Europäischen Gemeinschaften (Fn. 7), $74 \mathrm{ff}$.

47 Vgl. Art. 88 EGV sowie näher C. Koenig/J. Kühling in: Streinz (Fn. 9), Art. 88 Rn. 1, 15 ff. mwN.

48 Vgl. hierzu Art. 86 Abs. 3 EGV sowie näher C. Jung in C. Callies/M. Ruffert (Hrsg.) Kommentar zu EU-Vertrag und EG-Vertrag 2. Aufl. 2002, Art. 86 Rn. 56 ff.

49 Einen Überblick über die primärrechtlich vorgesehenen Gemeinschaftsorgane und -institutionen gibt etwa Kilb (Fn. 15), 268 (269 mwN).

50 Art. 8 EGV; zu Funktion und Handlungsrahmen U. Palm in: E. Grabitz/M. Hilf (Hrsg.) Das Recht der EU, Art. 8 EGV Rn. 4 ff. mwN.

51 Art. 9, $266 \mathrm{ff}$. EGV sowie M. Rossi in: Callies/Ruffert (Fn. 48), Art. 9 Rn. 4 sowie ders. ebd, Art. 266 Rn. $1 \mathrm{ff}$.

52 Art. 195 EGV.

53 Art. 286 Abs. 2 EGV.

${ }^{54}$ Ebenso A. Hatje Europäische Rechtseinheit durch einheitliche Rechtsdurchsetzung, EuR 1998 Beiheft 1, 8 Fn. 9; zu weiteren Ansätzen für gemeinschaftliche Vollzugskompetenzen im Primärrecht vgl. $R$. von Borries Verwaltungskompetenzen der Europäischen Gemeinschaft, in: O. Due/M. Lutter/J. Schwarze (Hrsg.) Verwaltungskompetenzen der Europäischen Gemeinschaft, FS Everling, Bd. I, 1995, 127 (133ff. mwN).

55 Den Charakter des Art. 10 EGV als Begründung einer Verpflichtung und nicht einer Kompetenz zum Verwaltungsvollzug betont Winter Kompetenzverteilung (Fn. 5), 255 (260) Fn. 16 mwN, der dort in Fn. 17 ebenso zutreffend darauf hinweist, dass auch Art. 211 Spiegelstrich 1 EGV nicht als Übertragung einer allgemeinen Vollzugs- und Aufsichtsgewalt an die Kommission verstanden werden kann.
} 
weitere Verwaltungsaufgaben übernimmt. ${ }^{56}$ Ebenso kann der Gemeinschaftsgesetzgeber in weitem Umfang bestimmen, ob und in welchen Formen eine Kooperation zwischen Gemeinschaft und Mitgliedstaaten oder zwischen den Mitgliedstaaten untereinander beim Vollzug des Gemeinschaftsrechts stattfinden soll. ${ }^{57}$

Kompetentielle Grundlage sowohl der Ausdifferenzierung der EGEigenverwaltung als auch der sekundärrechtlichen Vorschriften, die EG-Eigenverwaltung und Verwaltungskooperation in ihren vielfältigen Erscheinungsformen vorsehen, sind Sachnormen der Verträge, sind funktional ausgerichtete Kompetenzzuweisungen für bestimmte Gemeinschaftspolitiken. ${ }^{58}$

Für die organisatorische Ausdifferenzierung der EG-Eigenverwaltung hat erst jüngst der EuGH entschieden, ${ }^{59}$ dass die Verordnung zur Errichtung der Europäischen Agentur für Netz- und Informationssicherheit 60 zu Recht auf Art. 95 EGV gestützt worden ist. ${ }^{61}$ Damit ist klargestellt, dass selbst so weit gefasste Kompetenzzuweisungen wie diejenige zur

${ }^{56}$ Klepper Vollzugskompetenzen (Fn. 45), $165 \mathrm{f}$. mwN, der in Auseinandersetzung mit dem Grundsatz der institutionellen und verfahrensmäßigen Autonomie der Mitgliedstaaten ausführt: „Der EG-Vertrag macht keine starren Vorgaben für die Verteilung der Verwaltungsaufgaben zwischen Gemeinschaft und Mitgliedstaaten, sondern die Gemeinschaft kann sich aufgrund entsprechender vertraglicher Ermächtigungen weitere Vollzugszuständigkeiten verschaffen“.

57 Sydow Verwaltungskooperation (Fn. 6), 34 ff. mwN, spricht insoweit zusammenfassend von „Kooperationsgesetzgebung“; Priebe Handlungsformen (Fn. 19), 337 (341), weist darauf hin, dass der EGV schon immer „,verwaltungskooperationsfreundlich“ gewesen sei.

58 Ausführlich zur Diskussion um die Kompetenzgrundlagen für Regelungen zur EG-Eigenverwaltung sowie für die sekundärrechtliche Begründung von Kooperationspflichten Weiß Schnittstellenprobleme (Fn. 2), 517 (530ff. mwN); sowie G. Sydow Die Vereinheitlichung des mitgliedstaatlichen Vollzugs des Europarechts in mehrstufigen Verwaltungsverfahren, Die Verwaltung 34 (2001), 517 (530).

59 Nach anfänglichen Zweifeln an der anwendbaren Ermächtigungsgrundlage: In der bisherigen Rechtspraxis ist zunächst Art. 308 EGV als Rechtsgrundlage für die Errichtung von Agenturen angesehen worden, später aus Gründen einer besseren Beteiligung des EP speziellere Rechtsgrundlagen in Anlehnung an die jeweilige Sachmaterie, C. Ohler Rechtmäßige Errichtung der Gemeinschaftsagentur ENISA Anmerkung zu EuGH 2. 5. 2006 - Vereinigtes Königreich Großbritannien und Nordirland/Europäisches Parlament u.a., Rs. C-217/04, EuZW 2006, 373. Aktuell wird die Anwendbarkeit von Art. 308 EGV als Ermächtigungsgrundlage für die Schaffung von unabhängigen Verwaltungseinrichtungen besonders mit Blick auf die Errichtung eines europäischen Finanzmarktregulierers problematisiert, vgl. mwN N. Moloney EC securities regulation, 2002, $883 \mathrm{ff}$.

60 VO (EG) Nr. 460/2004, AB1. EG 2004 Nr. L 77, 1.

${ }^{61}$ EuGH 2. 5. 2006 - Vereinigtes Königreich Großbritannien und Nordirland/Europäisches Parlament u.a., Rs. C-217/04 - EuZW 2006, 369 f. 
Harmonisierung des Binnenmarktes auch die Kompetenz zu verwaltungsorganisatorischen Regelungen und selbst zur Errichtung von eigenen Verwaltungseinrichtungen der EG umfassen können. Voraussetzung ist allein die Notwendigkeit einer solchen Einrichtung für die Verwirklichung des Binnenmarktes, hinsichtlich derer der Gerichtshof den Einschätzungsspielraum des Gemeinschaftsgesetzgebers betont. ${ }^{62}$ Entsprechendes gilt für die Übernahme von Verwaltungsaufgaben durch die EG-Eigenverwaltung ebenso wie für die vielfältigen Formen von Kooperation zwischen mitgliedstaatlichen und europäischen Verwaltungseinrichtungen, die sekundärrechtlich vorgesehen sind. Auch hier besteht eine Gemeinschaftskompetenz aufgrund sachbezogener Kompetenzzuweisungen, ${ }^{63}$ wenn und soweit die Eigenverwaltung oder die Kooperation für die Verwaltung der Sachkompetenz erforderlich ist. 64

Damit gibt es im europäischen Primärrecht keine feste Vorrangregel für die Zuordnung der Vollzugskompetenzen zur europäischen oder mitgliedstaatlichen Ebene. ${ }^{65}$ Vielmehr kann im Rahmen vorhandener Sachkompetenzen der Gemeinschaftsgesetzgeber flexibel über Umfang und Art eigener Vollzugskompetenzen der EG wie über die gemeinschaftsrechtliche Festlegung von Kooperationsverfahren der Mitglied-

62 EuGH 2. 5. 2006 - Vereinigtes Königreich Großbritannien und Nordirland/Europäisches Parlament u.a., Rs. C-217/04 - EuZW 2006, 371 Rn. 44: „Der Gemeinschaftsgesetzgeber kann nämlich auf Grund seiner Sachwürdigung die Schaffung einer Gemeinschaftseinrichtung für notwendig erachten, deren Aufgabe es ist, in Situationen, in denen der Erlass von nicht zwingenden Begleit- und Rahmenmaßnahmen zur Erleichterung der einheitlichen Durchführung und Anwendung von aus Art. 95 EGV gestützten Rechtsakten geeignet erscheint, zur Verwirklichung des Harmonisierungsprozesses beizutragen“; demgegenüber stellt Weiß Schnittstellenprobleme (Fn. 2), 517 (532f. mwN) präziser darauf ab, dass sich der uneinheitliche Vollzug als konkrete Gefährdung der Rechtseinheit im Binnenmarkt erweisen muss.

${ }^{63}$ Einige Kompetenztitel des EGV sehen ausdrücklich die sekundärrechtliche Begründung von Kooperationsstrukturen vor, insbesondere die Kartellbestimmungen des EGV, die Grundlage für den elektronischen Datenaustausch zwischen den Verwaltungen in Art. 156 Abs. 1 i.V.m. Art. 155 EGV oder die Art. 66f. EGV für die Bereiche Visa, Asyl, Einwanderung und freier Personenverkehr sowie Art. 135 EGV im Zollwesen; zu Bedeutung und Grenzen dieser Kompetenzzuweisungen näher Sydow Verwaltungskooperation (Fn. 6), $41 \mathrm{f}$. mwN.

${ }^{64}$ Dies hat der EuGH bereits 1994 bestätigt, vgl. EuGH Slg. 1994, I-3681 (3711f.) zu Art. 9 der damaligen Produktsicherheitsrichtlinie; zur Kritik an dieser Annahme sachgebietsakzessorischer Gemeinschaftskompetenz zur Normierung von Verwaltungskooperation und Übernahme eigener Verwaltungsaufgaben etwa $R$. Wahl/D. Groß Die Europäisierung des Gemeinschaftsrechts, DVB1. 1998, 2 (10ff.); O. Blattern Europäisches Produktzulassungsverfahren, 2003, $32 \mathrm{ff}$.

65 In diesem Sinne auch von Bogdandy/Bast Kompetenzordnung (Fn. 44), 441 (447). 
staaten untereinander oder mit der EG-Eigenverwaltung entscheiden. ${ }^{66}$ Begrenzt werden die entsprechenden Gemeinschaftskompetenzen vor allem durch das Subsidiaritätsprinzip als Kompetenzausübungsrege ${ }^{67}$ sowie den Verhältnismäßigkeitsgrundsatz. ${ }^{68}$

In diesem Rahmen ermöglicht die Gemeinschaftsverfassung eine flexible und sachorientierte Verantwortungszuweisung innerhalb der europäischen Mehrebenenverwaltung, bei der Gesichtspunkte der Effektivität oder Effizienz des Vollzugs der Gemeinschaftsrechtsordnung für die Zuständigkeitsverteilung oder Zuständigkeitsverflechtung berücksichtigt werden können.

\section{b) Kompetenz zur Verwaltung im deutschen Bundesstaat}

Eine vergleichbare Flexibilität ist im deutschen Bundesstaat nicht auszumachen. Hier erfolgt der Vollzug des Gemeinschaftsrechts - wie der des nationalen deutschen Rechts - im Rahmen des durch die Art. 30, $83 \mathrm{ff}$. GG ausgestalteten Exekutivföderalismus grundsätzlich durch die Bundesländer. ${ }^{69}$ Dabei trifft das Grundgesetz keine ausdrückliche Regelung für den Vollzug des Gemeinschaftsrechts, ${ }^{70}$ mit Ausnahme des Art. 108 Abs. 1 S. 1 GG, der vorsieht, dass Abgaben im Rahmen der Europäischen Gemeinschaften durch Bundesfinanzbehörden verwaltet werden. ${ }^{71}$ Die bereits seit langem vorgeschlagene Ergänzung des Grund-

66 Ebenso Schmidt-Aßmann Verfassungsprinzipien (Fn. 12), Rn. 20; zur mangelnden Differenzierung der Kompetenznormen des EGV zwischen legislativen und administrativen Handlungsformen $H$. D. Jarass Die Kompetenzverteilung zwischen der Europäischen Gemeinschaft und den Mitgliedsstaaten, AöR 121 (1996), 173 (181); zusammenfassend auch Sydow Verwaltungskooperation (Fn. 6), $44 \mathrm{mwN}$.

${ }^{67}$ Ausführlich zum Subsidiaritätsprinzip als verwaltungsrelevantem Grundsatz der Aufgabenzuweisung oder Aufgabenwahrnehmung Winter Kompetenzverteilung (Fn. 5), 255 (260ff. mwN), der hier davon ausgeht, dass reine Effizienzgewinne keine hinreichenden Gründe für eine Hochzonung der Aufgabenwahrnehmung darstellen.

68 Zur begrenzenden Funktion dieser Grundsätze instruktiv von Bogdandy/Bast Kompetenzordnung (Fn. 44), 441 (451 mwN); vgl. auch C. Callies, in: ders./Ruffert (Fn. 48), Art. 5 EGV Rn. 6; spezifisch zur Bedeutung des Subsidiaritätsprinzips mit Blick auf horizontale und vertikale Kooperationsformen ausführlich Sydow Verwaltungskooperation (Fn. 6), 46ff. mwN.

${ }^{69}$ Näher zu der unterschiedlichen verfassungsrechtlichen Grundlage dieses Grundsatzes beim mittelbaren und beim unmittelbaren Vollzug von Gemeinschaftsrecht und zur Frage der unmittelbaren oder analogen Anwendung der Art. 83 ff. GG beim unmittelbaren Vollzug Streinz (Fn. 48), Rn. 58f. mwN; ausführlich Suerbaum Kompetenzverteilung (Fn. 7), $207 \mathrm{ff}$.

${ }^{70}$ G. Hermes in: H. Dreier (Hrsg.) Grundgesetz-Kommentar, Bd. 3, 2000, Art. 83 Rn. 7 mwN.

${ }^{71}$ Hierzu näher Suerbaum Kompetenzverteilung (Fn. 7), 211 ff. mwN, dort auch zum in der Praxis wichtigsten Bereich der Gemeinsamen Marktorganisationen und dessen 
gesetzes um eine ausdrückliche Regelung für den Vollzug von europäischem Gemeinschaftsrecht ist auch in der jüngsten Föderalismusreform nicht verwirklicht worden. ${ }^{72}$

Allerdings stellt sich auch beim Vollzug von Gemeinschaftsrecht im deutschen Bundesstaat die Frage nach den Einwirkungsmöglichkeiten des Bundes auf die Vollzugstätigkeit der Länder und nach den möglichen Arten des Zusammenwirkens oder der Verflechtungen beider Ebenen. Diese hängen von der Einordnung des Vollzugs von Gemeinschaftsrecht in die Systematik der Art. 30 und 83 ff. GG ab.

Unproblematisch werden auf den mittelbaren Vollzug von Gemeinschaftsrecht, also den Vollzug nationaler normativer Umsetzungsakte zu umsetzungsbedürftigem Gemeinschaftsrecht, die Verwaltungskompetenzverteilungsregeln des Grundgesetzes direkt angewendet. ${ }^{73}$ Problematisch ist demgegenüber der unmittelbare Vollzug direkt anwendbaren Gemeinschaftsrechts. Für diesen soll nicht die allgemeine Zuständigkeitsregelung des Art. $30 \mathrm{GG}$ einschlägig sein, ${ }^{74}$ bei deren Anwendung keine Einwirkungsmöglichkeiten des Bundes aus den Art. 83 ff. GG bestünden. ${ }^{75}$ Vielmehr werden überwiegend die Art. 83 ff. GG entspre-

Regelung in $\S 31$ Abs. 1 S. 1 Nr. 1 des Gesetzes zur Durchführung der gemeinsamen Marktorganisationen, BGB1. I 1986, 1397; diese Verwaltung der Abgaben im Rahmen der Europäischen Gemeinschaften wird als unmittelbare obligatorische Bundesverwaltung bezeichnet, so B. Pieroth in: Jarass/Pieroth, GG, Art. 108 Rn. 3.

72 Zum Vorschlag der Enquete-Kommission Verfassungsreform aus dem Jahr 1976, das Grundgesetz um einen Art. 90 a zu ergänzen, der wie folgt lauten sollte: „Für die Ausführung von Rechtsvorschriften einer zwischenstaatlichen Einrichtung, auf die der Bund gemäß Artikel 24 Hoheitsrechte übertragen hat, gelten die Regelungen für die Ausführung von Bundesgesetzen entsprechend. Sind die Vorschriften hiernach von den Ländern als eigene Angelegenheit auszuführen, so kann durch Gesetz mit Zustimmung des Bundesrates abweichend hiervon bestimmt werden, dass sie von den Ländern im Auftrag des Bundes ausgeführt werden“, BT-Drs 7/5924, 144, vgl. Suerbaum Kompetenzverteilung (Fn. 7), 211.

73 So weit also die Umsetzung durch Landesrecht erfolgt, besteht eine Verwaltungskompetenz der Länder auf der Grundlage von Art. 30 GG, so weit das Gemeinschaftsrecht durch Bundesgesetz umgesetzt wird, richten sich die Verwaltungskompetenzen nach den Art. 83 ff. GG, näher Suerbaum Kompetenzverteilung (Fn. 7), 221 ff. mwN; Kahl (Fn. 6), 341 (346).

${ }^{74}$ Als Ansatz für die Heranziehung des Art. 30 GG werden gelegentlich die Ausführungen des Bundesverfassungsgerichts im Ersten Rundfunk-Urteil herangezogen, nach denen die Gesetzgebungskompetenzen des Bundes die äußerste Grenze seiner Verwaltungskompetenzen bilden, vgl. in diesem Sinne BVerfGE 12, 205 (229); 15, 1 (16); 78, 374 (386) sowie hierzu mit Blick auf den Vollzug von Gemeinschaftsrecht Suerbaum Kompetenzverteilung (Fn. 7), 227.

75 In der Tat ist bei unmittelbar geltendem Gemeinschaftsrecht für Gesetzgebungskompetenzen des Bundes kein Raum, so deutlich Suerbaum Kompetenzverteilung 
chend auf den Vollzug von Gemeinschaftsrecht angewendet, der Vollzug von Gemeinschaftsrecht also der Ausführung von Bundesgesetzen gleichgestellt. ${ }^{76}$ Damit werden die beim Vollzug innerstaatlichen Rechts für die Bereiche der Bundesgesetzgebung vorgesehenen Aufsichts- und Weisungsrechte des Bundes dem Bund beim Vollzug von Gemeinschaftsrecht unabhängig von der innerstaatlichen Kompetenzverteilung für die Gesetzgebung zuerkannt. ${ }^{77}$ Teilweise wird zur Begrenzung dieser Einwirkungsmöglichkeiten des Bundes auch die Anwendung der Art. 83 ff. GG nach Maßgabe der innerstaatlichen Verteilung der Gesetzgebungs- und Verwaltungskompetenzen vorgeschlagen. ${ }^{78}$

Über seine Aufsichts- und Weisungsrechte hinaus kann der Bund den Vollzug von Gemeinschaftsrecht über seine Kompetenz zur Errichtung selbständiger Bundesoberbehörden aus Art. 87 Abs. 3 GG an sich ziehen. Von dieser vom Grundgesetz an keine besonderen Voraussetzungen zum Schutze des Ländervollzugs geknüpften Möglichkeit hat der Bund weitreichenden Gebrauch gemacht. ${ }^{79}$ Bundesunmittelbare Anstal-

(Fn. 7), 227, so dass bei Betrachtung der Grenzen der Gesetzgebungskompetenzen als äußerste Grenzen auch der Verwaltungskompetenzen eine Bundesverwaltungskompetenz ausgeschlossen erscheint; zu den Gründen für die mangelnde Übertragbarkeit dieser Grenze für Verwaltungskompetenzen auf den Vollzug von Gemeinschaftsrecht mit unterschiedlichen Ansätzen einerseits W. Kössinger Die Durchführung des Europäischen Gemeinschaftsrechts im Bundesstaat, 1989, 53 und anderseits überzeugend Suerbaum Kompetenzverteilung (Fn. 7), $227 \mathrm{ff}$. mwN; Folge der Annahme genereller Verwaltungskompetenzen der Bundesländer auf der Grundlage des Art. 30 GG wäre eine durchaus erhebliche Kompetenzverschiebung vom Bund zu den Ländern beim Vollzug von Gemeinschaftsrecht gegenüber der sonstigen innerstaatlichen Kompetenzverteilung, vgl. hierzu deutlich Hermes (Fn. 70), Art. 83 Rn. 8 mwN; Suerbaum Kompetenzverteilung (Fn. 7), 227; vgl. auch Kössinger Durchführung, 53.

${ }^{76}$ Ausführlich Hermes (Fn. 70), Art. 83 Rn. 9 mwN; vgl. auch Hatje Steuerung (Fn. 7), 51; P. Lerche in: T. Maunz/G. Dürig (Hrsg.) GG, Bd. V, Art. 83 Rn. 51 mwN.

77 So ausführlich Suerbaum Kompetenzverteilung (Fn. 7), 236 und im Anschluss hieran Hermes (Fn. 70), Art. 83 Rn. 9 mwN.

${ }_{78}$ In diesem Sinne Hermes (Fn. 70), Art. 83 Rn. 10 mwN; Suerbaum Kompetenzverteilung (Fn. 7), 237 ff. mwN; Kössinger Durchführung (Fn. 75), 53; Kadelbach Allgemeines Verwaltungsrecht (Fn. 6), 237; D. H. Scheuing Rechtsprobleme bei der Durchsetzung des Gemeinschaftsrechts in der Bundesrepublik Deutschland, EuR 1985, 229 (224); zur spezifischen Situation der konkurrierenden Bundesgesetzgebungskompetenz, bei der bis zum Erlass einer bundesgesetzlichen Regelung die Bundesländer die Kompetenz zur Rechtsetzung besitzen, bei der aber bei entsprechender Behandlung des Vollzugs von Gemeinschaftsrecht nicht das tatsächliche Handeln des Bundesgesetzgebers, das gemeinschaftsrechtlich unzulässig wäre, sondern allein das Vorliegen der Voraussetzungen des Art. 72 Abs. 2 GG für die Anwendung der Art. 83 ff. GG erforderlich ist, näher Suerbaum Kompetenzverteilung (Fn. 7), 242 ff. mwN.

${ }^{79} \mathrm{Zu}$ dieser Möglichkeit der Ausdehnung der Bundesverwaltungszuständigkeiten Suerbaum Kompetenzverteilung (Fn. 7), 230f. mwN; dort auch näher zur Bundesanstalt 
ten für den Vollzug von Europarecht sind insbesondere bei der Verwaltung der landwirtschaftlichen Marktordnungen eingerichtet worden, 80 und zahlreiche Verwaltungsfunktionen insbesondere in Kooperationsstrukturen sind auf bestehende Bundesoberbehörden und bundesunmittelbare Anstalten übertragen worden. ${ }^{81}$

\section{c) Kooperationsverbote}

Wie verhalten sich damit europäisches Primärrecht und Grundgesetz zu der für einen effektiven Vollzug des Gemeinschaftsrechts erforderlichen Verwaltungszusammenarbeit im Mehrebenensystem?

Weder die europäische Verfassung noch das Grundgesetz kennen grundsätzliche Kooperationsverbote. Wie dargelegt, lässt die europäische Verfassung die flexible Ausgestaltung der Verantwortungsverteilung zwischen EU und Mitgliedstaaten in Gestalt von Kooperationsverfahren und Kooperationsinstitutionen ohne weitere Einschränkung zu. Nach dem Grundgesetz ist Verwaltungszusammenarbeit dagegen an strengere Voraussetzungen gebunden: Das Grundgesetz stellt zwar kein rigides Verbot der Mischverwaltung auf, ${ }^{82}$ aber es begrenzt die Möglichkeiten des Verwaltungsvollzugs grundsätzlich auf die im Grundgesetz vorgesehenen Formen. ${ }^{83}$ Diese Begrenzung soll die Kompetenzvertei-

für Landwirtschaft und Ernährung als der zentral für den Vollzug der Gemeinsamen Marktordnungen zuständigen Behörde; vgl. auch M. Burgi in: Mangoldt/ Klein/Starck (Fn. 43), Art. 87 Abs. 3 Rn. 93; Hermes (Fn. 70), Art. 83 Rn. 67.

80 So die frühere Bundesanstalt für landwirtschaftliche Marktordnung, die allerdings zum 1. 1. 1995 mit dem Bundesamt für Ernährung und Forstwirtschaft zur Bundesanstalt für Landwirtschaft und Ernährung zusammengeführt worden ist, die zwar immer noch weit überwiegend, aber nicht mehr ausschließlich Europarecht vollzieht, vgl. hierzu Gesetz über die Errichtung einer Bundesanstalt für Landwirtschaft und Ernährung vom 2. 8. 1994, BGB1. I, 2018.

${ }^{81}$ Diese Vorgehensweise liegt nahe, wenn in gemeinschaftsrechtlich vorgegebenen Kooperationsstrukturen eine einzige nationale Behörde als Kooperationspartner zu benennen ist, vgl. ausführlicher Sydow Verwaltungskooperation (Fn. 6), $77 \mathrm{f}$. mwN.

82 Terminologie auch bei BVerfGE 32, 145 (156); 39, 96 (120); 41, 291 (311); hierzu ausführlich H. H. Klein Verwaltungskompetenzen von Bund und Ländern in der Rechtsprechung des Bundesverfassungsgerichts, in: FG Bundesverfassungsgericht, Bd. II, 1976, 278 (286ff.); K. Stern Staatsrecht, Bd. II, 1980, 832; Lerche(Fn. 76), Art. 83 Rn. 85 mwN.

${ }^{83}$ Ungeachtet der Rede vom sogenannten Verbot der Mischverwaltung sieht das Grundgesetz durchaus verschiedene Formen einer „Mischverwaltung“ im deskriptiven Sinne vor: etwa die Bundesauftragsverwaltung kann aufgrund der weitreichenden Abhängigkeit der Länder von Vorgaben des Bundes ebenso als Mischverwaltung bezeichnet werden wie der Behördenaufbau in der Finanzverwaltung oder die Gemeinschaftsaufgaben nach Art. 91a, 91b GG. 
lung zwischen Bund und Ländern sichern ${ }^{84}$ und Verantwortungsklarheit gewährleisten. ${ }^{85}$ Daher soll im Grundsatz das System der Verwaltungstypen des Grundgesetzes nicht durch neue, nicht im Grundgesetz vorgesehene Verwaltungs- oder Kombinationsformen überspielt werden. ${ }^{86}$

Voraussetzung für Verwaltungszusammenarbeit nach dem Grundgesetz ist also, dass diese im Grundgesetz vorgesehen oder angelegt ist. Diese Voraussetzung ist etwa bei den grundgesetzlich vorgesehenen Kooperationsmöglichkeiten zwischen Bund und Ländern im Rahmen der Bundesaufsicht oder bei der Finanzverwaltung auch in für den Vollzug von Gemeinschaftsrecht relevanten Bereichen gegeben, die flexible Kreation neuer weiterer Formen der Zusammenarbeit lässt das Grundgesetz dagegen nicht zu.

\section{Formen kooperativer Verantwortungsverteilung in der europäischen Mehrebenenverwaltung}

Die mehrebenentypische Verflechtung von Verwaltungsverantwortlichkeiten findet sich aufgrund der festgestellten flexibleren Kompetenzverteilung in erster Linie zwischen den beiden oberen Ebenen, zwischen der EU und ihren Mitgliedstaaten. Auf der Grundlage entsprechender Kooperationsgesetzgebung des Gemeinschaftsgesetzgebers sind vielfältige Formen europäischer Verwaltungskooperation entstanden, die in unterschiedlichen Variationen und Mischformen die nationalen Verwaltungen untereinander und mit der europäischen Verwaltung verschränken und sie in unterschiedliche Strukturen des verbundenen Vollzugs von Gemeinschaftsrecht mit variabler Aufgaben- und Befugnisverteilung einbinden. ${ }^{87}$

Unterschiedliche Systematisierungen sind möglich. Kooperationsvorgänge finden ihre Grundlage im Primärrecht oder im Sekundärrecht der EG ${ }^{88}$ In Betracht kommt eine Differenzierung nach Kooperation in In-

${ }^{84}$ Ausführlich Hermes (Fn. 70), Art. 83 Rn. 47 ff. mwN.

85 Deutlich in diesem Sinne aus der neueren Rechtsprechung BVerfGE 108, 169 (178f., 181f.).

${ }^{86}$ BVerfGE 13, 271 (275f.); Lerche(Fn. 82) Art. 83 Rn. 84 ff.; Hermes (Fn. 70), Art. 83 Rn. $51 \mathrm{mwN}$.

${ }^{87}$ In diesem Sinne auch Gundel Verwaltung (Fn. 5), Rn. 3 ff. mwN; Priebe Handlungsformen (Fn. 19), 337 (339), betont: „Es gibt kein einheitliches Modell für Verwaltungskooperation in Europa“.

${ }^{88}$ Mit diesem Ansatz etwa Gundel Verwaltung (Fn. 5), Rn. 119 ff. mwN, der als primärrechtliche Kooperationsinstrumente lediglich das sehr beschränkt einsetzbare Vertragsverletzungsverfahren sowie die in Einzelbereichen bestehenden primärrechtlichen 
formations- und Kommunikationsverbänden ${ }^{89}$ und der Mitwirkung in vertikalen und horizontalen Entscheidungszusammenhängen. ${ }^{90}$ Es lassen sich Informationsnetzwerke, ${ }^{91}$ die Einbindung von Ausschüssen in Entscheidungsabläufe ${ }^{92}$ und die verschiedenen Formen der Gemeinschaftsaufsicht trennen. ${ }^{93}$ Auch eine Differenzierung zwischen zentralen

Entscheidungsbefugnisse der Kommission anspricht; sehr viel mannigfaltiger fallen entsprechend die Bereiche sekundärrechtlicher Kooperationsinstrumente aus, bei denen etwa die Anlastung, Inspektionen und Kontrollen, Genehmigungsvorbehalte für normative Maßnahmen der Mitgliedstaaten, gestufte Entscheidungsverfahren und Regulierungsnetzwerke mit Letztentscheidungsrecht der Kommission unterschieden werden; einen weiteren großen Bereich der Mehrebenenverwaltung macht danach die horizontale Kooperation der nationalen Behörden aus, vgl. ders. ebd., Rn. $145 \mathrm{ff}$. mwN.

${ }^{89}$ Diese wird als Grundelement jeder Verwaltungskooperation angesehen: „Mindestinhalt jeder Kooperation ist die Information“, so Sommer Verwaltungskooperation (Fn. 19), 74f. mwN in Anlehnung an Schmidt-Aßmann Verwaltungskooperation (Fn. 10), 270 (290), der dort auch darauf hinweist, dass Kommunikation gleichzeitig die weitere Bereitschaft zur Kooperation erhöhe, dies. ebd.

${ }^{90}$ So Schmidt-Aßmann Verwaltungskooperation (Fn. 10), 270 (275ff. mwN), dort auch mit weiteren Differenzierungsansätzen wie horizontaler und vertikaler Kooperation, bi-, tri- und multilateral angelegter Kooperation, punktueller und dauerhafter Kooperation, Kooperation mit und ohne Durchgriffswirkung und Kooperation zur Schaffung einfacher und komplexer Entscheidungszusammenhänge.

${ }^{91}$ Diese können durch hochintegrierte Informationsagenturen wie die Europäische Umweltagentur in Kopenhagen oder die Beobachtungsstelle für Drogen in Lissabon oder durch horizontal ausgerichtete Informationsverbünde konstituiert werden, vgl. systematisch zu dieser Differenzierung $A$. v. Bogdandy Information und Kommunikation in der Europäischen Union: föderale Strukturen im supranationalem Umfeld, in: E. Schmidt-Aßmann/W. Hoffmann-Riem (Hrsg.) Verwaltungsrecht in der Informationsgesellschaft, 2000, 133 (172ff.); zu den Informationsagenturen D. Fischer-Appelt Agenturen der Europäischen Gemeinschaft, 1999, 55 ff. Beispiele für Informationsverbünde bilden etwa das Zollinformationssystem ZIS oder das Verwaltungs- und Kontrollsystem INVEKOS im Agrarbereich, hierzu Schmidt-Aßmann Verwaltungsverbund (Fn. 17), 16f. mwN.

${ }^{92} \mathrm{Zu}$ unterscheiden sind hier vor allem die primär dem vertikalen Interessenausgleich mit Vertretern der mitgliedstaatlichen Regierungen dienenden Komitologieausschüsse von den auf die Einbindung externen Sachverstandes in die Kommissionsentscheidungen ausgerichteten wissenschaftlichen Ausschüssen, hierzu Schmidt-Aßmann Verwaltungsverbund (Fn. 17), 17 ff. mwN.

${ }_{93}$ Die Kommission besitzt gegenüber den mitgliedstaatlichen Verwaltungen kein allgemeines Auskunfts- oder Weisungsrecht, vgl. nur Gundel Verwaltung (Fn. 5), Rn. 119 mwN; demgegenüber haben sich differenzierte Handlungsformen faktischer Aufsicht und Kontrolle herausgebildet, die sich etwa an der Beihilfeaufsicht der Art. $87 \mathrm{ff}$. EGV, dem Rechnungsabschluss im Agrarrecht, den in einzelnen Rechtsgebieten vorgesehenen Inspektionen und der Installation reflexiver Kontrollstrukturen orientieren, hierzu zusammenfassend Schmidt-Aßmann Verwaltungsverbund (Fn. 17), $20 \mathrm{ff} . \mathrm{mwN}$; zum Rechnungsabschluss $R$. Mögele Die Behandlung fehlerhafter Ausgaben im Finanzie- 
und dezentralen Ansätzen zur Steigerung der Effizienz des Verwaltungsvollzuges $^{94}$ liegt nicht fern.

Um die unterschiedlichen Kooperationsformen im Rahmen der grundsätzlichen Kompetenzverteilung zwischen EG und Mitgliedstaaten zu erfassen, sollen nachfolgend Kooperationselemente im Rahmen des Einzelvollzugs, des Vollzugs mit Referenzentscheidung, des transnationalen Vollzugs und des Direktvollzugs von Gemeinschaftsrecht ${ }^{95}$ sowie bereichsübergreifend Formen der institutionalisierten Kooperation angesprochen werden.

\section{Einzelvollzug}

Beim mitgliedstaatlichen Einzelvollzug bestehen seit Gründung der EG Aufsichtsrechte der Kommission aus ihrer Rolle als Hüterin der Verträge, zu deren Wahrnehmung teilweise Notifikationspflichten der Mitgliedstaaten ${ }^{96}$ oder allgemeine Berichtspflichten ${ }^{97}$ vorgesehen sind.

rungssystem der gemeinsamen Agrarpolitik, 1997, $209 \mathrm{ff}$; zu Inspektionen A. David Inspektionen im Europäischen Verwaltungsrecht, 2003, $30 \mathrm{ff}$. mwN.

94 So die Einteilung bei Pühs Vollzug (Fn. 7), 206 ff. mwN; vgl. auch Huber Kooperationsverhältnis (Fn. 18), 128, der zwischen den Kooperationsformen des indirekten Vollzugs durch nationale Behörden unter Aufsicht der Kommission, des indirekten Vollzugs durch nationale Behörden unter notwendiger Mitwirkung der Kommission, der Mitwirkung nationaler Behörden beim Erlass von Durchführungsvorschriften durch die Kommission sowie dem direkten Vollzug des Unionsrechts durch die Kommission oder sonstige Stellen oder Einrichtungen der EU/EG, bei dem die nationalen Behörden zur Amts- oder Vollzugshilfe verpflichtet sind, unterscheidet.

95 Zur Systembildung im europäischen Kooperationsrecht und zu den angesprochenen Vollzugsmodellen Sydow Verwaltungskooperation (Fn. 6), $118 \mathrm{ff}$. mwN; die Modellbildung erfolgt bei Sydow im Referenzgebiet des Produktzulassungsrechts, in dem sich unterschiedliche horizontale wie vertikale Kooperationsformen ergänzen und dem daher eine weitgehende Eignung zur Verallgemeinerung bezüglich Möglichkeiten und Formen der Verwaltungskooperation nicht abzusprechen ist; zu ergänzen ist die Vierteilung der Vollzugsmodelle um die Spezifika der Kooperation im weiteren Bereich der so genannten Ausdifferenzierung der EG-Eigenverwaltung, hierzu etwa jüngst G. Sydow Externalisierung und institutionelle Ausdifferenzierung, VerwArch 97 (2006), $1 \mathrm{ff} . \mathrm{mwN}$; ähnliche, aber mit etwas anderem Ansatz erfolgende Systematisierung bei Schmidt-A $\beta$ mann Strukturen (Fn. 17), 397 ff. mwN, der die Kooperationsformen in schlichte Handlungszusammenhänge, vertikal organisierte Entscheidungszusammenhänge, horizontal organisierte Entscheidungszusammenhänge und Formen institutionalisierter Kooperation untergliedert.

96 Etwa bei Ergreifung von Schutzmaßnahmen im Bereich des Umweltrechts: hier sind Notifikationspflichten primärrechtlich vorgesehen in Art. 176 S. 3 EGV, hierzu W. Kahl in: Streinz (Fn. 9), Art. 176 Rn. 15 ff.

97 Beispiele bei Huber Kooperationsverhältnis (Fn. 18), 129 mwN in Fn. 13. 
Ergänzend haben sich - ungeachtet des Fehlens rechtlich bindender Weisungsbefugnisse - Formen der rechtlich nicht bindenden, aber faktisch effektiven Steuerung des Verhaltens der mitgliedstaatlichen Verwaltungen durch die Europäische Kommission entwickelt, etwa durch Informationsvermerke ${ }^{98}$ oder das Rechnungsabschlussverfahren. ${ }^{99}$ Unmittelbare Weisungs-, Inspektions- und Kontrollbefugnisse bestehen auf sekundärrechtlicher Grundlage. 100

Deutlich gesteigert ist die Intensität der Verantwortungsverflechtung beim nationalen Einzelvollzug im Rahmen sogenannter gestufter Verwaltungsverfahren, innerhalb derer nationale Entscheidungen vertikal mit Anhörungs-, Zustimmungs-, Genehmigungs- und Derogationsrechten der Kommission in vielfältiger Weise verkoppelt werden. ${ }^{101} \mathrm{Hier}$ sind regelmäßig102 die nationalen Behörden im Außenverhältnis gegenüber dem Bürger für den Vollzug des Gemeinschaftsrechts verantwortlich, innerhalb der Mehrebenenverwaltung ist jedoch ihre Entscheidungsbefugnis an die Mitwirkung der Kommission im Innenverhältnis gebunden. ${ }^{103}$

Letztlich können die Kooperationsbeziehungen ein solches Maß an Enge und Verdichtung erreichen, dass bereits von einer gemeinsamen oder geteilten Verwaltung die Rede ist, wie dies insbesondere für die Verwaltung der europäischen Strukturfonds angenommen wird. ${ }^{104}$

$98 \mathrm{Zu}$ Rechtsnatur und Wirkung der Informationsvermerke etwa Pache Interessen der Europäischen Gemeinschaften (Fn. 7), 286f. mwN.

${ }^{99}$ Hierzu G. Thiele in: Callies/Ruffert (Fn. 48), Art. 34 Rn. 48 ff. mwN; Pühs Vollzug (Fn. 7), 238f.

100 Beispiele etwa bei Huber Kooperationsverhältnis (Fn. 18), 129f. mwN, dort auch zur besonderen ergänzenden Bedeutung informaler Implementationskontrolle durch Europäisches Parlament und Öffentlichkeit für den mitgliedstaatlichen Vollzug.

101 Ausführlich zu Modellen gestufter Verwaltungsverfahren und deren Entscheidungsstrukturen sowie zu unterschiedlichen Ausgestaltungsoptionen Nehl Europäisches Verwaltungsverfahren (Fn. 7), 39 ff. mwN; Huber Kooperationsverhältnis (Fn. 18), 130 f. mwN; ders. Recht der Europäischen Integration, 2. Auflage 2002, § 24 Rn. 12; Schmidt-Aßmann Strukturen (Fn. 17), 399.

${ }^{102} \mathrm{Zu}$ Abweichungsmöglichkeiten je nach Ausgestaltung des konkreten Verfahrens mehrstufiger Entscheidung Nehl Europäisches Verwaltungsverfahren (Fn. 7), 39.

103 Beispiele sind etwa die Beihilfenaufsicht oder Abweichungsrechte im Binnenmarkt, vgl. Huber Kooperationsverhältnis (Fn. 18), 130 ff. mwN sowie das Erstattungsverfahren im Zollrecht oder die Rückforderung irregulär ausgezahlter Strukturfondsmittel, so Schmidt-Aßmann Strukturen (Fn. 17), $399 \mathrm{mwN}$; ausführlich zum Einzelvollzugsmodell am Beispiel des Arzneimittelzulassungsrechts und seiner Entwicklung Sydow Verwaltungskooperation (Fn. 6), $126 \mathrm{ff}$. mwN.

104 Ausführlich hierzu B. Schöndorf-Haubold Die Strukturfonds der Europäischen Gemeinschaft, 2005 sowie ders. Gemeinsame Europäische Verwaltung, in: Schmidt-Aßmann/Schöndorf-Haubold (Fn. 17), 26 ff. mwN, zu den Besonderheiten gemeinsamer 


\section{Referenzentscheidung}

In zahlreichen Rechtsgebieten ursprünglichen Einzelvollzugs hat sich dieser zwischenzeitlich zum Vollzug durch Referenzentscheidung gewandelt, ${ }^{105}$ der auch als Verfahren der gegenseitigen Anerkennung bezeichnet wird ${ }^{106}$ und insbesondere im Produktzulassungsrecht verbreitet ist.107 Der Verwaltungsvollzug erfolgt hier weiterhin einzelstaatlich, ${ }^{108}$ die anderen Mitgliedstaaten sind jedoch kraft Gemeinschaftsrechts verpflichtet, die mitgliedstaatliche Entscheidung anzuerkennen, hinsichtlich derer ihnen nur begrenzte eigene Überprüfungsbefugnisse zustehen. ${ }^{109}$

Verweigern andere Mitgliedstaaten die Anerkennung, ist regelmäßig ein mehrstufig ausgestaltetes Divergenzbereinigungsverfahren vorgesehen, in dem die Entscheidungskompetenz auf die Kommission übergehen kann. ${ }^{110}$

\section{Transnationalität}

Bei der Wirkung einer im nationalen Einzelvollzug getroffenen Entscheidung noch weiter geht das sogenannte Transnationalitätsmodell, bei dem Entscheidungen nationaler Behörden durch EG-Recht unmittelbar, ohne zusätzlichen Anerkennungs- oder Transformationsakt, gemeinschaftsweite Wirkung beigemessen wird. ${ }^{111}$ Einer solchen trans-

Verwaltung ebd., $54 \mathrm{ff}$; von geteilter Verwaltung spricht Trute Legitimation (Fn. 37), Rn. 110, und sieht diese wegen der besonders intensiven Kooperation in vertikaler Richtung als eigenen Verwaltungstyp an. Ausführlich zu diesem Problemkreis auch Bernard Multilevel Governance (Fn. 2), $99 \mathrm{ff}$.

105 Diesen Wandel zeigt exemplarisch für das Arzneimittelzulassungsrecht Sydow Verwaltungskooperation (Fn. 6), $129 \mathrm{ff}$. mwN auf.

106 Zum Referenzentscheidungsmodell näher Sydow Verwaltungskooperation (Fn. 6), $181 \mathrm{ff}$. mwN; üblich ist teilweise auch eine Bezeichnung als Transnationalität unter Prüfungsvorbehalt.

107 So etwa Schmidt-Aßmann Strukturen (Fn. 17), 400 mwN.

108 Sydow Verwaltungskooperation (Fn. 6), $182 \mathrm{ff}$. mwN, insbesondere müssen im nationalen Verwaltungsverfahren nicht zwingend Elemente vertikaler oder horizontaler Kooperation vorhanden sein.

109 Schmidt-Aßmann Strukturen (Fn. 17), 400; zu den sonstigen Wirkungen der Durchführung des nationalen Referenzentscheidungsverfahrens, insbesondere der entweder an der Verfahrenseinleitung oder an der Verfahrensbeendigung anknüpfenden Sperrwirkung des Referenzverfahrens gegenüber parallelen Verfahren anderer Mitgliedstaaten Sydow Verwaltungskooperation (Fn. 6), 183 ff. mwN.

110 Zusammenfassend Sydow Verwaltungskooperation (Fn. 6), $188 \mathrm{ff}$. mwN; SchmidtAßmann Strukturen (Fn. 17), 400.

111 Zur Transnationalität als Form europäischen Verwaltens näher Sydow Verwaltungskooperation (Fn. 6), $138 \mathrm{ff}$. mwN; vgl. auch Gundel Verwaltung (Fn. 5), Rn. $160 \mathrm{ff}$. 
national wirkenden Entscheidung können Kooperationsverfahren mit unterschiedlich starken Beteiligungsrechten der anderen Mitgliedstaaten - von bloßen Anhörungsrechten bis zu Vetopositionen oder Einvernehmensregelungen - vorgelagert sein. Entstehen in diesen Kooperationsverfahren nicht auflösbare Meinungsverschiedenheiten, wird ähnlich wie bei der Referenzentscheidung ein Divergenzbereinigungsverfahren mit abschließender Entscheidungsbefugnis der Kommission durchgeführt. 112

\section{Direktvollzug}

Beim Direktvollzug schließlich erfolgt die Anwendung des Gemeinschaftsrechts bis zur verfahrensabschließenden Entscheidung im AuBenverhältnis unmittelbar durch die EG-Kommission oder andere Teile der sich ausdifferenzierenden EG-Eigenverwaltung. ${ }^{113}$ Diese können insbesondere bei der Entscheidungsvorbereitung oder bei der Implementationskontrolle ${ }^{114}$ mit den mitgliedstaatlichen Verwaltungen kooperieren, indem sie sie etwa für Ermittlungen und Kontrollen vor Ort oder

mwN, dort zu den sekundärrechtlichen Grundlagen dieser transnationalen Wirkung in Rn. $163 \mathrm{ff}$. und zu den Anwendungsbereichen in Rn. $167 \mathrm{ff}$., jeweils mwN; zum transnationalen Verwaltungsakt, der allerdings nur eine spezielle Form transnationalen Handelns in der EU darstellt, seiner Typologie und seinen Wirkungen ausführlich M. Ruffert Der transnationale Verwaltungsakt, Die Verwaltung 34 (2001), 453 ff. mwN; vgl. auch V. Nessler Der transnationale Verwaltungsakt - Zur Dogmatik eines neuen Rechtsinstituts, NVwZ 1995, 863 ff.; zum sogenannten mehrstufig gemeinschaftlichen Verwaltungsakt und einer Abgrenzung hiervon zum transnationalen Verwaltungsakt siehe J. Caspar Zur Vergemeinschaftung von Verwaltungsverfahren am Beispiel von Gentechnik- und reformiertem Lebensmittelrecht, DVB1. 2002, $1437 \mathrm{ff}$.

112 Die Grundstruktur des Zustandekommens eines transnationalen Verwaltungsakts nach Maßgabe der gemeinschaftsrechtlichen Vorgaben erläutert Sydow Verwaltungskooperation (Fn. 6), $150 \mathrm{ff}$. mwN. Treten nach Erlass einer transnational wirkenden Entscheidung Zweifel an deren Rechtmäßigkeit auf, so ist allein der ursprünglich entscheidende Mitgliedstaat zur Aufhebung befugt. Den anderen Mitgliedstaaten stehen aber auf der Grundlage so genannter Schutzklauseln vorläufige Suspensionsbefugnisse zu, durch deren Ausübung ein Kooperationsverfahren zur gemeinschaftsweiten Aufhebung der Ursprungsentscheidung eingeleitet werden kann, vgl. S. Michaels Anerkennungspflichten im Wirtschaftsverwaltungsrecht der Bundesrepublik Deutschland, 2004, $371 \mathrm{ff}$., der insoweit eher einschränkend von Notkompetenzen spricht.

113 Gundel Verwaltung (Fn. 5), Rn. 91 ff.; Sydow Verwaltungskooperation (Fn. 6), $216 \mathrm{f}$. mwN.

${ }^{114}$ Insbesondere hier erfordert die nur sehr begrenzte Kapazität der EU-Eigenverwaltung an eigenen Verwaltungskompetenzen die Kooperation mit den mitgliedstaatlichen Verwaltungen und des Rückgriffs auf diese quasi als verlängerter Arm der EU-Eigenadministration, vgl. Gundel Verwaltung (Fn. 5), Rn. 93. 
zur Vornahme wissenschaftlicher oder technischer Untersuchungen und Prüfungen einsetzen.

Darüber hinaus erfolgt beim Direktvollzug eine Verschränkung der europäischen und der mitgliedstaatlichen Ebene über die Komitologieverfahren, innerhalb derer eine Rückkopplung der gemeinschaftlichen Durchführungsmaßnahmen an die in den Ausschüssen tagenden Regierungsvertreter der Mitgliedstaaten erfolgt. ${ }^{115}$ Auch beim direkten Vollzug des Gemeinschaftsrechts sind vorläufige Suspensionsbefugnisse der Mitgliedstaaten und eine anschließende Kommissionsentscheidung vorgesehen. ${ }^{116}$

\section{Netzwerkbildung und Institutionalisierte Kooperation}

Ergänzt werden diese vollzugstypabhängigen Zusammenarbeitsformen durch die alle Vollzugsvarianten des Gemeinschaftsrechts erfassenden Kooperationsformen der Netzwerkbildung und der institutionalisierten Kooperation, ${ }^{117}$ also das Zusammenwirken der Gemeinschaftsverwaltung und der nationalen Verwaltungen in Kooperationsinstitutionen.

Netzwerkbildung als Bauform der Mehrebenenverwaltung ist dabei eine Organisationsform der Verwaltungszusammenarbeit, bei der selbständig bleibende unterschiedliche Verwaltungseinheiten ${ }^{118}$ in relativ dauerhafte und verdichtete Formen der Zusammenarbeit eingebunden werden, die mittlerweile häufig durch elektronische Vernetzung unterstützt werden.119 Solche Netzwerke haben als Informations- und Kommunikationsnetzwerke von Anfang an die europäische Mehrebenenverwaltung geprägt, mittlerweile erfahren sie auch als verrechtlichte Handlungszusammenhänge zunehmende Anerkennung. ${ }^{120}$ Bedeutsam

115 Instruktiv zu diesem Problemkreis Bernard Multilevel Governance (Fn. 2), $149 \mathrm{ff}$;; vgl. auch $C$. Mensching Der neue Komitologie-Beschluss des Rates, EuZW 2000, $268 \mathrm{ff}$.

116 Nachweise bei Sydow Verwaltungskooperation (Fn. 6), 222.

117 So die zusammenfassende Bezeichnung bei Schmidt-Aßmann Strukturen (Fn. 17), 401.

118 Im Kontext der Verwaltungskooperation werden abweichend vom allgemeinen verwaltungsorganisatorischen Sprachgebrauch als Netzwerke allein Behördennetzwerke verstanden, ebenso Schmidt-Aßmann Verfassungsprinzipien (Fn. 12), Rn. 26 mwN.

119 Näher zum Netzwerkbegriff und zu Konstruktionselementen und Funktionen Sydow Verwaltungskooperation (Fn. 6), 78f. mwN.

${ }^{120} \mathrm{Zu}$ Netzwerken als allgemeiner Form der Zusammenarbeit der Mitgliedstaaten in den verschiedenen Bereichen des Gemeinschaftsrechts etwa Schulze-Fielitz Verfassungsgefüge (Fn. 13), 124f. mwN. 
sind etwa aus jüngerer Zeit das Netzwerk zwischen der EU-Kommission und den nationalen Kartellbehörden zum Vollzug der Kartellverfahrensverordnung ${ }^{121}$ oder der europäische Regulierungsverbund nach den neuen Telekommunikationsrichtlinien. ${ }^{122}$

Teilweise sind Netzwerkstrukturen in der europäischen Mehrebenenverwaltung so weit ausgeformt und verfestigt, dass von institutionalisierter Kooperation gesprochen werden kann. Dies gilt bereits seit langem für die unterschiedlichen Ausschüsse, ${ }^{123}$ insbesondere die etwa 250 zentral mit Vertretern der Mitgliedstaaten besetzten Komitologieausschüsse, ${ }^{124}$ und zunehmend für die Europäischen Agenturen und Ämter. ${ }^{125}$

Die Komitologieausschüsse stellen einen zentralen Kooperationsmechanismus zwischen der Kommission und den nationalstaatlichen

121 VO 1/2003 zur Durchführung der in den Artikeln 81 und 82 des Vertrags niedergelegten Wettbewerbsregeln vom 16. Dezember 2003, AB1. EG 2003 Nr. L 1, 1, vgl. hierzu A. Klees Europäisches Kartellverfahrensrecht, 2005, § 7 Rn. $63 \mathrm{ff}$.; U. Böge/ A. Scheidgen Das neue Netzwerk der Wettbewerbsbehörden in der Europäischen Union, EWS 2002, 201 ff.; H. Jochum Das Bundeskartellamt auf dem Weg nach Europa, VerwArch 94 (2003), 512 (527ff.); A. Fuchs Kontrollierte Dezentralisierung der europäischen Wettbewerbsaufsicht, EuR 2005 Beiheft 2, $77 \mathrm{ff}$.; vergleiche hierzu aus dem englischsprachigen Schrifttum etwa $J$. Venit Brave New World: The Modernization and Decentralization of Enforcement under Articles 81 and 82 of the EC Treaty, CMLRev. 40 (2003), $545 \mathrm{ff}$; A. Riley EC Antitrust Modernisation: The Commission Does Very Nicely - Thank You! Part Two: Between the Idea and the Reality: Decentralisation under Regulation 1, ECLR 24 (2003), 657 ff.; S. Kingston A „new division of responsibilities" in the proposed regulation to modernise the rules implementing Art. 81 and 82 E.C.? A warning call, ECLR 22 (2001), $340 \mathrm{ff}$.

122 Dazu H.-H. Trute Der europäische Regulierungsverbund in der Telekommunikation - ein neues Modell europäischer Verwaltung, in: FS Peter Selmer, 2004, 565 (570ff.); Ladeur/Möllers Regulierungsverbund (Fn. 18), $525 \mathrm{ff}$.

123 Unterschieden werden können Sachverständigengruppen, beratende Ausschüsse und wissenschaftliche Ausschüsse, die von der Kommission selbst zur Erschließung von Sachverstand und Beratung eingesetzt werden, von den durch Ratsbeschluss eingesetzten Komitologieausschüssen, vgl. Sydow Verwaltungskooperation (Fn. 6), 80 mwN, der in Fn. 90 mwN auf die Vielfalt und Heterogenität der Ausschüsse hinweist.

${ }^{124} \mathrm{Zu}$ Grundlagen, Entwicklung und Funktionen nur W. Hummer/W. Obwexer in: Streinz (Fn. 9), Art. 202 Rn. 26 ff. mwN; M. Schweitzer in: Grabitz/Hilf (Fn. 50), Art. 202 Rn. $27 \mathrm{ff}$. mwN.

125 Für die Einordnung dieser Institutionen in den Netzwerkzusammenhang SchulzeFielitz Verfassungsgefüge (Fn. 13), 124f.; zur Dezentralisierung der EG-Verwaltung durch Ausgründung etwa Priebe Entscheidungsbefugnisse (Fn. 13), 15ff.; Kugelmann (Fn. 45), Art. 218 Rn. 15 ff.; M. H. Koch Die Externalisierungspolitik der Kommission, 2004, 105 ff.; allgemein zum neu entstehenden Agentursystem E. Chiti Le Agenzie Europee, 2002, 315 ff.; Fischer-Appelt Agenturen (Fn. 91), 218ff.; Sydow Externalisierung (Fn. 95), 1 ff. mwN. 
Exekutiven dar, durch den die nationalen Verwaltungen bereits in den Prozess der Verabschiedung der später von ihnen anzuwendenden Gemeinschaftsrechtsakte eingebunden ${ }^{126}$ und an der Vollzugsüberwachung beteiligt werden. ${ }^{127}$ In den letzten Jahren ist außerdem die institutionalisierte Kooperation in der Eigenverwaltung der EU erheblich intensiviert worden. ${ }^{128}$ Inzwischen besteht eine Vielzahl verselbständigter dezentraler Verwaltungseinrichtungen, ${ }^{129}$ die als Europäische Ämter und Agenturen integrale Bestandteile der institutionell ausdifferenzierten EG-Eigenverwaltung bilden ${ }^{130}$ und innerhalb derer - insbesondere in den Regulierungsagenturen ${ }^{131}$ - Vertreter der Mitgliedstaaten über die

126 So $T$. Gro $\beta \S 13$ Verwaltungsorganisation, in: Hoffmann-Riem/Schmidt-Aßmann/ Voßkuhle (Fn. 12), Rn. 110 mwN.

${ }^{127}$ In diesem Sinne $R$. Priebe Europäische Rechtsetzung und mitgliedstaatliche Beteiligung: die Sicht der EU-Kommission, in: H. Siedentopf (Hrsg.) Der europäische Verwaltungsraum, 2004, 91 (93); die seit 1962 geübte Praxis der Komitologieausschüsse ist heute in Art. 202 Spiegelstrich 3 S. 2 EGV primärrechtlich anerkannt, vgl. ausführlich zum rechtlichen Rahmen der Komitologie G. Roller Komitologie und Demokratieprinzip, KritV 2003, 249 (267ff. mwN); nach Art. 202 EGV darf der Rat, wenn er der Kommission die Durchführung der von ihm erlassenen Vorschriften überträgt, die Modalitäten für die Ausübung dieser Befugnisse festlegen. Diese Modalitäten sind festgelegt durch den Beschluss 1999/468/EG vom 28. Juni 1999, AB1. EG 1999 Nr. L 184, 23, dazu: M. Gellermann in: Streinz (Fn. 9), vor Art. 250 Rn. 20 ff.; M. Hauschild Das neue Komitologieverfahren, ZG 1999, $248 \mathrm{ff}$. mwN; die Komitologieausschüsse besitzen zwar keine eigenen Entscheidungs- oder Zustimmungskompetenzen, je nach ihrer Beschlussfassung ist aber bei Meinungsverschiedenheiten zwischen Kommission und Ausschuss der Rat wieder am Erlass der an sich der Kommission übertragenen Durchführungsbestimmungen zu beteiligen, vgl. ebd., 249 (250f.).

128 Eine systematisierende Darstellung hierzu gibt etwa C. Franchini RTDP 2002, 603 ff.; Grundlage dieser Ausdifferenzierung ist eine gezielte Externalisierungspolitik der Europäischen Kommission, vgl. hierzu die Mitteilung „Externalisierung der Verwaltung der Gemeinschaftsprogramme“, KOM (2000) 788, 4 ff. sowie Sydow Externalisierung (Fn. 95), 1 f. mwN.

129 Die nicht im Primärrecht vorgesehenen Institutionen sind zunächst als ,vertragsfremde Einrichtungen“ bezeichnet worden, so bereits Priebe Entscheidungsbefugnisse (Fn. 13); vgl. auch Berger Einrichtungen (Fn. 14) mit Überblick über die Entwicklungsphasen der Gründung rechtsfähiger Einrichtungen ebd., $31 \mathrm{ff}$. mwN.

130 Sydow Externalisierung (Fn. 95), 1 (2) mwN; ausführlich zu Begriff, Merkmalen und Typologie der europäischen Agenturen Fischer-Appelt Agenturen (Fn. 91), $38 \mathrm{ff}$. mwN.

131 Ausführlich zu den Regulierungsagenturen Koch Externalisierungspolitik (Fn. 125), 56 ff.; grundsätzlich zur Unterscheidung zwischen Exekutiv- und Regulierungsagenturen Sydow Verwaltungskooperation (Fn. 6), $65 \mathrm{ff}$. mwN; ders. Externalisierung und institutionelle Ausdifferenzierung, VerwArch 97 (2006), 4 ff. mwN; T. Groß (Fn. 17), 54 (56ff. mwN); Exekutivagenturen sind von der Kommission auf der Grundlage einer Ratsverordnung errichtete juristische Personen des Gemeinschaftsrechts, die für Rech- 
Leitungsgremien maßgeblichen Einfluss auf die Verwaltungstätigkeit der Gemeinschaftseinrichtungen nehmen können. ${ }^{132}$

\section{Wertung}

Damit durchziehen Kooperation und Verflechtung als Modi der Verantwortungswahrnehmung auf der Grundlage sekundärrechtlicher Vorgaben des Gemeinschaftsgesetzgebers sämtliche Formen des Verwaltens im Verhältnis von EU und Mitgliedstaaten. Die europäische Mehrebenenverwaltung ist nicht auf Trennung der Verwaltungskompetenzen und Betonung der organisatorischen Eigenständigkeit von EU und Mitgliedstaaten ausgerichtet, sondern betont durch die flexiblen sekundärrechtlichen Kompetenzzuweisungen und -verschränkungen die Variabilität der Aufgabenwahrnehmung im Mehrebenensystem und zugleich die funktionale Verbundenheit und Angewiesenheit der verschiedenen Ebenen aufeinander.

Im Interesse einer effektiven und effizienten Gemeinschaftsverwaltung findet nach sachgebietsspezifischen Notwendigkeiten in unterschiedlicher Intensität eine Teilung oder präziser eine funktionsdifferenzierende gemeinsame Wahrnehmung der Verwaltungskompetenzen statt, die dem offenen Verbundcharakter der Mehrebenenverwaltung entspricht und von beiden Ebenen je unterschiedliche Beiträge zur Erfüllung der gemeinsamen Verwaltungsverantwortung einfordert.

Klare, einheitlichen Kriterien und Baumustern folgende und durchgängig verwirklichte Verantwortungsstrukturen lassen sich in diesem System allerdings nur schwerlich erkennen.

nung und unter Aufsicht der Kommission ein gemeinschaftliches Programm oder Vorhaben ganz oder teilweise durchführen, so die Definition in Art. 55 Abs. 1 der VO 1605/2002 des Rates vom 25. Juni 2002 über die Haushaltsordnung für den Gesamthaushaltsplan der Europäischen Gemeinschaften; die Exekutivagenturen besitzen zwar eine gewisse operative Verwaltungsautonomie, sind aber durch die Vorgaben der Ratsverordnung zur Festlegung des Statuts der Exekutivagenturen sehr eng an die Kommission angebunden, vgl. VO (EG) Nr. 58/2003 des Rates v. 19. 12. 2002 zur Feststellung des Status der Exekutivagenturen, die mit bestimmten Aufgaben bei der Verwaltung von Gemeinschaftsprogrammen beauftragt werden, AB1 EG 2003, Nr. L 11, 1.

${ }^{132}$ Hierzu ausführlich Fischer-Appelt Agenturen (Fn. 91), $220 \mathrm{ff} . \mathrm{mwN}$; Trute Legitimation (Fn. 37), Rn. 109; zu den einzelnen Kooperationsformen innerhalb der Regulierungsagenturen Gro $\beta$ (Fn. 17), 54 (58ff. mwN); Sydow Externalisierung (Fn. 95), 1 (5) mwN; Trute Legitimation (Fn. 37), Rn. 109. 


\section{Demokratische und rechtsstaatliche Verantwortung}

Entspricht nun diese europäische Mehrebenenverwaltung und die Verantwortungsverteilung in ihr den Vorgaben des europäischen Verfassungsverbundes für europäisches Verwalten?133

Die wesentlichen Konstitutionsprinzipien für die Ausgestaltung von Verwaltung im europäischen Mehrebenensystem sind - über die Einhaltung der Kompetenzordnung hinaus - insbesondere das Demokratieprinzip und der Grundsatz der Rechtsstaatlichkeit. ${ }^{134}$ Die europäische Verwaltung und auch die Verantwortungsverteilung in ihr müssen sich an den grundlegenden Anforderungen von Demokratie und Rechtsstaatlichkeit an jede Hoheitsausübung messen lassen. ${ }^{135}$ Ist also die europäische Mehrebenenverwaltung in ihrer gegenwärtigen Konstruktionsform eine Art der Organisation und verfahrensmäßigen Ausgestaltung der Wahrnehmung von Verwaltungsaufgaben, die den Anforderun-

${ }^{133}$ Die Vorgaben der Verfassung für die Verwaltung in ihren unterschiedlichen Dimensionen betont etwa Schmidt-Aßmann, Ordnungsidee (Fn. 6), 393: „Dem realen Verwaltungsverbund entspricht ein normativer Verfassungsverbund. Die zur Verwaltung des Gemeinschaftsraumes notwendigen administrativen Verflechtungen erhalten durch diese Verfassung ihre normative Absicherung, Ausrichtung und Begrenzung“.

${ }^{134} \mathrm{Zu}$ diesen Grundanforderungen Winter Kompetenzverteilung (Fn. 5), 255 (259ff., $267 \mathrm{ff}$., jeweils mwN), dort auch kritisch dazu, dass die Kommission in ihrem Weißbuch von einer eher politischen denn rechtlichen Verpflichtung zur durch das demokratische Prinzip vorgegebenen Steigerung von Transparenz und Partizipation ausgehe, vgl. ders. ebd., 267 mwN sowie Weißbuch „Europäisches Regieren“, KOM (2001) 428 endg. vom 25. 07. 2001, 13: Offenheit, Partizipation, Verantwortlichkeit und Effektivität als zentrale Anforderungen als Grundsätze guten Regierens in Europa; zur zentralen Bedeutung von Demokratie und Rechtsstaatlichkeit neben Effizienz und Subsidiarität für die Ausgestaltung des europäischen Verwaltungsverbundes auch Schmidt-Aßmann, Ordnungsidee (Fn. 6), 393f. mwN.

135 Diese demokratischen und rechtsstaatlichen Anforderungen sind zentrale Bestandteile nicht nur der Verfassungsordnung der EU, sondern ebenso aller in ihr verbundener Mitgliedstaaten; Art. 6 Abs. 1 EUV stellt ausdrücklich klar, dass nicht nur die EU auf den Grundsätzen der Demokratie und der Rechtsstaatlichkeit beruht, sondern dass diese Grundsätze ebenso allen Mitgliedstaaten gemeinsam sind; zur Demokratie als Verfassungsgrundsatz der EU und zum unterschiedlichen Demokratieverständnis in den Mitgliedstaaten J. Gerkrath Die Bedingungen der Demokratie in der Europäischen Union / Ein französischer Standpunkt, EuGRZ 2006, $371 \mathrm{ff}$. mwN, dort mit dem anschaulichen (zitierten) Hinweis auf die Anreicherung von Demokratie mit unterschiedlichen Werten, etwa „Verfechtung der Transparenz für die Skandinavier, soziale Sicherung für die rheinischen Länder, soziale und ökonomische Kohäsion sowie öffentliche Dienste für die südlichen Länder, ... Grundrechte und Nicht-Diskriminierung für alle“, ders. ebd., 372 sowie Fn. 9; zur Variabilität des Demokratiebegriffes und zu den demokratischen Mindestanforderungen des Art. 6 Abs. 1 EUV auch M. Pechstein in: Streinz (Fn. 9), Art. 6 EUV Rn. 5. 
gen genügt, die aus dem Demokratieprinzip und dem Rechtsstaatsprinzip folgen? ${ }^{136}$

\section{Anforderungen des Demokratieprinzips}

Aus dem Demokratieprinzip folgt insbesondere das Erfordernis hinreichender demokratischer Legitimation. ${ }^{137}$ Mittlerweile wird zunehmend hervorgehoben, dass nicht nur die europäische Rechtsetzung, sondern ebenso die europäische Mehrebenenverwaltung und das europäische Verwaltungshandeln einer ausreichenden demokratischen Legitimation im Sinne einer hinreichenden Rückbindung der Herrschaftsausübung an den Willen der europäischen Herrschaftsunterworfenen bedürfen. 138

Problematisch ist, dass die traditionellen monistischen repräsentativdemokratischen Erwartungen an Verwaltungslegitimation von der europäischen Mehrebenenverwaltung nicht erfüllt werden. ${ }^{139}$ Diese regelmäßig

${ }^{136}$ Heute ist allgemein anerkannt, dass eine der Grundanforderungen des Demokratieprinzips eine Verfahrensgestaltung für die Ausübung von Herrschaftsgewalt ist, die den Anforderungen an demokratische Legitimation möglichst gut Rechnung trägt, vgl. nur S. Kadelbach Bedingungen einer demokratischen Europäischen Union / Ein deutscher Standpunkt, EuGRZ 2006, 384, der sich dort auf die Titandioxid-Entscheidung des EuGH stützt, in der der Gerichtshof entsprechende Erwägungen allerdings spezifisch für die gemeinschaftliche Rechtsetzung und die Auswahl der für einen Rechtsakt einschlägigen Rechtsgrundlage auch nach der Art der Beteiligung des Europäischen Parlaments angestellt hat, vgl. EuGH Slg. 1991, I-2867 Rn. 20; das Demokratie- und das Rechtsstaatsprinzip gebieten zusammenfassend eine prinzipiengerechte Optimierung der Verantwortungsverteilung im europäischen Mehrebenensystem.

${ }^{137}$ Die demokratische Legitimation von Staats- und Hoheitsgewalt ist lange Zeit unspezifisch mit Blick auf die Staatsgewalt insgesamt oder spezifisch im Hinblick auf die Rechtsetzung diskutiert worden. Erst in jüngerer Zeit, mit zunehmender Anerkennung von Eigenständigkeit und eigenem politischem Gestaltungsspielraum der Verwaltung, findet das Gebot der eigenständigen demokratischen Legitimation der Verwaltung gröBere Aufmerksamkeit; ausführlich zu den Ursachen, die in der zunächst allzu sehr betonten Ausrichtung der Diskussion über die Verwaltung auf parlamentarische Steuerung durch das Gesetz und rechtsstaatliche Einbindung liegen, Trute Legitimation (Fn. 37), Rn. 3 mwN.

138 So deutlich etwa Schulze-Fielitz Verfassungsgefüge (Fn. 13), $127 \mathrm{ff}$. mwN; ausführlich zur Legitimationsbedürftigkeit der europäischen supranationalen Hoheitsgewalt H.-G. Dederer Korporative Staatsgewalt, 2004, S. 586 ff. mwN, sowie G. Hermes, Legitimationsprobleme unabhängiger Behörden, in: H. Bauer/P. M. Huber/K.-P. Sommermann (Hrsg.) Demokratie in Europa, 2005, 457 (467 ff.); zur angeblichen gegenwärtigen Legitimationskrise der EG-Organe vgl. etwa $K$. $O$. Nass Legitimität und Identität der Europäischen Union, DVB1. 2006, 1197 (1198f. mwN).

${ }^{139}$ Nach Schliesky Souveränität (Fn. 2), 389, „spricht vieles dafür, der supranationalen Herrschaftsgewalt bei Zugrundelegung des herrschenden deutschen Legitimations- 
an die nationale Verwaltung gestellten Legitimationsanforderungen ${ }^{140}$ erfordern grundsätzlich eine ununterbrochene Legitimationskette zwischen Volk und Verwaltungshandlung, die über Wahlen, Ernennungen und Verwaltungshierarchie die Verantwortlichkeit der Verwaltung für ihre Handlungen vor der Gesamtheit aller Bürger bewirkt. ${ }^{141}$ Danach erfolgt demokratische Legitimation in drei Legitimationsmodi:142 institutionell-funktionell durch eine verselbständigte Exekutive, organisatorisch-personell durch Amtswalter, die ihre Befugnisse durch einen ununterbrochenen Legitimationszusammenhang auf den Volkswillen zurückführen können, und sachlich-inhaltlich durch Bindung an die Gesetze. ${ }^{143}$ Dabei ist nicht eine bestimmte Form oder Intensität der Verwirklichung der einzelnen Legitimationsformen erforderlich, sondern ein hinreichendes Legitimationsniveau, das durch bausteinartiges $\mathrm{Zu}$ sammenwirken der verschiedenen Legitimationsformen bewirkt werden kann. ${ }^{144}$

konzepts die ausreichende demokratische Legitimation bzw. Legitimität abzusprechen“; nicht näher einzugehen ist hier auf die mittlerweile überwiegend akzeptierten Überlegungen zur grundsätzlich zweigleisigen Legitimationsstruktur der EU, deren Legitimationsbasis einerseits in den nationalen Parlamenten der Mitgliedstaaten und von diesen ausgehenden Legitimationssträngen, andererseits im Legitimationsbeitrag des Europäischen Parlamentes gesehen wird, hierzu nur etwa W. Hertel Supranationalität als Verfassungsprinzip, 1999, $165 \mathrm{ff}$. mwN, sowie C. Callies Optionen zur Demokratisierung der EU, in: Bauer/Huber/Sommermann (Fn. 138), 281 (282 ff. mwN), da auch dieser Legitimationsansatz die spezifischen Probleme der Legitimation der Mehrebenenverwaltung mit ihren multiplen zusätzlichen Aufgaben- und Kompetenzverflechtungen nicht im Sinne repräsentativdemokratischer Legitimationserwartungen bewältigen kann.

140 Schulze-Fielitz Verfassungsgefüge (Fn. 13), 127, betont, dass diese Konstruktion von Legitimation „nicht nur nach deutschem Verständnis“ jedenfalls die auf nationaler Ebene geforderte Form der Rückbindung von Herrschaft an den Volkswillen darstellt.

141 So dezidiert G. Lübbe-Wolff Europäisches und nationales Verfassungsrecht, VVDStRL 60 (2001), 246 (280 mwN), die vom „im Topos der „nunterbrochenen Legitimationskette“ verdichteten repräsentativdemokratischen Legitimationsmodell“" spricht.

${ }^{142} \mathrm{Zu}$ den Modi, also den zulässigen Mitteln der Bewirkung demokratischer Verwaltungslegitimation im von ihm so bezeichneten „klassischen Legitimationsmodell“ näher Trute Legitimation (Fn. 37), Rn. $7 \mathrm{ff}$.

${ }^{143}$ Vgl. ausführlicher zu diesem „klassischen“ Modell Trute Legitimation (Fn. 37), Rn. 7 ff.; H. Dreier, in: ders. (Fn. 70), Art. 20 (Demokratie) Rn. 104ff. mwN; Hermes Legitimationsprobleme (Fn. 138), 467 ff.; als ,input-orientierte“ Legitimation (zuletzt) bei F.W. Scharpf $\S 20$ Legitimationskonzepte jenseits des Nationalstaats, in: G. F. Schuppert/I. Pernice/U. Haltern (Hrsg.) Europawissenschaft, 2005, 705 (709ff., 717 ff.); ferner ausf. Schliesky Souveränität (Fn. 2), $230 \mathrm{ff}$.

${ }^{144}$ Ausführlich Trute Legitimation (Fn. 37), Rn. 14; es kann also je nach der Art des Zusammenwirkens der verschiedenen Formen von Legitimation ein „hinreichendes Le- 
Dieses Modell der Legitimation der Verwaltung stößt bereits beim rein nationalen Verwaltungshandeln einer heute differenzierten und pluralisierten Verwaltung auf zahlreiche Probleme und Durchbrechungen. ${ }^{145}$ Für die Legitimation des Verwaltungshandelns im europäischen Verwaltungsverbund muss es versagen: Es kann weder die Vielfalt der an der europäischen Verwaltung beteiligten administrativen Akteure noch die angesichts dieser Vielfalt relevanten unterschiedlichen, teils verflochtenen und teils gegenläufigen Legitimationsstränge noch die qualitativ heterogene Zusammensetzung instrumentalisierter Legitimationsbausteine in Europa angemessen erfassen. ${ }^{146}$

Unabhängig vom zugrunde zu legenden Legitimationskonzept ${ }^{147}$ verlangt das europäische Demokratieprinzip jedenfalls ein angemessenes $\mathrm{Maß}$ an demokratischer Legitimation. Diese demokratische Legitimation muss aber für die europäische Verwaltung auch in anderer Weise, durch Anerkennung anderer zusätzlicher Legitimationsbausteine geleistet werden können, als dies nach allein repräsentativ-demokratischen

gitimationsniveau“ auf unterschiedliche Weise gewährleistet werden, vgl. BVerfGE 93, 37 (66); 83, 60 (72), zu dessen Erreichung sich die verschiedenen Legitimationsmodi ergänzen und untereinander ausgleichsfähig, aber nicht gänzlich ersetzbar sind, hierzu nur Schmidt-Aßmann, Ordnungsidee (Fn. 6), 99 f. mwN.

145 Ebenso Schulze-Fielitz Verfassungsgefüge (Fn. 13), 128f. mwN; zu Kritik und Alternativen näher Trute Legitimation (Fn. 37), Rn. 115 ff. mwN; vgl. auch H. Bauer Demokratie in Europa - Einführende Problemskizze, in: Bauer/Huber/Sommermann (Fn. 138), 1 (3 mwN), der dort etwa auf ministerialfreie Räume, mittelbare Staatsverwaltung wie funktionale und kommunale Selbstverwaltung oder verselbständigte Verwaltungseinrichtungen hinweist; B.-O. Bryde Die bundesrepublikanische Volksdemokratie als Irrweg der Demokratietheorie, Staatswissenschaften und Staatspraxis 5 (1994), 305 (324) spricht von „Legitimationskettenfetischismus“; kritisch auch Hermes Legitimationsprobleme (Fn. 138), 469 ff.; umfassend Schliesky, Souveränität (Fn. 2), $389 \mathrm{ff}$.

146 In diesem Sinne auch W. Hoffmann-Riem Strukturen des Europäischen Verwaltungsrechts - Perspektiven der Systembildung, in: E. Schmidt-Aßmann/W. HoffmannRiem (Hrsg.) Strukturen des Europäischen Verwaltungsrechts, 1999, 317 (376f.); Nehl Europäisches Verwaltungsverfahren (Fn. 7), $120 \mathrm{ff}$. mwN; ausführlich zum Ganzen Trute Legitimation (Fn. 37), Rn. $102 \mathrm{ff} . \mathrm{mwN}$; näher auch Winter Kompetenzverteilung (Fn. 5), 255 (268f. mwN).

147 Schliesky Souveränität (Fn. 2), 389 f. mwN, betont zutreffend, dass es bei der Kritik am angeblichen Demokratiedefizit der EU häufig eher um die Ergebnisse der Beurteilung supranationaler Hoheitsgewalt am Maßstab der herrschenden deutschen Legitimationsvorstellungen geht; auf für die Legitimation europäischer Hoheitsgewalt vorstellbare Alternativkonzepte wie etwa die Gleichheit der Aktivbürger, deren tendenzielle Selbstregulierung, ihre qualifizierte Mitwirkung oder einen sog. Elitenwettbewerb mit bürgerschaftlicher Sanktionsgewalt, weist $M$. Ruffert Demokratie und Governance in Europa, in: Bauer/Huber/Sommermann (Fn. 138), 319 (341) in Anlehnung an A. v. Bogdandy Europäische Prinzipienlehre, in: von Bogdandy (Fn. 2), 149 (181), hin. 
Legitimationstheorien der Fall ist. ${ }^{148}$ Für die demokratische Legitimation der Verantwortungsverteilung und der Handlungen des europäischen Verwaltungsverbundes kann zunächst als ein zusätzlicher Legitimationsbaustein die grundsätzliche strukturelle demokratische Legitimation der europäischen Mehrebenenverwaltung herangezogen werden, die sich aus der allen an der Verwaltung des Gemeinschaftsraums beteiligten Verwaltungen gemeinsamen demokratischen und rechtsstaatlichen Verwaltungsstruktur ergibt. ${ }^{149}$ Dieser Legitimationsansatz kann ergänzt werden durch partizipative Legitimationselemente, durch eine Ausgestaltung der Verwaltungsverfahren im europäischen Verwaltungsverbund, die eine möglichst weitreichende Partizipation nicht nur der unmittelbar Betroffenen, sondern auch Drittbetroffener und interessierter Dritter ermöglicht und die bislang dominierenden kollektivistischen Legitimationsansätze um individualistisch-freiheitssichernde Legitimationselemente ergänzt. ${ }^{150}$

Hinzukommen muss im verflochtenen europäischen Verwaltungsverbund als zentrale Grundanforderung demokratischer Verantwortlichkeit Legitimation durch Transparenz und Verantwortungsklarheit, die erst die eindeutige Zuordnung einzelner Verfahrensschritte und Entscheidungsteile zu einzelnen Ebenen und Verwaltungseinrichtungen der Mehrebenenverwaltung und dadurch die Zuordnung von Verwaltungsergebnissen zu Verwaltungsträgern ermöglichen. ${ }^{151}$

148 Auf die unterschiedlichen in der europäischen Legitimationsdebatte diskutierten partizipativen, verfahrens- oder output-orientierten sowie deliberativen Legitimationsansätze weist zu Recht Sydow Verwaltungskooperation (Fn. 6), 239ff. mwN, hin; für einen Überblick über die europäische Legitimitätsdiskussion vgl. auch A. Peters Elemente einer Theorie der Verfassung Europas, 2001, $499 \mathrm{ff}$. mwN; deutlich zur erforderlichen abweichenden Legitimationskonzeption für die EU Scharpf Legitimationskonzepte (Fn. 143), 705 (723 ff. mwN).

149 Zur gemeinsamen Verpflichtung und Ausrichtung der EU und aller Mitgliedstaaten auf Demokratie und Rechtsstaatlichkeit vgl. Art. 6 Abs. 1 EUV sowie hierzu M. Hilf/F. Schorkopf in: Grabitz/Hilf (Fn. 50), Art. 6 EUV Rn. 6 ff. mwN, dort auch zur Legitimationsfunktion dieser Homogenitätsklausel; Trute Legitimation (Fn. 37), Rn. 114 spricht insoweit von einer „Basislegitimation des Europäischen Verwaltungsraums“.

150 Hinweise auf die vorstellbare „Pluralität legitimationsstiftender Faktoren“ gibt Schulze-Fielitz Verfassungsgefüge (Fn. 13), $130 \mathrm{f}$. mwN; instruktiv auch M. Nettesheim Demokratisierung der Europäischen Union und Europäisierung der Demokratietheorie - Wechselwirkungen bei der Herausbildung eines europäischen Demokratieprinzips, in: Bauer/Huber/Sommermann (Fn. 138), 143 (180ff. mwN).

${ }^{151}$ Dies betont als zentrale Grundanforderung an Legitimation insbesondere in den Verbundstrukturen der europäischen Mehrebenenverwaltung deutlich Schmidt-Aßmann Verwaltungskooperation (Fn. 10), 296f,; zur Verantwortungsklarheit auch Sydow Verwaltungskooperation (Fn. 6), $241 \mathrm{f} . \mathrm{mwN}$. 
Schließlich kann auch die Effizienz der Verwaltung als output-orientierter Legitimationsansatz Verwaltungshandeln und Verantwortungsverteilung in der europäischen Mehrebenenverwaltung ergänzend legitimieren, weil Teil des demokratisch geforderten Verwaltungshandelns auch die bestmögliche und effektive Erledigung der übertragenen Gemeinwohlaufgaben ist. ${ }^{152}$

Angesichts dieser Vielfalt sich ergänzender Legitimationsbausteine folgt aus dem Demokratieprinzip ein an den europäischen Kooperationsgesetzgeber gerichtetes Optimierungsgebot dahin, Verantwortung im europäischen Mehrebenensystem so zu verteilen, dass sie im strukturell grundsätzlich demokratisch legitimierten europäischen Verwaltungsgefüge klar und eindeutig zugeordnet ist, dass Transparenz über Zuständigkeiten und Verfahren besteht, dass die Partizipation Betroffener und Interessierter am Verfahren ermöglicht wird und dass im Rahmen dieser Vorgaben die Verwaltungsaufgaben effizient wahrgenommen werden können.

\section{Rechtsstaatliche Anforderungen}

Darüberhinaus muss die Verantwortungszuweisung in der europäischen Verwaltung rechtsstaatlichen Anforderungen genügen. ${ }^{153}$ Zentrale Anforderungen der Rechtsstaatlichkeit sind insoweit zumindest Verantwortungsklarheit, effektiver Rechtsschutz und eindeutige Haftung. ${ }^{154}$

Durch die Zusammenarbeit von Verwaltungsinstanzen der EG ${ }^{155}$ und der Mitgliedstaaten bei der Erledigung einzelner Verwaltungsvorgänge -

\footnotetext{
152 In diesem Sinne deutlich Schliesky Souveränität (Fn. 2), 659 ff. mwN, der dort ausführlich die Tauglichkeit output-orientierter Elemente zur Legitimation behandelt.

${ }^{153}$ Zur Rechtsstaatlichkeit der EU und ihren unionsspezifischen Dimensionen näher D. H. Scheuing $\S 6$ Rechtsstaatlichkeit, in: Schulze/Zuleeg (Fn. 5), Rn. 1 ff. mwN, dort zu den Anforderungen des Grundsatzes effektiven Rechtsschutzes näher in Rn. 49 ff.; ausführlich zur Bedeutung der Rechtsstaatlichkeit im Rahmen der Homogenitätsklausel des Art. 6 Abs. 1 EUV auch Hilf/Schorkopf (Fn. 149), Art. 6 EUV Rn. 27 ff. mwN.; zum gemeinschaftlichen Rechtsprinzip und seiner Bedeutung für die Erforderlichkeit effektiver Rechtsschutzmöglichkeiten auch ausführlich E. Pache Tatbestandliche Abwägung und Beurteilungsspielraum, 2001, $309 \mathrm{ff}$. mwN.

154 Ausführlicher hierzu J. Hofmann Rechtsschutz und Haftung im Europäischen Verwaltungsverbund, in: Schmidt-Aßmann/Schöndorf-Haubold (Fn. 17), 353 (359ff., $376 \mathrm{ff}$., jeweils mwN); ders. Rechtsschutz und Haftung im Europäischen Verwaltungsverbund, 2004, $163 \mathrm{ff}$. mwN.

155 Auf spezifische Rechtsschutzprobleme beim Verwaltungshandeln europäischer Agenturen weist T. Gro $\beta$ Die Kooperation zwischen europäischen Agenturen und nationalen Behörden, EuR 2005, 54 (65f. mwN), hin.
} 
seien es Produktzulassungen, Schutzgebietsausweisungen ${ }^{156}$ oder Beihilfeentscheidungen - kann für die Adressaten der Verwaltungsmaßnahmen der Verantwortungsanteil, den die verschiedenen Verwaltungseinheiten an der letztlich erlassenen Maßnahme zu tragen haben, nicht eindeutig erkennbar sein. Die Überprüfung präjudiziell relevanter Handlungen verfahrensbeteiligter Behörden anderer Mitgliedstaaten oder anderer Ebenen des Mehrebenensystems kann Fragen der Rechtswegklarheit und der Zumutbarkeit der Rechtsverfolgung aufwerfen, und die begrenzte Jurisdiktionsgewalt nationaler Gerichte gegenüber Handlungen der Gemeinschaftsinstitutionen ist insoweit ebenso problematisch $^{157}$ wie der nur begrenzte Zugang Einzelner unmittelbar zur Gemeinschaftsgerichtsbarkeit. ${ }^{158}$

Rechtsstaatlich bedenklich sind Unklarheiten über den einzuschlagenden Rechtsweg ebenso wie darüber, wer für eventuelle Fehler einer Entscheidung die Verantwortung im Sinne einer Haftung zu übernehmen hat. Diese bei Kooperation zwischen unabhängigen Verwaltungseinheiten stets auftretenden Unklarheiten und Abstimmungsprobleme gewinnen in der europäischen Mehrebenenverwaltung besonderes Gewicht dadurch, dass sowohl das Rechtsschutzsystem als auch das Haftungssystem der Verträge ungeachtet der realen Vielfalt von Verwaltungskooperation und -verschränkung grundsätzlich am Trennungsprinzip bezüglich Rechtsschutz und Haftung festhalten. ${ }^{159}$

Hier ist rechtsstaatlich zumindest geboten, mit den materiellen Kooperationsvorschriften sachgebiets- und verfahrensspezifisch eindeutige Rechtsschutz- und Haftungsregelungen aufzustellen ${ }^{160}$ und die Betroffe-

156 Die Probleme im Verhältnis zwischen nationalem und europäischem Rechtsschutz bei der kooperativen Konzeptentwicklung nach der FFH-Richtlinie behandelt exemplarisch Weiß Schnittstellenprobleme (Fn. 2) 517 (537 ff. mwN).

$157 \mathrm{Zu}$ den nur begrenzten nationalen Rechtschutzmöglichkeiten Weiß Schnittstellenprobleme (Fn. 2), 517 (536f. mwN), dort auch zur aus dem Trennungsprinzip folgenden möglichen Aufspaltung zusammenhängender Sachverhalte und Rechtsschutzfragen; gefordert wird deshalb zutreffend ein angemessen auf die Verbundprobleme reagierender, „kohärenter“ Rechtsschutz, der über die Möglichkeit der Kooperation nationaler Gerichte mit der unmittelbar europäischen Gerichtsbarkeit hinausgehen muss, vgl. ausführlicher Schmidt-Aßmann Verfassungsprinzipien (Fn. 12), Rn. $80 \mathrm{ff}$. mwN.

${ }^{158}$ Hierzu ausführlich E. Pache Rechtsschutzdefizite im europäischen Grundrechtsschutz, in: T. Bruha/C. Nowak/H. Petzold (Hrsg.) Grundrechtsschutz für Unternehmen im europäischen Binnenmarkt, Baden-Baden 2004, 193 ff. mwN.

159 Kritisch hierzu auch Schmidt-Aßmann Verfassungsprinzipien (Fn. 12), Rn. 83; Hofmann Haftung im Europäischen Verwaltungsverbund (Fn. 154), 163 ff. mwN.

160 Wie dies etwa für das Zollinformationssystem in Art. 40 der VO (EG) Nr. 515/97 vom 13. 3. 1997, ABl. EG Nr. L 82 S. 1, bereits vorgesehen ist, vgl. hierzu Schmidt-Aßmann Strukturen (Fn. 17), 408 mwN. 
nen des kooperativen Verwaltungshandelns hierüber ausführlich zu belehren und $\mathrm{zu}$ informieren. ${ }^{161}$

\section{Perspektiven}

Welches Gesamtbild von Verantwortung und Effizienz in der Mehrebenenverwaltung ergibt sich hieraus?

Die Aufgaben und Befugnisse der Verwaltung werden im europäischen Mehrebenensystem durch das Primärrecht weder der EU noch ihren Mitgliedstaaten starr und eindeutig zugewiesen. Vielmehr übertragen die funktional ausgerichteten Gemeinschaftskompetenzen dem Gemeinschaftsgesetzgeber die Aufgabe, je nach sachgebietsspezifischer Notwendigkeit in den Grenzen des Subsidiaritätsprinzips und des Verhältnismäßigkeitsgrundsatzes über die Zuweisung der Verwaltungsverantwortung zu entscheiden. Zulässig und unter Effizienzgesichtspunkten sinnvoll ist dabei in weiten Bereichen auch die gemeinsame arbeitsteilige Wahrnehmung von Verwaltungsverantwortung durch Zusammenarbeit von Verwaltungseinrichtungen der verschiedenen Ebenen oder innerhalb einer Ebene.

Bei der durch diese flexible Verantwortungsstruktur ermöglichten sekundärrechtlichen Verantwortungsverteilung muss der Gemeinschaftsgesetzgeber die demokratischen und rechtsstaatlichen Anforderungen der europäischen Verfassung an den europäischen Verwaltungsverbund berücksichtigen. Aus diesen folgen Optimierungsgebote für die demokratische und rechtsstaatliche Ausgestaltung der Institutionen und Verfahren der Verantwortungswahrnehmung: Bei der sekundärrechtlichen Ausgestaltung der konkreten Verantwortungsverteilung in der europäischen Mehrebenenverwaltung sind deshalb so weit wie möglich Transparenz der Verfahren und Verantwortlichkeiten, Partizipationsmöglichkeiten für die Betroffenen und die Öffentlichkeit, Verwirklichung der rechtsstaatlichen Gebote der Verantwortungsklarheit und des effektiven Rechtsschutzes sowie Effizienz der Aufgabenwahrnehmung anzustreben. Dieser komplexe Optimierungsauftrag an den europäischen Gesetzgeber stellt die erforderliche demokratisch-rechtsstaatliche Einhegung der großen Flexibilität der primärrechtlichen Verantwortungsverteilung zwischen den oberen Ebenen der europäischen Mehrebenenverwaltung sicher.

\footnotetext{
${ }^{161}$ In Betracht kommt etwa die Konstruktion einer konzentrierenden Stellvertreterhaftung, in diesem Sinne Hofmann Rechtsschutz (Fn. 154), $353 \mathrm{ff}$.
} 
Erwägenswert erscheint, ob die Verwirklichung der demokratischen und rechtsstaatlichen Anforderungen an die Verantwortungsverteilung durch den Versuch der sekundärrechtlichen Kodifikation einer Typik von Kooperationsformen gefördert werden könnte, die der europäische Gesetzgeber bereichsspezifisch je nach den festgestellten Kooperationsbedürfnissen für die sachlich angemessene Verantwortungszuweisung innerhalb der europäischen Mehrebenenverwaltung einsetzen könnte. Bereits der Versuch einer solchen Kodifikation strukturierter Typen von Verwaltungsverantwortung und Verwaltungskooperation, eines demokratisch und rechtsstaatlich ausgestalteten allgemeinen Teils des europäischen Verwaltungskooperationsrechts mit Regelungen zu Verantwortlichkeiten, Beteiligungsrechten, Informations-, Belehrungs- und Begründungspflichten sowie zu Rechtsschutz und Haftung bei den unterschiedlichen Modi der Verantwortungswahrnehmung im europäischen Verwaltungsverbund könnte auf europäischer und auf nationaler Ebene jedenfalls das Bewusstsein um die demokratischen und rechtsstaatlichen Probleme und Anforderungen der Verwaltungskooperation in der Mehrebenenverwaltung deutlich schärfen.

Unabhängig hiervon ist bereits gegenwärtig trotz aller tatsächlichen Notwendigkeit von Verwaltungskooperation und trotz der großen rechtlichen Flexibilität der europäischen Kompetenzordnung nicht Effizienz allein der Maßstab für Verantwortung im europäischen Mehrebenensystem. Nur eingebunden in die grundlegenden demokratischen und rechtsstaatlichen Anforderungen des europäischen Verfassungsverbundes können auch Effizienzgesichtspunkte Bedeutung für die Verantwortungsverteilung in der europäischen Mehrebenenverwaltung erlangen. 
Leitsätze des 1. Berichterstatters über:

\section{Verantwortung und Effizienz in der Mehrebenenverwaltung}

\section{Begriffsbestimmung}

1. Die Verwaltung der Europäischen Union erfolgt in einem föderativ strukturierten Mehrebenensystem, das sich als funktional bei der Konkretisierung und Verwirklichung des europäischen Gemeinwohls vielfach verflochtener Verbund der organisatorisch weiterhin getrennten mitgliedstaatlichen Verwaltungen untereinander und mit der sich ausdifferenzierenden EG-Eigenverwaltung konstituiert.

2. Verantwortung bedeutet in diesem Mehrebenensystem einerseits die Zuordnung von Handlungszuständigkeiten und Handlungsbefugnissen zu den verschiedenen Ebenen und anderseits die demokratische und rechtsstaatliche Zurechnung und Kontrolle administrativer Handlungen des Verwaltungsverbundes. Der Begriff der Verantwortung thematisiert die Frage, wie die Kompetenzen und Befugnisse für Verwaltungshandeln in der Europäischen Union verteilt sind und wer die Ergebnisse dieses Verwaltungshandelns demokratisch und rechtsstaatlich zu vertreten hat.

3. Effizienz bezeichnet grundsätzlich das Verhältnis zwischen eingesetztem Aufwand und angestrebtem Erfolg und zielt auf eine optimale ZweckMittel-Relation. Sie begründet ein grundsätzliches Rationalitätserfordernis hoheitlicher Aufgabenerfüllung und in der Mehrebenenverwaltung konkreter die Forderungen nach organisatorischer Effizienz und Verfahrenseffizienz. Diese Forderungen sprechen für eine Verteilung der Verantwortung in der europäischen Mehrebenenverwaltung, die es der Verwaltung ermöglicht, die Verwaltungsaufgaben mit den vorhandenen Verwaltungsressourcen bestmöglich zu erledigen.

\section{Verantwortungsverteilung in der Mehrebenenverwaltung}

4. Realer Ausgangsbefund für die Verwaltung des Gemeinschaftsraums ist ein ausgeprägtes Bedürfnis nach Zusammenarbeit und Abstimmung der Verwaltungseinheiten der verschiedenen Ebenen der Mehrebenenverwal- 
tung, da nur mit dieser Zusammenarbeit und Abstimmung das europäische Gemeinwohl gemeinschaftsweit effektiv und einheitlich verwirklicht werden kann.

5. Diesem Kooperationsbedürfnis trägt das europäische Primärrecht Rechnung. Zwar beruht die Kompetenzverteilung zwischen Union und Mitgliedstaaten auch hinsichtlich der Verwaltungskompetenzen auf dem Prinzip der begrenzten Einzelermächtigung, und die Gemeinschaftsverträge weisen den Gemeinschaften keine umfassende Kompetenz zur Administration ihrer eigenen Rechtsordnung zu, sondern sehen lediglich punktuell ausdrücklich eigene gemeinschaftliche Vollzugskompetenzen vor. Die Gemeinschaftsverträge schließen eine weitergehende Gemeinschaftsverwaltung aber nicht aus, sondern übertragen dem Gemeinschaftsgesetzgeber die Entscheidung darüber, weitere Bereiche des Gemeinschaftsrechts in gemeinschaftseigenen Vollzug zu überführen oder variable Formen der Kooperation zwischen Gemeinschaft und Mitgliedstaaten oder zwischen den Mitgliedstaaten untereinander beim Vollzug des Gemeinschaftsrechts vorzuschreiben.

6. Kompetentielle Grundlage sowohl der Ausdifferenzierung der EGEigenverwaltung als auch der sekundärrechtlichen Vorschriften, die EGEigenverwaltung und Verwaltungskooperation vorsehen, sind die funktional ausgerichteten Kompetenzzuweisungen für bestimmte Gemeinschaftspolitiken. Im Rahmen vorhandener Sachkompetenzen kann der Gemeinschaftsgesetzgeber flexibel über Umfang und Art eigener Vollzugskompetenzen der EG wie über die gemeinschaftsrechtliche Festlegung von Kooperationsverfahren der Mitgliedstaaten untereinander oder mit der EG-Eigenverwaltung entscheiden. Begrenzt werden die entsprechenden Gemeinschaftskompetenzen durch das Subsidiaritätsprinzip und den Verhältnismäßigkeitsgrundsatz.

7. Damit gibt es im europäischen Primärrecht keine feste Vorrangregel für die Zuordnung der Vollzugskompetenzen zur europäischen oder mitgliedstaatlichen Ebene. Die Gemeinschaftsverfassung ermöglicht eine flexible und sachorientierte Verantwortungszuweisung innerhalb der europäischen Mehrebenenverwaltung, bei der Gesichtspunkte der Effektivität oder Effizienz des Vollzugs der Gemeinschaftsrechtsordnung für die Zuständigkeitsverteilung oder Zuständigkeitsverflechtung berücksichtigt werden können.

8. Eine vergleichbare Flexibilität ist im deutschen Bundesstaat nicht auszumachen. Hier erfolgt der Vollzug des Gemeinschaftsrechts - wie der des nationalen deutschen Rechts - im Rahmen des durch die Art. 30, 83ff. GG ausgestalteten Exekutivföderalismus grundsätzlich durch die Bundesländer. Der Bund kann den Vollzug unter den Voraussetzungen des Art. 87 Abs. 3 $G G$ auch selbst durch eigene Bundesoberbehörden oder Anstalten übernehmen, wie dies insbesondere im Bereich der landwirtschaftlichen Marktordnungen geschehen ist. Einwirkungsmöglichkeiten des Bundes auf die Vollzugstätigkeit der Länder und mögliche Arten des Zusammenwirkens oder 
der Verflechtungen beider Ebenen sind dagegen auf die in den Art. 83ff. GG vorgesehenen allgemeinen Befugnisse des Bundes bei der Verwaltung durch die Ländern begrenzt, die Entwicklung neuer Formen der Zusammenarbeit und Verflechtung außerhalb der Modelle des Grundgesetzes ist unzulässig.

\section{Formen kooperativer Verantwortungsverteilung in der europäischen Mehrebenenverwaltung}

9. Die mehrebenentypische Verflechtung von Verwaltungsverantwortlichkeiten findet sich aufgrund der festgestellten flexibleren Kompetenzverteilung in erster Linie zwischen den beiden oberen Ebenen des europäischen Mehrebenensystems, zwischen der EU und ihren Mitgliedstaaten. Auf der Grundlage entsprechender Kooperationsgesetzgebung des Gemeinschaftsgesetzgebers sind vielfältige Formen europäischer Verwaltungskooperation entstanden, die die nationalen Verwaltungen untereinander und mit der europäischen Verwaltung verschränken und sie in unterschiedliche Strukturen des verbundenen Vollzugs von Gemeinschaftsrecht einbinden. Diese Verantwortungsverflechtung, die gemeinsame oder geteilte Verantwortung für einzelne Teile, Abschnitte oder Inhalte von Verwaltungsverfahren und Verwaltungsentscheidungen, durchzieht und prägt sämtliche Formen des Verwaltungsvollzugs.

10. Beim mitgliedstaatlichen Einzelvollzug konkretisiert sie sich in Aufsichts- und Kontrollrechten der Kommission, Notifikations- und Berichtspflichten der Mitgliedstaaten, rechtlich nicht bindenden, aber faktisch effektiven Steuerungsinstrumenten der Kommission wie Informationsvermerken und Rechnungsabschlussverfahren oder in Weisungs-, Inspektions- und Kontrollbefugnissen auf sekundärrechtlicher Grundlage. Gesteigert ist die Verflechtungsintensität in gestuften Verwaltungsverfahren, bei denen die nationalen Behörden im Außenverhältnis gegenüber dem Bürger für den Vollzug des Gemeinschaftsrechts verantwortlich sind, innerhalb der Mehrebenenverwaltung jedoch ihre Entscheidungsbefugnis an die Mitwirkung der Kommission im Innenverhältnis gebunden ist.

11. Bei Referenzentscheidungen und transnationalen Verwaltungsakten erfolgt der Verwaltungsvollzug einzelstaatlich, die anderen Mitgliedstaaten sind jedoch kraft Gemeinschaftsrechts verpflichtet, die mitgliedstaatliche Entscheidung nach nur begrenzter eigener Prüfung anzuerkennen, oder ihr wird kraft Gemeinschaftsrechts gemeinschaftsweite Wirkung zuerkannt. Verweigern andere Mitgliedstaaten die Anerkennung, ist regelmäßig ein mehrstufig ausgestaltetes Divergenzbereinigungsverfahren vorgesehen, innerhalb dessen die Entscheidungskompetenz auf die Kommission übergehen kann. 
12. Beim Direktvollzug durch die EG-Eigenverwaltung greift diese bei der Entscheidungsvorbereitung oder Implementationskontrolle für Ermittlungen und Kontrollen vor Ort oder zur Vornahme wissenschaftlicher oder technischer Untersuchungen und Prüfungen auf die mitgliedstaatlichen Verwaltungen zurück. Darüber hinaus erfolgt beim Direktvollzug eine Verschränkung der europäischen und der mitgliedstaatlichen Ebene über die Komitologieverfahren.

13. Ergänzt werden diese vollzugstypabhängigen Zusammenarbeitsformen durch die alle Vollzugsvarianten des Gemeinschaftsrechts erfassenden Kooperationsformen der Netzwerkbildung und der institutionalisierten Kooperation in den Ausschüssen. Die zunehmende auch rechtliche Anerkennung der Netzwerkstruktur findet Ausdruck etwa beim Netzwerk zwischen der EU-Kommission und den nationalen Kartellbehörden oder im europäischen Regulierungsverbund nach den neuen Telekommunikationsrichtlinien.

14. In all diesen Gestaltungsformen durchziehen Kooperation und Verflechtung als Modi der Verantwortungswahrnehmung auf der Grundlage sekundärrechtlicher Vorgaben sämtliche Bereiche des Verwaltens im Verhältnis von EU und Mitgliedstaaten. Die europäische Mehrebenenverwaltung ist damit nicht auf Trennung der Verwaltungskompetenzen und Betonung der organisatorischen Eigenständigkeit von EU und Mitgliedstaaten ausgerichtet, sondern betont durch die flexiblen sekundärrechtlichen Kompetenzzuweisungen und -verschränkungen die Variabilität der Aufgabenwahrnehmung im Mehrebenensystem und zugleich die funktionale Verbundenheit und Angewiesenheit der verschiedenen Ebenen aufeinander. Klare, einheitlichen Kriterien und Baumustern folgende und durchgängig verwirklichte Verantwortungsstrukturen lassen sich in diesem System allerdings nur schwerlich erkennen.

\section{Demokratische und rechtsstaatliche Verantwortung}

15. Gerade angesichts der flexiblen und verschränkten Verteilung der Handlungszuständigkeiten und Handlungsbefugnisse innerhalb der europäischen Mehrebenenverwaltung, angesichts derer von durchgängig verwirklichten und eindeutig erkennbaren Verantwortungsstrukturen bei der Verwaltung des Gemeinschaftsraums bisher keine Rede sein kann, gewinnen die Anforderungen demokratischer und rechtsstaatlicher Zurechnung und Verantwortlichkeit besonderes Gewicht. Die europäische Verwaltung und die Verantwortungsverteilung in ihr müssen sich an den grundlegenden Anforderungen von Demokratie und Rechtsstaatlichkeit an jede Hoheitsausübung messen lassen. 
16. Aus dem Demokratieprinzip folgt insbesondere das Erfordernis hinreichender demokratischer Legitimation. Nicht nur die europäische Rechtsetzung, sondern ebenso die europäische Mehrebenenverwaltung und das europäische Verwaltungshandeln bedürfen einer ausreichenden demokratischen Legitimation im Sinne einer hinreichenden Rückbindung der Herrschaftsausübung an den Willen der europäischen Herrschaftsunterworfenen und einer Zurechenbarkeit von Verwaltungshandlungen zu bestimmten Verwaltungsträgern.

17. Für die Legitimation des Verwaltungshandelns im europäischen Verwaltungsverbund muss allerdings das traditionelle nationale repräsentativdemokratische Legitimationsmodell versagen: Es kann weder die Vielfalt der an der europäischen Verwaltung beteiligten administrativen Akteure noch deren vielfältige unterschiedliche Anteile an Verwaltungshandlungen im Verwaltungsverbund noch die angesichts dieser Vielfalt relevanten unterschiedlichen, teils verflochtenen und teils gegenläufigen Legitimationsstränge erfassen. Es wird den Schwierigkeiten der Zuordnung der verflochtenen Teile administrativen Handelns zu einzelnen Ebenen der Mehrebenenverwaltung ebenso wenig gerecht wie der qualitativ heterogenen Zusammensetzung im europäischen Verwaltungsverfahren instrumentalisierbarer Legitimationsbausteine.

18. Für die demokratische Legitimation der verflochtenen Mehrebenenverwaltung müssen zusätzliche Legitimationsbausteine akzeptiert und bei der sekundärrechtlichen Begründung und Ausgestaltung der konkreten Verantwortungsverteilung im Mehrebenensystem eingesetzt werden: die grundsätzliche strukturelle demokratische Legitimation der europäischen Mehrebenenverwaltung, Partizipation nicht nur der unmittelbar Betroffenen, sondern auch Drittbetroffener und interessierter Dritter, Transparenz und Verantwortungsklarheit sowie Effizienz der Verwaltung als output-orientierter Legitimationsansatz, weil Teil des demokratisch geforderten Verwaltungshandelns auch die bestmögliche und effektive Erledigung der übertragenen Gemeinwohlaufgaben ist.

19. Darüber hinaus muss die Verantwortungszuweisung in der europäischen Verwaltung rechtsstaatlichen Anforderungen genügen. Zentrale Anforderungen der Rechtsstaatlichkeit sind insoweit zumindest Verantwortungsklarheit, effektiver Rechtsschutz und eindeutige Haftung. Hier ist rechtsstaatlich zumindest geboten, mit den materiellen Kooperationsvorschriften sachgebiets- und verfahrensspezifisch eindeutige Rechtsschutz- und Haftungsregelungen aufzustellen und die Betroffenen des kooperativen Verwaltungshandelns hierüber ausführlich zu belehren und zu informieren. 


\section{Perspektiven}

20. Die Aufgaben und Befugnisse der Verwaltung sind im europäischen Mehrebenensystem weder der EU noch ihren Mitgliedstaaten starr und eindeutig zugewiesen. Vielmehr übertragen die funktional ausgerichteten Gemeinschaftskompetenzen dem Gemeinschaftsgesetzgeber die Aufgabe, je nach sachgebietsspezifischer Notwendigkeit in den Grenzen des Subsidiaritätsprinzips und des Verhältnismäßigkeitsgrundsatzes über die Zuweisung der Verwaltungsverantwortung zu entscheiden. Zulässig und unter Effizienzgesichtspunkten sinnvoll ist dabei auch die gemeinsame arbeitsteilige Wahrnehmung von Verwaltungsverantwortung durch Zusammenarbeit von Verwaltungseinrichtungen der verschiedenen Ebenen oder innerhalb einer Ebene.

21. Bei der durch diese flexible Verantwortungsstruktur ermöglichten sekundärrechtlichen Verantwortungsverteilung muss der Gemeinschaftsgesetzgeber die demokratischen und rechtsstaatlichen Anforderungen der europäischen Verfassung an den europäischen Verwaltungsverbund berücksichtigen. Aus diesen folgen Optimierungsgebote für die demokratische und rechtsstaatliche Ausgestaltung der Institutionen und Verfahren der Verantwortungswahrnehmung: Verantwortung ist im europäischen Mehrebenensystem so zu verteilen, dass sie im strukturell grundsätzlich demokratisch legitimierten europäischen Verwaltungsgefüge klar und eindeutig zugeordnet ist, dass Transparenz über Zuständigkeiten und Verfahren besteht, dass die Partizipation Betroffener und Interessierter am Verfahren ermöglicht wird und dass im Rahmen dieser Vorgaben die Verwaltungsaufgaben effizient wahrgenommen werden können.

22. Dieser komplexe Optimierungsauftrag an den europäischen Gesetzgeber stellt die erforderliche demokratisch-rechtsstaatliche Einhegung der großen Flexibilität der primärrechtlichen Verantwortungsverteilung zwischen den oberen Ebenen der europäischen Mehrebenenverwaltung sicher. Seine Erfüllung könnte erleichtert werden durch den Versuch der sekundärrechtlichen Kodifikation einer Typik von Kooperationsformen, die der europäische Gesetzgeber bereichsspezifisch je nach den festgestellten Kooperationsbedürfnissen für die sachlich angemessene Verantwortungszuweisung innerhalb der europäischen Mehrebenenverwaltung einsetzen könnte. Bereits der Versuch einer solchen Kodifikation strukturierter Typen von Verwaltungsverantwortung und Verwaltungskooperation, eines demokratisch und rechtsstaatlich ausgestalteten allgemeinen Teils des europäischen Verwaltungskooperationsrechts mit Regelungen zu Verantwortlichkeiten, Beteiligungsrechten, Informations-, Belehrungs- und Begründungspflichten sowie zu Rechtsschutz und Haftung bei den unterschiedlichen Modi der Verantwortungswahrnehmung im europäischen Verwaltungsverbund könnte auf 
europäischer und auf nationaler Ebene jedenfalls das Bewusstsein um die demokratischen und rechtsstaatlichen Anforderungen der Verwaltungskooperation in der Mehrebenenverwaltung deutlich schärfen.

23. Unabhängig hiervon darf bereits gegenwärtig trotz aller tatsächlichen Notwendigkeit von Verwaltungskooperation und trotz der großen rechtlichen Flexibilität der europäischen Kompetenzordnung nicht Effizienz allein als Maßstab für Verantwortung im europäischen Mehrebenensystem angesehen werden. Nur eingebunden in die grundlegenden demokratischen und rechtsstaatlichen Anforderungen des europäischen Verfassungsverbundes können auch Effizienzgesichtspunkte Bedeutung für die Verantwortungsverteilung in der europäischen Mehrebenenverwaltung erlangen. 
Zweiter Beratungsgegenstand:

\section{Verantwortung und Effizienz in der Mehrebenenverwaltung}

2. Bericht von Professor Dr. Thomas Groß, Gießen*

Inhalt

Seite

I. Die polyzentrische Struktur der Mehrebenenverwaltung . . 153

II. Die Regeln der Aufgabenverteilung . . . . . . . . . . . . . . 157

1. Formen der Aufgabenverteilung . . . . . . . . . 157

2. Allgemeine Rechtsgrundsätze der Aufgabenverteilung . . 159

III. Die Ausgestaltung der Ebenenkoordination . . . . . . . . 163

1. Die Typen der vertikalen Koordination . . . . . . . . 163

2. Die Durchsetzungsmechanismen . . . . . . . . . 167

IV. Die Schaffung von Legitimation als Gestaltungsaufgabe . . . 169

1. Die Verantwortungsverteilung im Mehrebenensystem . . 169

2. Das institutionelle Legitimationsarrangement . . . . . . . 171

V. Fazit . . . . . . . . . . . . . . . 176

* Für wertvolle Hinweise und Kritik danke ich Gabriele Britz, Pascale Cancik, Timo Hebeler, Hans Christian Röhl und Manon Schwarze. 
Die Leitbegriffe Verantwortung und Effizienz thematisieren das Grundproblem jeder, insbesondere einer mehrstufigen Verwaltung: Wie kann die Verteilung von administrativen Aufgaben auf verschiedene Ebenen so organisiert werden, dass einerseits ein wirksamer demokratischer $\mathrm{Zu}$ rechnungszusammenhang besteht, andererseits die Aufgaben möglichst erfolgreich erfüllt werden? Besonders kritisch wird diese Frage im Hinblick auf die Verflechtung zwischen der europäischen Verwaltung und den nationalen Verwaltungen diskutiert. ${ }^{1}$ Sie stellt sich aber auch in jedem Bundesstaat. ${ }^{2}$ Welche Regeln gewährleisten, dass die selbständigen Akteure mehrerer Ebenen in eine funktionsfähige gemeinsame Ordnung eingebunden werden? ${ }^{3}$

Die Fragestellung erfordert zunächst eine kurze begriffliche und methodische Einführung (I.). In einem zweiten Schritt ist zu klären, nach welchen Mustern die Aufgaben zwischen den Ebenen verteilt werden (II.). Drittens werden die einzelnen Koordinationsmechanismen der Mehrebenenverwaltung untersucht (III.). Und schließlich ist viertens zu diskutieren, wie die demokratische Verantwortung in einem solchen mehrfach gestuften System gesichert werden kann (IV.).

\section{Die polyzentrische Struktur der Mehrebenenverwaltung}

Aufgrund der fehlenden politischen Klärung der Finalität der europäischen Integration ist die staatstheoretische Einordnung der Union als föderales Gebilde bis heute umstritten. ${ }^{4}$ Neu geschaffene Kategorien wie

1 A. Benz in: Jachtenfuchs/Kohler-Koch (Hrsg.) Europäische Integration, 2. Aufl. 2003, 317 (339); E. Schmidt-Aßmann in: Hoffmann-Riem/Schmidt-Aßmann/Voßkuhle (Hrsg.) Grundlagen des Verwaltungsrechts (GVwR), Bd. I, 2006, § 5 Rn. 25 ff.; sogar von einer „Fusionsbürokratie“ sprechen M. Bach ZfS 1992, 16 (24), und W. Wessels Die Öffnung des Staates, 2000, $122 \mathrm{ff}$.

2 Vgl. zum GG knapp H. Wißmann GVwR I, § 15 Rn. 26; interessanterweise findet sich der Begriff des Mehrebenensystems schon früh in einer verwaltungswissenschaftlichen Untersuchung von Problemen der deutschen föderativen Ordnung, vgl. D. Schimanke VerwArch 68 (1977), 361 (369).

${ }^{3}$ C. Franchini, in: Chiti/Greco (Hrsg.), Trattato di Diritto amministrativo europeo, Parte generale, 1997, 467 (472): „ordinamento integrato“; M. Jestaedt GVwR I, § 14 Rn. 54: „kohärente Entscheidungs- und Wirkeinheit“.

${ }^{4}$ So z.B. M. Schweitzer VVDStRL 53 (1994), 48 (49); U. Everling FS Badura, 2004, 1053 (1056); s.a. R. Wahl in: H. Dreier (Hrsg.) Rechts- und staatstheoretische Schlüsselbegriffe: Legitimität - Repräsentation - Freiheit, 2005, 113 (126ff.); Ch. Schönberger Unionsbürger, 2005, $6 \mathrm{ff}$;; ausführlich auch zur geschichtlichen Entwicklung M. Burgess Federalism and European Union: the Building of Europe, 1950-2000, 2000. 
Supranationalität, ${ }^{5}$ Transnationalität, ${ }^{6}$ Staatenverbund ${ }^{7}$ zeugen von dieser Debatte. Wie auch die Übergangsbeschreibung als Gebilde sui generis ${ }^{8}$ sind diese Begriffe aber für die konkrete Analyse der Verwaltungsbeziehungen wenig hilfreich. ${ }^{9}$ Die Kommission ist bei ihren administrativen Funktionen in vielfältiger Weise mit den nationalen Verwaltungen verknüpft, insbesondere durch das Ausschusswesen und gestufte Verwaltungsverfahren, in denen eine enge Zusammenarbeit erfolgt. ${ }^{10}$ Dies gilt ebenso für die Agenturen, ${ }^{11}$ eine nur lose mit der Kommission verkoppelte neue Schicht europäischer Verwaltung. ${ }^{12}$

Um die Fixierung auf den Nationalstaat als zentraler Kategorie zur Strukturierung der Herrschaftsbeziehungen $\mathrm{zu}$ relativieren und der praktischen Bedeutung der europäischen Verwaltung gerecht zu werden, sind verschiedene neue Begriffe eingeführt worden. Der v.a. in der Politikwissenschaft prominente Terminus „Mehrebenensystem“ hat den Charme, gestufte Formen der Herrschaftsausübung zu erfassen, ohne eine vorgegebene Präponderanz einer bestimmten Ebene anzuerkennen, bleibt aber sehr allgemein. Speziell zur Charakterisierung der europäischen Verwaltungskooperation wird in den letzten Jahren oft der

${ }^{5}$ Dazu bereits G. Erler VVDStRL 18 (1960), 7 (15ff.); aus der jüngeren Literatur z.B. A. v. Bogdandy Supranationaler Föderalismus als Wirklichkeit und Idee einer neuen Herrschaftsform, 1999, 61 ff.; S. Oeter in: v. Bogdandy (Hrsg.) Europäisches Verfassungsrecht, 2003, 59 (63ff.).

${ }^{6}$ K. Nicolaidis in: ders./Howse (Hrsg.) The Federal Vision - Legitimacy and Levels of Government in the United States and the European Union, 2001, 439 (467); A. Böhmer Die Europäische Union im Lichte der Reichsverfassung von 1871, 1999, $144 \mathrm{ff}$.

7 P. Kirchhof in: Isensee/Kirchhof (Hrsg.) Handbuch des Staatsrechts (HStR), Bd. VII, 1992, § 183 Rn. 54; BVerfGE 89, 155 (181).

8 L. Hooghe/G. Marks Multi-Level Governance and European Integration, 2001, 35.

9 M. Jachtenfuchs/B. Kohler-Koch in: dies. (Fn. 1), 11 (18); Ch. Schönberger AöR 129 (2004), 81 (83f.).

10 Grundlegend E. Schmidt-Aßmann EuR 1996, 270; G. Sydow Verwaltungskooperation in der Europäischen Union, 2004, 3 ff.; s. a. J. Schwarze Europäisches Verwaltungsrecht, 2. Aufl. 2005, CI ff.

11 Vgl. Th. Groß EuR 2005, 54.

12 Vgl. den Überblick bei J. Gundel, in: Schulze/Zuleeg (Hrsg.), Europarecht, 2006, $\S 3$ Rn. 25 ff.; s.a. D. Fischer-Appelt Agenturen der Europäischen Gemeinschaft, 1999, $218 \mathrm{ff}$;; Th. Groß Das Kollegialprinzip in der Verwaltungsorganisation, 1999, $337 \mathrm{ff}$;; D. Riedel Die Gemeinschaftszulassung für Luftgerät, 2006, $184 \mathrm{ff}$; H. Schulze-Fielitz in: Erbguth/Masing (Hrsg.), Verwaltung unter dem Einfluss des Europarechts, 2006, 91 (97ff.); zu den sonstigen Formen der Externalisierung vgl. W. Schenk Strukturen und Rechtsfragen der gemeinschaftlichen Leistungsverwaltung, 2006, 176ff.; kritisch G. Lübbe-Wolff VVDStRL 60 (2001), 246 (269ff.), auch zur Übertragung von Aufgaben der Subventionsverwaltung auf Private. 
Begriff des Netzwerkes verwendet. ${ }^{13}$ So richtig die Beobachtung ist, dass horizontalen Formen der Koordination in der Union eine wesentliche Rolle zukommt, sollte man doch die hierarchischen Elemente nicht übersehen, die auch im Verhältnis der europäischen Verwaltung zu den nationalen Behörden bestehen. ${ }^{14}$ Dass es um eine komplexe und keineswegs für alle Gebiete einheitliche Kombination verschiedener Instrumente der Organisation von Kooperation geht, verdeutlicht besser der Begriff des Verwaltungsverbundes. ${ }^{15}$

Alle drei terminologischen Angebote weisen also auf zutreffende Aspekte der europäisierten Verwaltung hin. Für die Lösung konkreter Rechtsfragen im Verhältnis zwischen den Ebenen leisten sie jedoch keinen Beitrag. ${ }^{16}$ Die Problematik der Verflechtung mehrerer Ebenen ist aber kein neues Phänomen, sondern ein klassisches Thema des Bundesstaates. ${ }^{17}$ Deshalb soll hier die Beobachtung, dass die europäische Integration durch einen Prozess der administrativen Föderalisierung gekennzeichnet ist, ${ }^{18}$ wieder aufgegriffen werden. Es gilt, den Erfahrungsschatz der bundesstaatlichen Dogmatik zu nutzen, um das Verhältnis zwischen den Organen der Gemeinschaft und den Mitgliedstaaten zu analysieren. Bereits in den ersten juristischen Analysen des SchumanPlans aus dem Jahr 1951 wurde darauf hingewiesen, dass er mit organisatorischen Mitteln arbeite, die bisher nur dem Zusammenschluss

13 A. Gil Ibáñez The Administrative Supervision and Enforcement of EC Law: Powers, Procedures and Limits, 1999, $291 \mathrm{ff}$; H. Schmitt v. Sydow in: Magiera/Sommermann (Hrsg.) Verwaltung und Governance im Mehrebenensystem der Europäischen Union, 2002, 171 (180f.); L. Azoulay in: Joerges/Dehousse (Hrsg.) Good Governance in Europe's Integrated Market, 2002, S. 109 (126); M. Jachtenfuchs/B. Kohler-Koch (Fn. 9), 25; M. Ruffert DV 36 (2003), 293 (311ff.); Th. Vesting VVDStRL 63 (2004), 41 (56ff.); G. Sydow (Fn. 10), 78 ff.; M. Bach in: Schuppert/Pernice/Haltern (Hrsg.) Europawissenschaft, 2005, 575 (596ff.); vgl. auch die Erwähnung eines „Netzes der Kartellbehörden“ in Erwägungsgrund 15 Kartell-VO (EG) 1/2003, und Erwägungsgrund 14 Fusionskontroll-VO (EG) 139/2004.

14 Pointiert T. Börzel in: Schuppert/Pernice/Haltern (Fn. 13), 613 (625).

$15 \mathrm{Vgl}$. E. Schmidt-Aßmann, in: ders./Schöndorf-Haubold (Hrsg.) Der Europäische Verwaltungsverbund, 2005, 1 (7); J. Hofmann Rechtsschutz und Haftung im Mehrebenenverbund, 2004, 27 f.; G. Britz EuR 2006, 46 (47), weist zu Recht darauf hin, dass dies ein „deskriptiv-analytischer Begriff“ ist.

${ }_{16}$ Zutreffend M. Jestaedt GVwR I, § 14 Rn. 18; ebenso zum Begriff des Netzwerkes W. Weiß DV 38 (2005), 517 (521); F. Wettner Die Amtshilfe im Europäischen Verwaltungsrecht, 2005, 290.

17 Zur Parallele vgl. z.B. Th. Hueglin in: Kohler-Koch/Eising (Hrsg.) The Transformation of Governance in the European Union, 1999, 249 (250f.); E. Grande in: ders. (Hrsg.) Wie problemlösungsfähig ist die EU?, 2000, 11 (16).

18 Th. Groß JZ 1994, 596 (605). 
zu einem Bundesstaat gedient haben, ${ }^{19}$ und deshalb der Begriff des „bundesstaatsähnlichen Gebildes“ verwendet. ${ }^{20}$ Auch außerhalb der deutschen Debatte werden inzwischen vielfach Tendenzen zu einer Föderalisierung der Gemeinschaften diskutiert. ${ }^{21}$

Durch die Integration eines Bundesstaates in die Europäische Gemeinschaft entsteht ein doppelter Föderalismus, ${ }^{22}$ in dem drei Ebenen der Gesetzgebung und drei Ebenen der Verwaltung - unter Einbeziehung der kommunalen Ebene sogar vier - miteinander kombiniert werden. Allerdings gibt es keine einheitlichen Regeln für die Koordination dieses Mehrebenensystems, da die jeweilige konstitutionelle Ordnung meist nur das Verhältnis zur nächstunteren Ebene erfasst. Erschwert wird die Analyse zudem dadurch, dass im Primärrecht der Union allgemeine Vorgaben für ihr Verhältnis zu den nationalen Verwaltungen fehlen, da ihre Kompetenzordnung nicht wie in den bundesstaatlichen Verfassungen funktionsbezogen ist. ${ }^{23}$

Deshalb sollen hier mit einem vertikalen und horizontalen Rechtsvergleich funktional parallele Erscheinungen der Mehrebenenkoordination aufgezeigt werden. Bei einer abstrakten Analyse weist das Verhältnis zwischen Gemeinschaft und Mitgliedstaaten viele strukturelle Ähnlichkeiten mit dem Bund-Länder-Verhältnis, auch in Österreich und der Schweiz, sowie dem Verhältnis zwischen Staat und Kommunen auf. Auf allen vier Ebenen bestehen politisch selbständig legitimierte Verwaltungen, deren Koordination durch verfassungsrechtliche und gesetzliche Regelungen organisiert wird, während gleichzeitig aber auch die Autonomie der unteren Ebenen respektiert werden muss. In der Summe bil-

19 H. Mosler ZaöRV Bd. XIV (1951/52), 1 (8).

20 C. F. Ophüls NJW 1951, 289 (289); ähnlich H.-J. Schlochauer JZ 1951, 289 (289); W. Thieme VVDStRL 18 (1960), 50 (68); s. a. D. Vignes La Communauté européenne du Charbon et de l'Acier, 1956, 12: „fédéralisme fonctionnel“; ablehnend P. Badura VVDStRL 23 (1966), 34 (49f.).

21 Vgl. z. B. P. Craig Oxford Journal of Legal Studies 12 (1992), 453 (458); K. Lenaerts ELR 1993, 23 (28); M. Chiti in: ders./Greco (Fn. 3), 167 (167); F. Delpérée Le fédéralisme en Europe, 1999, 79; Ph. Manin Les Commmunautés européennes, L'Union européenne, 5. Aufl. 1999, 64; A. Gil Ibáñez (Fn. 13), 251; S. Bartolini Restructuring Europe, 2005, 141.

22 Vgl. H. Bülck VVDStRL 21 (1964), 1 (15); s. a. K. Bretz Föderalismus und Regionalismus in Deutschland, Spanien und der Europäischen Union, 2005, 240; das Problem thematisierte schon H. Kelsen ZÖR Bd. VI (1927), 329 (331), der bei einem Beitritt des österreichischen Bundesstaates zum Deutschen Reich „heillose organisationstechnische Komplikationen" befürchtete.

${ }^{23}$ A. David Inspektionen im Europäischen Verwaltungsrecht, 2003, 167; H. Ch. Röhl DVB1. 2006, 1070 (1072). 
den sie ein polyzentrisches System, in dem kein Akteur eine dominante Rolle spielen kann. ${ }^{24}$ Der Begriff der Koordination soll deutlich machen, dass es nicht um eine zu stark vereinfachende Dichotomie zwischen Hierarchie und Netzwerk geht, sondern komplexere Regelungsstrukturen $\mathrm{zu}$ analysieren sind. ${ }^{25}$

\section{Die Regeln der Aufgabenverteilung}

\section{Formen der Aufgabenverteilung}

Sowohl in der Bundesrepublik und der Schweiz wie auch in der Europäischen Union ist die zentralisierte Ausführung der Gesetze durch Behörden der Bundesverwaltung bzw. der Gemeinschaft die Ausnahme. Während das Grundgesetz (Art. 83 GG) und die Schweizer Bundesverfassung (Art. 46 Abs. 1 BV) den Vollzug durch die Gliedstaaten als Regelfall vorgeben, erfolgt im österreichischen Bundes-Verfassungsgesetz (Art. $10 \mathrm{ff}$. B-VG) eine materienspezifische Verteilung der Vollziehungsaufgaben auf Bund und Länder, die keinen normativen Vorrang der mittelbaren Bundesverwaltung, d.h. der Ausführung durch die Landesregierungen, kennt. ${ }^{26}$ Keiner dieser Bundesstaaten folgt einem strikten Trennungssystem, wie es die US-amerikanische Verfassung vorsieht. ${ }^{27}$

Die Kommission hat nach dem primären Gemeinschaftsrecht nur wenige eigene Zuständigkeiten wie etwa bei der Beihilfenaufsicht. ${ }^{28}$ Daraus ist ein grundsätzlicher Vorrang des mitgliedstaatlichen Vollzugs abgeleitet worden. ${ }^{29}$ Dies verkennt jedoch die Struktur der gemeinschaftsrechtlichen Kompetenznormen. Ihre finale Ausgestaltung orientiert sich nicht wie das Grundgesetz am Schema der Gewaltenteilung, sondern überlässt es regelmäßig dem europäischen Gesetzgeber, die Maßnahmen zur Erreichung der vorgegebenen Ziele festzulegen. Deshalb ist die Übertragung von Verwaltungskompetenzen auf die Kommission wie auch die

24 Vgl. A. Peters Elemente einer Theorie der Verfassung Europas, 2001, $215 \mathrm{ff}$. m.w.N.

25 Ähnlich ist der Ansatz des allerdings sehr heterogen verwendeten Konzeptes der Governance, vgl. z.B. R. Eising/B. Kohler-Koch in: Kohler-Koch/Eising (Fn. 17), 3 (5); A. Voßkuhle GVwR I, § 1, 21, $68 \mathrm{ff}$; ausführlich jetzt C. Franzius VerwArch 97 (2006), 186; für die EU Ch. Möllers CMLR 2006, 313.

${ }^{26}$ Dazu ausführlich K. Weber Die mittelbare Bundesverwaltung, 1987, $99 \mathrm{ff}$.

${ }^{27}$ Dazu V. Mehde Wettbewerb zwischen Staaten, 2005, $362 \mathrm{ff}$.

${ }_{28}$ Zur engen Auslegung der Zuständigkeit des Rates nach Art. 88 Abs. 2 UAbs. 3 vgl. EuGH, EuZW 2006, 497 (498).

${ }^{29}$ R. Streinz HStR VII, 1992, § 182 Rn. 4; S. Hegels EG-Eigenverwaltungsrecht und Gemeinschaftsverwaltungsrecht, 2001, 28; H. Wißmann GVwR I, § 15 Rn. 22. 
Gründung von Agenturen mit administrativen Aufgaben auf der Grundlage geeigneter Sachkompetenzen oder hilfsweise des Art. 308 EGV zulässig. ${ }^{30}$

Neben dem dezentralen Gesetzesvollzug und der reinen Zentralverwaltung gibt es aber auch zwei Mischformen: die Zentralverwaltung unter Mitwirkung von Vertretern der dezentralen Ebene und die gemeinsame Verwaltung. Besonders ausgeprägt ist die erste Variante auf der europäischen Ebene. In vielen Bereichen sind Entscheidungskompetenzen der Kommission durch die obligatorische Einbeziehung von Ausschüssen, die mit nationalen Regierungsvertretern besetzt sind, relativiert. Neben diesen Komitologie-Verfahren ${ }^{31}$ gibt es vielfältige beratende Fachausschüsse und die Verwaltungsräte der Agenturen, in denen regelmäßig die Mitgliedstaaten vertreten sind, sowie neuerdings in mehreren Bereichen Ausschüsse, in denen nationale Regulierungsbehörden mit der Kommission zusammenarbeiten. ${ }^{32}$

In der deutschen Verwaltungsorganisation sind solche Formen der Beteiligung von Vertretern der dezentralen Ebene dagegen selten. Beispiele auf Bundesebene sind die drei Beiräte der Bundesnetzagentur ${ }^{33}$ oder die von den Landesregierungen ernannten Beisitzer der Bundesprüfstelle für jugendgefährdende Medien (§ 19 Abs. $1 \mathrm{JuSchG).} \mathrm{Auch}$ eine über die Spitzenverbände vermittelte Mitwirkung der Kommunen in Gremien auf Landesebene kommt gelegentlich vor. ${ }^{34}$

In Teilbereichen gibt es sogar eine weitergehende Verflechtung zwischen zentraler und dezentraler Ebene. In der Verwaltung der Strukturfonds der Europäischen Gemeinschaft erfolgt eine sehr enge Kooperation über gemischte Ausschüsse und gestufte Planungsverfahren, die als

${ }^{30}$ H. D. Jarass AöR 121 (1996) 173 (181ff.); G. Sydow (Fn. 10), 43 f.; D. Riedel (Fn. 12), $241 \mathrm{f}$; restriktiver u.a. M. Klepper Vollzugskompetenzen der Europäischen Gemeinschaft aus abgeleitetem Recht, 2001, $45 \mathrm{ff}$.; M. Winkelmüller Verwaltungskooperation bei der Wirtschaftsaufsicht im EG-Binnenmarkt, 2002, $145 \mathrm{ff}$.; die Heranziehung von Art. 95 EGV billigt EuGH, DVB1. 2006, 835.

${ }^{31}$ Grundlage ist der Beschluss 1999/468/EG v. 28. 6. 1999; zur beachtlichen praktischen Relevanz der Ausschussberatungen vgl. G. Schäfer in: Andemas/Türk (Hrsg.) Delegated Legislation and the Role of Committees in the EC, 2000, 3 (20).

32 Vgl. zum Energierecht K. Herzmann ZNER 2005, 216; F. Arndt DV 39 (2006), 100 (109ff.); zum TK-Recht K.-H. Ladeur/Ch. Möllers DVB1. 2005, 525 (526ff.); zum Kartellrecht J. Schwarze/A. Weitbrecht Grundzüge des europäischen Kartellverfahrensrechts, 2004, $169 \mathrm{ff}$.

33 Dazu kritisch Ch. Schmidt NVwZ 2006, 907 (909).

34 Beispiele in Hessen sind der Denkmalrat ( 5 DenkmalschutzG), der Landeskrankenhausausschuss ( $§ 21$ II HKHG) und der Landesbeirat für den Rettungsdienst ( 24 HRDG). 
„gemeinsame Verwaltung“ bezeichnet werden kann. ${ }^{35}$ Dem ähneln im deutschen Bundesstaat die Gemeinschaftsaufgaben (Art. 91a, 91b GG), denn in den gemeinsam von Bund und Ländern besetzten Planungsgremien ist ein „administratives Kondominium“ entstanden. ${ }^{36}$ In Österreich können durch Vereinbarung zwischen Bund und Ländern gemeinsame Einrichtungen gegründet werden (Art. 15a B-VG), ${ }^{37}$ die auch in der Schweiz in allerdings sehr seltenen Fällen bestehen. ${ }^{38}$ Auf der subnationalen Ebene gibt es ebenfalls Beispiele einer ebenenübergreifenden institutionalisierten Zusammenarbeit wie bei der Mitwirkung kommunaler Vertreter an der Regionalplanung ${ }^{39}$ oder der wohl misslungenen Konstruktion der Arbeitsgemeinschaften zwischen der Bundesagentur für Arbeit und den kommunalen Trägern (§ 44b SGB II). ${ }^{40}$

\section{Allgemeine Rechtsgrundsätze der Aufgabenverteilung}

Die vier möglichen Optionen der Aufgabenverteilung werfen die Frage nach übergreifenden Rechtsgrundsätzen für ihre Auswahl auf. Zum einen geht es um die Anwendung von Subsidiaritätsklauseln. Zum anderen ist zu klären, unter welchen Voraussetzungen Formen der Mischverwaltung zulässig sind.

Das Subsidiaritätsprinzip (Art. 5 Abs. 2 EGV) ist auf den administrativen Vollzug des Gemeinschaftsrechts ohne weiteres anwendbar. ${ }^{41} \mathrm{Al}$ lerdings haben sich die Erwartungen an seine begrenzende Wirkung nicht erfüllt, da seine Formulierung dem Gemeinschaftsgesetzgeber einen politischen Einschätzungsspielraum bezüglich der Notwendigkeit

${ }^{35}$ B. Schöndorf-Haubold Die Strukturfonds der Europäischen Gemeinschaft, 2005, 38 m.w.N.; H. Ch. Röhl DVB1. 2006, 1070 (1075); s.a. H.-H. Trute GVwR I, § 6 Rn. 110: „geteilte Verwaltung“.

36 U. Volkmann in: v. Mangoldt/Klein/Starck, GG, Bd. 3, 5. Aufl. 2005, Art. 91a Rn. 7; dazu näher W. Blümel HStR IV, 1990, § 101 Rn. $124 \mathrm{ff}$.

37 Th. Öhlinger Verträge im Bundesstaat, 1978, 35, bezeichnet sie als ,partielle Durchbrechung des strikten Trennungsprinzips der Kompetenzverteilung“; vgl. z. B. die durch Vereinbarung (BGB1. 2005/73) geschaffene Bundesgesundheitskommission, in der neben dem Bund auch die Länder und Interessenvertreter der Städte und Gemeinden sowie verschiedener Verbände vertreten sind.

$38 \mathrm{Vgl}$. U. Häfelin/W. Haller Schweizerisches Bundesstaatsrecht, 5. Aufl. 2001, Rn. 1262.

$39 \mathrm{Vgl}$. zu den verschiedenen Typen H.-J. Koch/R. Hendler Baurecht, Raumordnungsund Landesplanungsrecht, 4. Aufl. 2004, 84 ff.; zur Einordnung als staatlich-kommunales Kondominium vgl. E. Schmidt-Aßmann AöR 101 (1976), 520 (536).

40 Vgl. H.-G. Henneke DÖV 2006, 726.

${ }^{41}$ S. Kadelbach Allgemeines Verwaltungsrecht unter europäischem Einfluß, 1999, 112; G. Sydow (Fn. 10), 46; G. Winter EuR 2005, 255 (261). 
einer Zentralisierung von Verwaltungskompetenzen eröffnet. ${ }^{42}$ Letzteres ist insbesondere bei großen Disparitäten im Vollzug des Gemeinschaftsrechts anzunehmen, ${ }^{43}$ aber auch bei einer Überforderung einzelner Mitgliedstaaten, wie etwa im Fall der Grenzschutzagentur, ${ }^{44}$ die zur Unterstützung v.a. der Mittelmeeranrainer geschaffen wurde.

Ein dem Subsidiaritätsprinzip ähnliches Aufgabenverteilungsprinzip zugunsten der Gemeinden ergibt sich aus der Selbstverwaltungsgarantie (Art. 28 Abs. 2 S. 1 GG). Der Entzug von Aufgaben mit einem relevanten örtlichen Charakter muss durch Gründe des Gemeininteresses gerechtfertigt werden, wofür eine größere Wirtschaftlichkeit der Aufgabenerledigung nicht ausreicht. ${ }^{45}$ Ein unbedingter Vorrang der möglichst weitgehenden Betroffenenpartizipation lässt sich daraus allerdings nicht ableiten. ${ }^{46}$ Letztlich wird die gesetzgeberische Entscheidung nur auf ihre Vertretbarkeit überprüft. ${ }^{47}$

Im bundesstaatlichen Verhältnis ist die Rechtslage uneinheitlich. In der Schweiz gibt es zwar ein Subsidiaritätsprinzip für die „Umsetzung“ des Bundesrechts durch die Kantone (Art. 46 Abs. 2 BV), doch bedarf es näherer Ausgestaltung durch den Bundesgesetzgeber. ${ }^{48}$ In Österreich fehlt eine entsprechende allgemeine Vorschrift. Die Verwaltungskompetenz der deutschen Länder ist partiell dadurch geschützt, dass für die Errichtung von Mittel- und Unterbehörden des Bundes neben der Zustimmungspflicht das Erfordernis des dringenden Bedürfnisses vorgesehen ist (Art. 87 Abs. 3 S. 2 GG), das jedoch nur eingeschränkt justiziabel ist. ${ }^{49}$ Gleiches gilt für die neue Beschränkung des bundesgesetzlichen Zugriffs auf Organisations- und Verfahrensfragen der Länderverwaltung (Art. 84 Abs. 1 S. 5 GG). ${ }^{50}$ Dagegen fehlt für die Gründung von Bundesoberbehörden jede materielle oder prozedurale Begrenzung des Bundesgesetzgebers (Art. 87 Abs. 3 S. 1 GG). Deshalb wurde vertreten,

42 G. Lienbacher in: Schwarze (Hrsg.) EU-Kommentar, 2000, Art. 5 EGV Rn. 31; R. Bieber/A. Epiney/M. Haag Die Europäische Union, 6. Aufl. 2005, § 3 Rn. 33; kritisch zur Rechtsprechung des EuGH S. Albin NVwZ 2006, 629.

${ }^{43}$ M. Klepper (Fn. 30), $103 \mathrm{f}$.

44 VO (EG) Nr. 2007/2004; dazu S. Jahn Die Polizei 2006, 207.

45 BVerfGE 79, 127 (147ff.). Deutlicher ist die textliche Grundlage in einigen Landesverfassungen, z. B. Art. 137 Abs. 1 LV Hessen, Art. 71 Abs. 2 LV Baden-Württemberg.

46 BVerfGE 110, 370 (401).

47 BVerfGE 79, 127 (154); 110, 370 (400); F. Schoch VerwArch 81 (1990), 18 (34).

48 U. Thalmann in: Fleiner/Forster/Misic/Thalmann (Hrsg.) Die neue schweizerische Bundesverfassung, 2000, 149 (165); R. Rhinow Die Bundesverfassung 2000, 2000, 85.

${ }^{49}$ So wohl auch H.-H. Trute in: v. Mangoldt/Klein/Starck (Fn. 36), Art. 87 Rn. 112.

${ }^{50}$ Zur Diskussion in der Föderalismus-Kommission vgl. F. Kirchhof ZG 2004, 209 (219f.). 
dass aufgrund eines allgemeinen Prinzips auch hierfür ein Bedürfnis des Bundes erforderlich sei. ${ }^{51}$ Angesichts der Regelungsintensität des Grundgesetzes für das Bund-Länder-Verhältnis ist für eine solche Verallgemeinerung jedoch kein Platz. Jenseits der expliziten Beschränkungen des Bundes lässt sich dem Grundgesetz kein ungeschriebener Grundsatz entnehmen, wonach die dezentrale Ebene vorzuziehen und die Zentralisierung von Verwaltungsaufgaben deshalb begründungsbedürftig ist. 52

Auch die Versuche der ökonomischen Föderalismustheorie, einen generellen Vorrang der niedrigeren Ebene zu begründen, überzeugen nicht. Sie bevorzugen ohne hinreichende empirische Grundlage die Möglichkeit der kleineren Einheiten, auf lokale Präferenzen einzugehen, während die Organisationskosten dezentraler Aufgabenerfüllung als sekundär dargestellt werden.53 Für die notwendige Abwägung zwischen den Vor- und Nachteilen der föderalisierten Verwaltung gibt es aber keine wissenschaftlich objektiv feststellbaren Kriterien, da die drei zentralen Faktoren der Qualität der Aufgabenerfüllung, ihrer Wirtschaftlichkeit und der Intensität der Bürgerpartizipation inkommensurable Größen sind. ${ }^{54}$ Diese Erkenntnis bestärkt die Auslegung aller Subsidiaritätsklauseln, wonach die Entscheidung, ab wann die Disparitäten bei einer dezentralen Aufgabenerfüllung als intolerabel anzusehen sind, so dass die höhere Ebene den Vollzug an sich zieht, vorrangig nach politischen Kriterien zu treffen ist.

Die unzähligen Ausschüsse, in denen Vertreter der Kommission und der Mitgliedstaaten kooperieren, werden aus deutscher Sicht oft als

51 Vgl. H. Kalkbrenner JZ 1963, 210 (213); G. Britz DVB1. 1998, 1167 (1173); a.A. P. Lerche in: Maunz/Dürig Grundgesetz, Art. 87 Rn. 179; G. Hermes in: Dreier (Hrsg.) Grundgesetz, Bd. 3, 2000, Art. 87 Rn. 67, betont die Korrekturfunktion der weiten Bundeskompetenz.

52 S. U. Pieper Subsidiarität, 1994, 56, weist zu Recht darauf hin, dass es keinen notwendigen Zusammenhang zwischen Föderalismus und Subsidiarität gibt; ebenso bereits P. Lerche VVDStRL 21 (1964), 66 (74f.); zurückhaltend auch M. Burgess (Fn. 4), 240; anders K. Bretz (Fn. 22), 48 ff. m.w.N.

${ }_{53}$ D. Sauerland Föderalismus zwischen Freiheit und Effizienz, 1997, 16f.; Th. Apolte Die ökonomische Konstitution eines föderalen Systems, 1999, 172; G. Schick Doppelter Föderalismus in Europa, 2003, 166.

54 So bereits H.-U. Derlien DV 7 (1974), 1 (22); M. Boerger Die Effizienz öffentlicher Verwaltung in der Bundesrepublik Deutschland, 1978, 142; s.a. D. Stahl Aufgabenverteilung zwischen Bund und Ländern, 2000, 142: „Die ökonomische Theorie bietet kein Raster, welches eine rein wissenschaftliche Beantwortung der Frage nach der Zuordnung einer bestimmten Aufgabe ermöglicht.“; für das Subsidiaritätsprinzip auch S. Bartolini (Fn. 21), 152. 
Mischverwaltung qualifiziert.55 Das Dogma, dass diese grundsätzlich unzulässig ist, ist allerdings schon im Grundgesetz nicht eindeutig verankert und hat in der Rechtsprechung des Bundesverfassungsgerichtes keine klaren Konturen gefunden. ${ }^{56}$ Richtigerweise ist allein der Verfassungsvorbehalt des Art. 83 GG relevant, wonach Abweichungen vom Grundsatz der Länderexekutive von einer Ermächtigung im Grundgesetz selbst abhängig sind. ${ }^{57}$ Ebenenübergreifende Organisationen bleiben in Bundesstaaten deshalb auf Ausnahmefälle beschränkt. Daraus folgt aber kein konsequentes Gebot der Ebenentrennung, wie die verschiedenen Beispiele für die Mitwirkung von Ländervertretern in Bundesbehörden und von kommunalen Vertretern in Landesgremien zeigen, für die keine explizite verfassungsrechtliche Grundlage besteht.

Im Verhältnis zwischen Gemeinschaft und Mitgliedstaaten bilden die verschiedenen Mischformen dagegen ein wesentliches Element, das als dezentralisierte Integration bezeichnet worden ist. ${ }^{58}$ Das Primärrecht begrenzt solche Kooperationsverhältnisse nicht, da der Rat in die Durchführung des Gemeinschaftsrechts einbezogen werden kann (Art. 202 tir. 3 EGV), um die politische Kontrolle durch die Mitgliedstaaten zu sichern. ${ }^{59}$ Formen der unvollständigen Zentralisierung durch eine Beteiligung der dezentralen Verwaltungen in Ausschüssen können aber auch Effizienzgewinne bieten, indem sie die Möglichkeit eines ständigen Erfahrungsaustauschs zwischen der Kommission und den nationalen Behörden schaffen und damit einen Lernmechanismus institutionalisieren. ${ }^{60}$ Insbesondere in Bereichen der Risikoverwaltung wie etwa

55 W. Kahl DV 29 (1996), 341 (373); A. Hatje Die gemeinschaftsrechtliche Steuerung der Wirtschaftsverwaltung, 1998, 47; H. P. Nehl Europäisches Verwaltungsverfahren und Gemeinschaftsverfassung, 2002, 33; B. Schöndorf-Haubold (Fn. 35), 466f.; G. Winter EuR 2005, 255 (258).

56 Vgl. großzügig BVerfGE 63, 1 (38); restriktiv BVerfGE 108, 169 (182); zur Herleitung und Kritik T. Hebeler in: Bauschke u.a. (Hrsg.) Pluralität des Rechts - Regulierung im Spannungsfeld der Rechtsebenen, 2002, 37.

57 S. Oeter Integration und Subsidiarität im deutschen Bundesstaat, 1998, 453; Th. Groß in: Friauf/Höfling (Hrsg.) BerlK-GG, Art. 83 Rn. 31; in Österreich wird die Schaffung gemeinschaftlicher Einrichtungen mit Entscheidungskompetenzen ohne verfassungsrechtliche Grundlage ebenfalls für unzulässig gehalten, vgl. B.-Ch. Funk VVDStRL 46 (1988), 57 (85).

58 E. Chiti ERPL/REDP 2002, 1267.

59 Zum Ermessensspielraum bei der Delegation auf die Kommission vgl. J. Ch. Wichard in: Calliess/Ruffert (Hrsg.) EUV/EGV, 2. Aufl. 2002, Art. 202 Rn. 5; J. P. Jacqué in: von der Groeben/Schwarze (Hrsg.) EU-/EG-Vertrag, 6. Aufl. 2004, Art. 202 Rn. 12.

${ }^{60}$ K.-H. Ladeur NuR 1997, 8 (14); Ch. Joerges EuR 2002, 17 (34); G. Winter EuR 2005, 255 (267); A. Benz in: Blanke/v. Bandemer/Nullmeier/Wewer (Hrsg.) Handbuch zur Verwaltungsreform, 3. Aufl. 2005, 18 (21). 
bei der Arzneimittelzulassung und der Regulierungsverwaltung kann dies eine sinnvolle Funktion netzwerkartiger Strukturen sein, die Vorteile gegenüber einem rein nationalen Vollzug bieten. Zudem können Formen kooperativer Verwaltung zur Schonung der Autonomie der dezentralen Ebene das mildere Mittel gegenüber einer vollständigen Zentralisierung bilden und damit dem Subsidiaritätsprinzip dienen. ${ }^{61}$

\section{Die Ausgestaltung der Ebenenkoordination}

\section{Die Typen der vertikalen Koordination}

Auch wenn die dezentrale Organisation der Gesetzesanwendung keinen generellen Vorrang genießt, spielt sie in der Praxis eine wesentliche Rolle. Dadurch entsteht ein Kontrollbedürfnis, da es dem zentralen Normgeber nicht gleichgültig sein kann, ob die von ihm erlassenen Vorschriften beachtet und wie sie angewendet werden. Außerdem hat er ein Interesse an Informationen aus dem Vollzug, um die Tauglichkeit der Regelungen überprüfen zu können. Deshalb ist in jeder vollzugsföderalistischen Ordnung die Eigenverantwortlichkeit der unteren Ebenen mehr oder weniger relativiert. Allerdings ist es meistens nicht das gesetzgebende Organ, sondern die seiner Ebene zugeordnete Exekutive, der die entsprechenden Kontrollbefugnisse zustehen. Sie werden in der deutschen Dogmatik v.a. mit dem Begriff der „Aufsicht" 62 erfasst. Will man allerdings allgemeiner die Koordination mehrerer Verwaltungsebenen erfassen, empfiehlt es sich, sechs Typen von Instrumenten zu unterscheiden: Informationsaustausch, Rechtskontrolle, abstrakt-generelle Normkonkretisierung, Einzelweisung, Übernahme der Zuständigkeit und gemeinsame Entscheidungsverfahren.

Voraussetzung jeder Art von präventiver oder repressiver Einflussnahme ist der Informationsaustausch, um der übergeordneten Ebene Kenntnisse über die Vollzugspraxis zu verschaffen. ${ }^{63}$ Ein allgemeines Unterrichtungsrecht der Aufsichtsbehörde ist im Kommunalrecht vorgesehen, ${ }^{64}$

61 G. Sydow (Fn. 10), 47; ähnlich L. Hooghe/G. Marks (Fn. 8), 114; G. Britz EuR 2006, 46 (50); vgl. bereits J. A. Frowein VVDStRL 31 (1973), 13 (43), für die Gemeinschaftsaufgaben.

62 Grundlegend W. Kahl Die Staatsaufsicht, 2000, 347 ff.; s.a. S. U. Pieper Aufsicht, 2006, 9 ff.; F. Schoch Jura 2006, 188, 358.

${ }^{63}$ A. Gil Ibáñez (Fn. 13), 63.

${ }^{64}$ Z.B. $\S 137$ GO Hessen, Art. 119a Abs. 4 B-VG, zu den kantonalen Regelungen in der Schweiz vgl. R. Jagmetti ZSR 1972 II, 321 (354ff.). 
daneben gibt es besondere Anzeigepflichten. ${ }^{65}$ Im Bund-Länder-Verhältnis besteht eine Aktenvorlagepflicht nur im Bereich der Auftragsverwaltung (Art. 85 Abs. 4 S. 2 GG), während sonst lediglich Beauftragte entsendet werden können (Art. 84 Abs. 3 S. 2 GG). ${ }^{66}$ In Österreich sind entsprechende Informationsinstrumente materienspezifisch geregelt (z.B. Art. 11 Abs. 9, 14 Abs. 8 B-VG), sie ergeben sich aber auch aus dem allgemeinen Aufsichtsrecht des Bundes. ${ }^{67}$ In der Schweizer Verfassung ist pauschal vorgesehen, dass der Bund die Einhaltung des Bundesrechts durch die Kantone überwacht (Art. 49 Abs. 2, Art. 186 Abs. 4 BV), woraus sich auch Auskunftspflichten ergeben. ${ }^{68}$ Besonders ausführlich sind die Regelungen im Gemeinschaftsrecht. Neben dem allgemeinen Auskunftsrecht der Kommission (Art. 284 EGV) enthalten die Sekundärrechtsakte häufig spezielle Berichtspflichten der Mitgliedstaaten ${ }^{69}$ sowie Inspektionsrechte der Kommission ${ }^{70}$ oder auch von Agenturen. ${ }^{71}$ Die Schaffung eines Informationsverbundes zwischen den Verwaltungen, in vielen Bereichen gestützt durch spezielle elektronische Datennetze, ${ }^{72}$ ist ein zentrales Element des europäischen Regulierungskonzeptes. ${ }^{73}$

Wird gegenüber einem politisch selbständigen Verwaltungsträger „Aufsicht" ausgeübt, so ist sie überwiegend auf eine Rechtskontrolle beschränkt. Die für die Rechtsetzung zuständige übergeordnete Ebene darf sich grundsätzlich nicht in Anwendungsspielräume einmischen, sondern nur überprüfen, ob die normativen Vorgaben eingehalten werden. Für die Kommunen ergibt sich dies aus der verfassungsrechtlichen Garantie der Selbstverwaltung (Art. 28 Abs. 2 S. 1 GG, Art. 119a Abs. 1 B-VG, Art. 50 BV). ${ }^{74}$ Im Bund-Länder-Verhältnis bestehen entsprechende Beschränkungen in Deutschland beim eigenverantwortlichen Ländervollzug (Art. 84 Abs. 3 S. 1 GG) ebenso wie in der Schweiz

65 Z.B. $\$ 97$ Abs. 4 GO Hessen für den Haushaltsplan der Gemeinde.

66 Dies umfasst eine Auskunftspflicht, vgl. G. Hermes (Fn. 51), Art. 84 Rn. 81.

67 Vgl. K. Weber (Fn. 26), $240 \mathrm{ff}$.

68 P. Forster in: Fleiner/Forster/Misic/Thalmann (Fn. 48), 131 (135); A. Ruch in: Ehrenzeller u.a. (Hrsg.) Die schweizerische Bundesverfassung, 2002, Art. 186 Rn. 18.

${ }^{69}$ Dazu A. Hatje (Fn. 55), 144 f.; J. Sommer Verwaltungskooperation am Beispiel administrativer Informationsverfahren im Europäischen Umweltrecht, 2003, $134 \mathrm{ff}$.

${ }^{70}$ Dazu ausführlich A. David (Fn. 23), passim.

71 Vgl. Th. Groß EuR 2005, 54 (63).

72 A. v. Bogdandy in: Hoffmann-Riem/Schmidt-Aßmann (Hrsg.) Verwaltungsrecht in der Informationsgesellschaft, 2000, 133 (175f.); ausführlich M. Eifert Electronic Government, 2006, $425 \mathrm{ff}$.

${ }^{73} \mathrm{Vgl}$. Ch. Koch Arbeitsebenen der Europäischen Union, 2003, $312 \mathrm{ff}$.

${ }^{74} \mathrm{Vgl}$. zur Abhängigkeit der Garantie der Gemeindeautonomie vom kantonalen Recht R. Kägi-Diener in: Ehrenzeller u.a. (Fn. 68), Art. 50 Rn. 6. 
(Art. 186 Abs. 4 BV), ${ }^{75}$ nicht aber im stärker unitarisch geprägten Österreich. In der Europäischen Gemeinschaft beschränkt sich das von der Kommission einzuleitende Vertragsverletzungsverfahren gegenüber den Mitgliedstaaten (Art. 226 Abs. 1 EGV) ebenfalls auf die Überprüfung von Rechtsverstößen. ${ }^{76}$

Ein weitergehendes Instrument hierarchischer Einwirkung ist der Erlass abstrakt-genereller Anleitungen zur Gesetzesanwendung. Da sie im Mehrebenensystem nicht auf eine allgemeine Überordnung gestützt werden können, bedürfen sie einer ausdrücklichen Rechtsgrundlage. Gegenüber der kommunalen Selbstverwaltung ist eine Bindung an Verwaltungsvorschriften folglich von einer gesetzlichen Ermächtigung abhängig. ${ }^{77}$ Im föderalen Verhältnis bietet nur das Grundgesetz (Art. 84 Abs. 2, 85 Abs. 2 GG) eine klare verfassungsrechtliche Grundlage. In Österreich werden sie als generelle Weisungen klassifiziert. ${ }^{78}$ In der Schweiz erfüllen die Kreisschreiben des Bundesrates die gleiche Funktion. Für sie gibt es allerdings keine explizite verfassungsrechtliche Grundlage, sie wird entweder in der Kompetenz für die Bundesaufsicht ${ }^{79}$ oder für die Bundesgesetzgebung ${ }^{80}$ gefunden. Im Primärrecht der Gemeinschaft fehlt jeder Hinweis auf ein vergleichbares Instrument der administrativen Normkonkretisierung. Dennoch hat die Kommission eine Vielzahl von Mitteilungen und Leitlinien zur Lenkung des mitgliedstaatlichen Verwaltungsvollzugs erlassen, denen zum Teil über das Institut der Selbstbindung, zum Teil kraft ausdrücklicher Anordnung in Sekundärrechtsakten eine zumindest inneradministrative Verbindlichkeit zukommt, die es rechtfertigt, von einer tertiären Rechtsschicht der Vollzugsregelungen zu sprechen. ${ }^{81}$ Offensichtlich gibt es in allen Relationen

${ }^{75} \mathrm{Ob}$ durch Gesetz auch eine Ermessenskontrolle gegenüber den Kantonen eingeführt werden kann, ist strittig, vgl. U. Häfelin/W. Haller (Fn. 36), Rn. 1208.

76 W. Kahl (Fn. 62), 400; W. Cremer in: Calliess/Ruffert (Fn. 59), Art. 226 EGV Rn. 2.

77 BVerfGE 76, 107 (117f.); für das Haushaltsrecht BVerwG, NVwZ-RR 1989, 377; zu pauschal F. Ossenbühl Verwaltungsvorschriften und Grundgesetz, 1968, 386ff.; für Art. 84 Abs. 2 GG zutreffend Th. Sauerland Die Verwaltungsvorschrift im System der Rechtsquellen, 2005, $184 \mathrm{ff}$.

${ }^{78}$ R. Mayer/H. Mayer Grundriß des österreichischen Bundesverfassungsrechts, 8. Aufl. 1996, Rn. 1104.

79 U. Häfelin/W. Haller (Fn. 36), Rn. 1212; Th. Jaag in: Thürer/Aubert/Müller (Hrsg.) Verfassungsrecht der Schweiz - Droit constitutionnel suisse, 2001, § 30 Rn. 40.

80 Vgl. G. Biaggini Theorie und Praxis des Verwaltungsrechts im Bundesstaat, 1996, $80 \mathrm{ff}$.

81 Th. Groß DÖV 2004, 20; aufgenommen bei G. Sydow (Fn. 10), 52 ff.; H. SchulzeFielitz (Fn. 12), 121; zur Parallele zu den Verwaltungsvorschriften vgl. S. Schlacke Risikoentscheidungen im europäischen Lebensmittelrecht, 1998, 259; A. Hatje (Fn. 55), 
eine funktionale Notwendigkeit, den Gesetzesvollzug untergeordneter Ebenen mit einem flexiblen Instrument der abstrakt-generellen Normkonkretisierung zu regeln.

Eine davon $\mathrm{zu}$ unterscheidende und nicht notwendig mit ihr verknüpfte Form der hierarchischen Steuerung ist das Instrument der Einzelweisung. Sie ist gegenüber kommunalen Körperschaften nur nach Maßgabe einer gesetzlichen Ermächtigung zulässig (z.B. Art. 137 Abs. 4 LV Hessen, Art. 119 Abs. 1 B-VG). Im Bund-Länder-Verhältnis sind die Regelungen unterschiedlich. In Deutschland sind Einzelweisungen nur für besondere Fälle oder im Rahmen der Bundesauftragsverwaltung zulässig (Art. 84 Abs. 5, 85 Abs. 3 GG). In Österreich ist dagegen die Weisungsunterworfenheit der Organe der Länder die Regel, hier bedürfen Ausnahmen einer verfassungsrechtlichen Grundlage (Art. 20 Abs. 1, 103 Abs. 1 B-VG). ${ }^{82}$ In der Schweiz besteht keine allgemeine verfassungsrechtliche Regelung, doch gilt es als zulässig, dass Bundesgesetze eine Weisungsbefugnis von Bundesbehörden gegenüber den Kantonen vorsehen. ${ }^{83}$ Ebenso fehlt im Gemeinschaftsrecht eine abstrakte Regelung, doch erfüllt insbesondere die Möglichkeit einer staatengerichteten Entscheidung der Kommission die gleiche Funktion. ${ }^{84}$ Auch Empfehlungen und Mitteilungen können weisungsähnlich wirken. ${ }^{85}$

Ein noch weitergehender Eingriff ist die Übernahme der Entscheidungszuständigkeit durch die zentrale Verwaltung. Im Kommunalrecht ist sie unter dem Begriff des Selbsteintrittsrechtes bekannt, das eine gesetzliche Grundlage erfordert. ${ }^{86}$ Im Verhältnis zwischen Bundes- und Landesverwaltung ist sie dagegen in allen drei Ländern nicht vorgesehen. Im Gemeinschaftsrecht kann jedoch die Kommission z. B. Kartellverfahren an sich ziehen (Art. 11 Abs. 6 Kartell-VO). ${ }^{87}$

$158 \mathrm{ff}$; $M$. Vogt Die Entscheidung als Handlungsform des Europäischen Gemeinschaftsrechts, 2005, 227.

${ }^{82}$ Dazu W. Barfuß Die Weisung, 1967, 22.

83 Vgl. G. Biaggini (Fn. 80), 116.

${ }^{84}$ W. Pühs Der Vollzug von Gemeinschaftsrecht, 1997, $295 \mathrm{ff} . ;$ F. Wettner (Fn. 16), $177 \mathrm{ff}$.

85 W. Pühs (Fn. 84), 298; B. Schöndorf-Haubold (Fn. 35), 321; s. a. A. David (Fn. 23), 252.

86 Vgl. VGH Mannheim, NVwZ-RR 1992, 602 (603); OVG Weimar, LKV 1993, 428 (429); U. Guttenberg Weisungsbefugnisse und Selbsteintritt: eine verwaltungsorganisationsrechtliche Untersuchung, 1992, 244 ff., allerdings mit problematischen Einschränkungen.

87 Dazu G. Sydow (Fn. 10), 159; A. Fuchs EuR Beiheft 2/2005, 77 (82); H. Ch. Röhl DVB1. 2006, 1070 (1076); zur ähnlichen „Hochzonung“ nach der Novel-Food-VO vgl. D. Groß Die Produktzulassung von Novel Food, 2001, $178 \mathrm{ff}$. 
Als sechste selbständige Koordinationsform sind gestufte Verfahren einer gemeinsamen Entscheidung aufzufassen. ${ }^{88}$ Durch Genehmigungsvorbehalte und Einvernehmenserfordernisse wird die Wirksamkeit einer Entscheidung der untergeordneten Ebene von einem Mitwirkungsakt der übergeordneten Ebene abhängig gemacht. Im Gegensatz zur einseitigen Weisung ist hier aber ein selbstständiger Kooperationsbeitrag der untergeordneten Ebene erforderlich. $\mathrm{Ob}$ es sich nur um eine präventive Rechtskontrolle handelt oder auch Zweckmäßigkeitserwägungen zulässig sind, bestimmt sich nach dem jeweiligen Regelungsprogramm. Im Kommunalrecht sind Genehmigungsvorbehalte nach Maßgabe des Gesetzesvorbehaltes zulässig. ${ }^{89} \mathrm{Im}$ Bund-Länder-Verhältnis sind sie dagegen nur insoweit möglich, als auch Einzelweisungen zulässig wären, da es sich um graduell geringere Eingriffe handelt. ${ }^{90}$ Auch im Gemeinschaftsrecht sind vergleichbare Zustimmungserfordernisse der Kommission zu finden. Auf primärrechtlicher Basis gilt dies etwa bei der Billigung nationaler Schutzklauseln im Binnenmarkt (Art. 95 Abs. 6 EGV). Im Sekundärrecht finden sich viele weitere Beispiele. ${ }^{91}$

\section{Die Durchsetzungsmechanismen}

Ein eigenes Regelungsproblem sind die Mechanismen zur Durchsetzung der Koordinationsmaßnahmen im Konfliktfall. Administrative Eingriffsbefugnisse der höheren Ebene gibt es v.a. im Kommunalrecht, wo kassatorische Entscheidungen und die Ersatzvornahme als Instrumente des unmittelbaren Durchgriffs durch die Aufsichtsbehörde selbstverständlich sind. ${ }^{92}$ Im Bund-Länder-Verhältnis sind sie erstaunlicherweise in der Schweiz anerkannt, wo trotz einer sehr vagen verfassungstextlichen Grundlage die Aufhebung von kantonalen Hoheitsakten

88 Vgl. Th. Groß DVB1. 2002, 793 (798f.).

${ }^{89}$ Dazu näher E. Schmidt-Aßmann/H. Ch. Röhl in: Schmidt-Aßmann (Hrsg.) Besonderes Verwaltungsrecht, 13. Aufl. 2005, 1. Abschnitt Rn. 48f.; für Österreich vgl. Art. 119a Abs. 8 B-VG.

90 Vgl. zu Art. 84 GG BVerwGE 42, 279 (284); zu Art. 85 GG R. Steinberg AöR 110 (1985), 419 (428f.); zur Schweiz vgl. Art. 186 Abs. 2 BV. In Österreich stellt sich die Frage aufgrund der generellen Weisungshierarchie nicht.

$91 \mathrm{Vgl}$. zu gestuften Verfahren im Zollrecht $A$. Hatje (Fn. 55), $161 \mathrm{f}$.; im Umweltrecht J. Sommer (Fn. 69), 277 ff.; im TK-Recht K. Herzmann ZNER 2005, 216 (219); im Energierecht G. Britz EuR 2006, 46 (65); allgemein J.-P. Schneider EuR Beiheft 2/2005, 141; H. Ch. Röhl DVB1. 2006, 1070 (1074ff.).

92 Vgl. E. Schmidt-Aßmann/H. Ch. Röhl (Fn. 89), Rn. 42, auch zu weiteren Mitteln wie der Auflösung des Gemeinderates; zu Österreich vgl. Art. 119a Abs. 6 S. 2, Abs. 7 S. 2 B-VG; zur Schweiz vgl. R. Jagmetti ZSR 1972 II, 321 (354ff.). 
und die Ersatzvornahme als Mittel der Bundesaufsicht als zulässig angesehen werden, auch wenn in der Praxis kaum von ihnen Gebrauch gemacht wird. ${ }^{93}$ Dagegen fehlen entsprechende Instrumente in den beiden anderen Bundesstaaten, so dass Auseinandersetzungen über Rechte und Pflichten der Gliedstaaten dort vor Gericht ausgetragen werden müssen. Gleiches gilt für die Kommission, die Implementationsdefizite der Mitgliedstaaten grundsätzlich nur über das Vertragsverletzungsverfahren vor dem Gerichtshof rügen kann. In einigen Bereichen hat das Gemeinschaftsrecht allerdings ein gestuftes Kontrollsystem geschaffen, in dem die Kommission eine Art Strukturaufsicht als „Kontrolle der Kontrolle“ ausüben kann. ${ }^{94}$ Zudem hat sie im Bereich der Agrarsubventionen mit dem Rechnungsabschlussverfahren finanzielle Sanktionsmittel gegenüber regelverletzenden Mitgliedstaaten, die eine eigenständige effektive Durchsetzung ermöglichen. ${ }^{95}$ Dies ist allerdings ein Sonderfall, im Allgemeinen sind administrative Durchgriffsmöglichkeiten auf Einzelentscheidungen in einem föderalen Verhältnis ausgeschlossen.

In der hierarchischen Verwaltungsorganisation wird die Fachaufsicht durch Instrumente der Dienstaufsicht flankiert, die Sanktionen gegenüber dem einzelnen Amtsinhaber ermöglichen. In Mehrebenensystemen ist dagegen eine solche übergreifende Personalgewalt nur ganz ausnahmsweise vorgesehen, etwa bei der Dienstaufsicht gegenüber kommunalen Ordnungsbehörden, ${ }^{96}$ während sie im Bereich der Rechtsoder Fachaufsicht gegenüber Kommunen ansonsten ausgeschlossen ist. Gleiches gilt generell im Bund-Länder-Verhältnis. ${ }^{97}$ In Österreich ist aber eine Anklage von Mitgliedern der Landesregierung vor dem Verfassungsgerichtshof möglich (Art. $142 \mathrm{~B}-\mathrm{VG}$ ). ${ }^{98}$ Erst recht ist ein Zugriff der Gemeinschaftsorgane auf dienstrechtliche Befugnisse der Mitgliedstaaten ausgeschlossen. ${ }^{99}$

93 U. Häfelin/W. Haller (Fn. 38), Rn. 1223, 1234; Th. Jaag (Fn. 79), § 30 Rn. 42, 44; A. Ruch (Fn. 68), Art. 186 Rn. 18.

${ }^{94}$ A. David (Fn. 23), 119f.; E. Schmidt-Aßmann Das allgemeine Verwaltungsrecht als Ordnungsidee, 2. Aufl. 2004, 232; R. Streinz EuR Beiheft 2/2005, 7 (15).

$95 \mathrm{Vgl}$. E. Pache Der Schutz der finanziellen Interessen der Europäischen Gemeinschaften, 1994, 278 ff.; A. Hatje (Fn. 55), 163 ff.; W. Schenk (Fn. 12), $242 \mathrm{ff}$.

96 Vgl. z.B. § 86 Abs. 2 und 4 SOG Hessen, § 63 Abs. 1 PolG Baden-Württemberg.

${ }^{97}$ Die Entsendung von Beauftragten nach Art. 84 Abs. 3 S. 2, 85 Abs. 4 S. 2 GG führt nicht zu einer Ersetzung des landeseigenen Personals, da sie nur Aufklärungsfunktionen haben, vgl. H. P. Bull in: Denninger/Hoffmann-Riem/Schneider/Stein (Hrsg.) Alternativkommentar zum Grundgesetz, 3. Aufl. 2001, Art. 85 Rn. 27.

${ }_{98}$ Dazu $K$. Weber (Fn. 26), $232 \mathrm{ff}$.

${ }^{99}$ A. Gil Ibáñez (Fn. 13), 288. Davon abzugrenzen sind für das Dienstrecht relevante sekundärrechtliche Bestimmungen, die für bestimmte nationale Verwaltungsstellen wie z.B. die Kontrollstellen im Tabaksektor, die Datenschutzbeauftragten oder die Regulie- 
Nur noch ein Relikt stellen der deutsche Bundeszwang (Art. 37 GG) und die militärische Bundesexekution nach Schweizer Verfassungsrecht ${ }^{100}$ dar. Eine Ermächtigung zu einem Gewalteinsatz gegen föderale Gliedstaaten ist in einer modernen Verfassungsordnung überflüssig, wie nicht nur ihre fehlende Praxisrelevanz, sondern auch die Verfassung Österreichs belegt, die kein vergleichbares Instrument kennt. Die EU hat ebenfalls mehr als fünf Jahrzehnte als bloße Rechtsgemeinschaft ohne Gewaltmittel funktioniert, ${ }^{101}$ ohne dass die Implementationsdefizite unerträglich groß geworden wären. In modernen föderalen Systemen reicht es aus, dass Meinungsverschiedenheiten durch die jeweilige Verfassungsgerichtsbarkeit geklärt werden können. ${ }^{102}$

Neben diesen exekutiven Mechanismen gibt es weitere Instrumente, mit denen die Durchsetzung von Rechtsnormen durch externe Kontrollen gefördert werden kann. Ein wesentliches Element ist die „Mobilisierung der Bürger“, eine insbesondere das EG-Umweltrecht prägende Tendenz, die Bürger der Mitgliedstaaten, einschließlich privater Verbände, mit Informations- und Klagerechten auszustatten, um ihnen eine Kontrolle der staatlichen Behörden und damit auch der Einhaltung der umweltrechtlichen Vorschriften zu ermöglichen. ${ }^{103}$ Daneben können vorgelagerte private Fremdkontrollen eingeführt werden, wie z.B. im Versicherungsaufsichtsrecht. ${ }^{104}$

\section{Die Schaffung von Legitimation als Gestaltungsaufgabe}

\section{Die Verantwortungsverteilung im Mehrebenensystem}

Die polyzentrische Mehrebenenverwaltung, deren Koordinationsinstrumente soeben skizziert wurden, führt notwendig zu einer Verteilung der Verantwortung, die mit dem traditionellen monistischen Modell der

\footnotetext{
rungsbehörden eine unabhängige Stellung vorschreiben. Diese Regelungen betreffen allerdings Dienststellen, nicht einzelne Personen.

${ }^{100}$ Zur nicht ausdrücklich geregelten Verankerung in der Bundesverfassung vgl. U. Häfelin/W. Haller (Fn. 38), Rn. 1240f.; Th. Jaag (Fn. 79), § 30 Rn. 45.

101 W. Pühs (Fn. 84), 130; A. v. Bogdandy (Fn. 5), 37; s.a. A. Peters (Fn. 24), 155 ff.

102 So schon H. Kelsen ZÖR Bd. VI (1927), 329 (350); zum Einsatz von Armee oder Polizei, wenn einem Urteil im Einzelfall nicht Folge geleistet wird, vgl. Art. 146 Abs. 2 B-VG.

${ }^{103}$ Begriffsprägend J. Masing Die Mobilisierung des Bürgers für die Durchsetzung des Rechts, 1997; der Grundgedanke findet sich schon bei C.-D. Ehlermann, LA Pescatore, 1987, 205 (217 ff.).

104 Dazu M. Eifert GVwR I, § 19 Rn. 86f.
} 
Verwaltungslegitimation ${ }^{105}$ in Konflikt gerät. Nur im Einheitsstaat kann man die sachliche und die personelle Legitimation auf eine gemeinsame Quelle zurückführen: Das vom Parlament beschlossene Gesetz wird von einer Verwaltung ausgeführt, deren Personal von der Regierung ausgewählt, für konkrete Aufgaben eingesetzt und durch Weisungen gelenkt wird. Die Regierung wird ihrerseits von diesem Parlament bestimmt und ist ihm gegenüber verantwortlich. In dieser idealtypischen Konstellation kann man die Ausführung des Gesetzes als eine Form des Willenstransfers verstehen, bei dem der Wille des Gesetzgebers, der Ausdruck des Volkswillens ist, durch die politisch gleich ausgerichtete Exekutivspitze konkretisiert wird, während dem nachgeordneten Behördenapparat keine eigenständige Rolle im Implementationsprozess zugestanden wird. ${ }^{106}$

Dieses Modell ist, ganz abgesehen von der Überschätzung der ministerialen Steuerungskapazitäten, ${ }^{107}$ für eine Mehrebenenverwaltung mit verteilten Kompetenzen nicht tauglich. ${ }^{108}$ Schon im deutschen Bundesstaat mit seiner starken kommunalen Selbstverwaltung überkreuzen sich die Legitimationsmodi regelmäßig. Die meisten verwaltungsrechtlichen Gesetze werden auf Bundesebene erlassen. Ihre Ausführung erfolgt jedoch in der Regel durch die Länder, welche die erstinstanzliche Zuständigkeit häufig der kommunalen Ebene als untere Verwaltungsbehörde überlassen. Die personelle Legitimation der handelnden Amtsträger erfolgt dementsprechend durch die Kommunalwahlen. Da sie allerdings regelmäßig der Weisungsbefugnis der Landesverwaltung unterstehen, besteht bei der konkreten sachlichen Legitimation eine Verantwortlichkeit gegenüber dem Landesparlament, das politisch wiederum anders ausgerichtet sein kann als das Bundesparlament, das mit dem Gesetz die generelle sachliche Legitimation vorgibt. An wessen „Willen“ soll sich die Beamtin im Landratsamt nun orientieren? Folgt sie der politischen Mehrheit im Kreis, von der sie ihr Amt erhalten hat, folgt sie der poli-

105 Vgl. die Rekonstruktion bei H.-H. Trute GVwR I, § 6 Rn. $4 \mathrm{ff}$.

106 Prägnant C. F. v. Gerber Grundzüge des deutschen Staatsrechts, 3. Aufl. 1880, 77 : „Nicht zu den Organen des Staats gehören die Staatsdiener; ihr Recht ist kein ursprüngliches Recht zur Kundgebung des Staatswillens, sondern ein abgeleitetes, sie sind nur die rechtlichen Gehülfen des Monarchen. ... Der Monarch ist das oberste Willensorgan des Staates."; eine moderne Variante bei H. Dreier Hierarchische Verwaltung im demokratischen Staat, 1991, 129 ff.; zur Kritik vgl. Th. Groß (Fn. 12), $168 \mathrm{ff}$.

107 V. Mehde Neues Steuerungsmodell und Demokratieprinzip, 2000, 446ff.

${ }^{108}$ H. P. Nehl (Fn. 55), $120 \mathrm{ff}$;; G. Sydow (Fn. 10), 239f.; skeptisch auch H. Ch. Röhl DVB1. 2006, 1070 (1079); H. Schulze-Fielitz (Fn. 12), 128; s.a. für Österreich K. Weber (Fn. 26), $357 \mathrm{ff}$. 
tischen Linie der Landesregierung, auch sofern sie keine Weisung erhält, oder folgt sie der politischen Ausrichtung des Bundestages, der das Gesetz erlassen hat?

Ein Modell, das von einer einheitlichen Legitimationsquelle im Volkswillen ausgeht, ist unterkomplex. ${ }^{109}$ Weitere Schwierigkeiten entstehen, wenn die europäische Ebene hinzugenommen wird. Ist eine von Rat und Parlament erlassene Richtlinie Ausdruck des „Willens“ der Völker der in der Gemeinschaft zusammengeschlossenen Staaten (vgl. Art. 189 EGV)? Auch die transnationalen Wirkungen von Verwaltungsentscheidungen, die in einigen Bereichen des Gemeinschaftsrechts angeordnet und oft mit horizontalen Koordinationsverfahren verbunden werden, ${ }^{110}$ sind kaum zu rechtfertigen, wenn man die nationale personelle Legitimation in den Mittelpunkt stellt.111 Man gerät hier in unlösbare Probleme, deren Ursache aber in einer untauglichen Konzeption und nicht in einer Demokratieunfähigkeit mehrfach gestufter Staatlichkeit liegt.

\section{Das institutionelle Legitimationsarrangement}

Ein realitätsgerechtes Modell muss von dem Pluralismus der Legitimationsquellen ausgehen, in dem viele unterschiedliche Akteure an einem arbeitsteiligen Implementationsprozess beteiligt sind. In Mehrebenensystemen gibt es kein Willenszentrum, sondern ein Zusammenwirken verschiedener öffentlicher Stellen, das keinem einheitlichen Konzept folgt. ${ }^{112}$ Ein Verantwortungszusammenhang zwischen den Ebenen ergibt sich zwar durch die genannten Koordinationsinstrumente, die von der übergeordneten Ebene aufgabenspezifisch in variablen Kombinationen eingesetzt werden können, um den Vollzug auf den untergeordneten Ebenen mehr oder weniger detailliert zu kontrollieren und $\mathrm{zu}$

109 G. Lübbe-Wolff VVDStRL 60 (2001), 246 (279ff.); N. Bernard Multilevel Governance in the European Union, 2002, 255; U. Schliesky Souveränität und Legitimität von Herrschaftsgewalt, 2004, $241 \mathrm{ff}$; A. Hanebeck Der demokratische Bundesstaat des Grundgesetzes, 2004, $271 \mathrm{ff}$; nach $A$. Tschentscher Demokratische Legitimation der dritten Gewalt, 2006, 24, ist der Volkswille „fiktiv“ im positiven Sinn einer regulativen Idee.

110 Vgl. V. Neßler, Europäisches Richtlinienrecht wandelt deutsches Verwaltungsrecht, 1994; G. Sydow (Fn. 10), $138 \mathrm{ff}$.

111 Vgl. die Problematisierungen bei H. Ch. Röhl Akkreditierung und Zertifizierung im Produktsicherheitsrecht, 2000, $44 \mathrm{ff}$., der von einer „strukturellen Legitimation“ spricht; G. Sydow (Fn. 10), $242 \mathrm{ff}$.

112 H.-H. Trute GVwR I, § 6 Rn. 32; G. Frankenberg in: ders. Autorität und Integration, 2003, 73 (114), weist zu Recht auf die Notwendigkeit der Anerkennung gestufter kollektiver Identitäten hin. 
vereinheitlichen. ${ }^{113}$ Es gibt aber weder einen generellen Vorrang der hierarchischen Koordination, ${ }^{114}$ da sie eine Mehrheitsherrschaft voraussetzt, die in föderalen Ordnungen nie ungebrochen durchgeführt werden kann, ${ }^{115}$ noch eine uneingeschränkte Autonomie der dezentralen Ebenen.

Damit muss auch die Fixierung der Legitimationstheorie auf die parlamentarische Verantwortlichkeit der Verwaltungsspitze in Frage gestellt werden. In einem überwiegend dezentralen Vollzugskonzept ist das Sanktionspotential des zentralen Parlamentes gegenüber den Verwaltungsinstanzen gering. Dass die Volksvertretungen der Gliedstaaten bzw. der Kommunen dieses Defizit durch ihre eigenen Kontrollmechanismen ausgleichen, ist bei einer politischen Entkoppelung vom Gesetzgebungsprozess eher zweifelhaft, da etwa aufgedeckte Mängel der Norm nicht unmittelbar in ihre Korrektur umgesetzt werden können. ${ }^{116}$ Die Furcht vor parlamentarischer Kritik, möglicherweise in der Form eines Untersuchungsausschusses, ist wohl kaum der zentrale Mechanismus, der das gute Funktionieren moderner Verwaltungen garantiert. Parlamentarische Kontrolle kann deshalb nur ein Baustein in einem komplexeren Legitimationskonzept sein. ${ }^{117}$ Neben input- kommen auch outputorientierte Komponenten in Frage, da die strukturelle Gewährleistung einer möglichst optimalen Erfüllung der im Gesetz definierten Aufgaben auch einen demokratischen Wert hat.118

Insbesondere für die Implementationsphase europäischer Politik sind Transparenz und Partizipation wichtige Legitimationsinstrumente, die in erster Linie den organisierten Interessen, aber auch der breiten Öffentlichkeit die Möglichkeit der Mitwirkung an Entscheidungsprozessen bieten. ${ }^{119}$ In gewissem Umfang kann die Fragmentierung politischer

113 Ähnlich M. Fehling EuR Beiheft 2/2005, 41 (59); dazu, dass auch der Informationsaustausch als Kontrollinstrument legitimierend wirken kann, vgl. A. Hatje in: Magiera/Sommermann (Hrsg.), Verwaltung in der Europäischen Union, 2001, 193 (197ff.).

114 G. Sydow (Fn. 10), 248.

115 Skeptisch für die Europäische Union auch H. Steinberger VVDStRL 50 (1991), 9 (49); für eine zentrale Rolle der parlamentarischen Legitimation z. B. W. Abendroth AuBenpolitik 1952, 623 (632); vermittelnd G. Lübbe-Wolff VVDStRL 60 (2001), 246 (259ff.).

116 Ch. Möllers in: Aulehner u.a. (Hrsg.), Föderalismus - Auflösung oder Zukunft der Staatlichkeit?, 1997, 81 (109f.).

117 E. Schmidt-Aßmann (Fn. 94), 402, spricht von einem „Mosaik“.

118 H.-H. Trute GVwR I, § 6 Rn. 53.

119 W. Hoffmann-Riem in: Schmidt-Aßmann/Hoffmann-Riem (Hrsg.), Strukturen des Europäischen Verwaltungsrechts, 1999, 317 (377); A. v. Bogdandy in: ders. (Fn. 5), 149 (178ff.); enger Ch. Möllers CMLR 2006, 313 (321), der nur eine kompensatorische Bedeutung sieht. 
Kontrolle durch Bürgerbeteiligung ausgeglichen werden. ${ }^{120}$ Von zentraler Bedeutung ist dafür zunächst der Anspruch auf Informationszugang, der inzwischen von den Organen der Gemeinschaft (Art. 255 EGV) ${ }^{121}$ auf die Agenturen ausgedehnt worden ist ${ }^{122}$ und auch in den meisten Mitgliedstaaten besteht. ${ }^{123}$ Er bewirkt eine potentielle Politisierbarkeit öffentlicher Angelegenheiten. ${ }^{124}$ Formen der gesellschaftlichen Mitwirkung finden sich auf europäischer Ebene in erster Linie durch die Einbeziehung von Vertretern organisierter Interessen wie den Unternehmensverbänden, den Gewerkschaften oder den Umweltverbänden in beratenden Ausschüssen, ${ }^{125}$ in einigen Agenturen darüber hinaus durch die Entsendung von Mitgliedern in ihr Leitungsorgan. ${ }^{126}$ In der nationalen Verwaltung bestehen vielfältige Partizipationsstrukturen, neben der Kommunalverwaltung ${ }^{127}$ z.B. in der funktionalen Selbstverwaltung, ${ }^{128}$ aber auch in anderen Gremien. ${ }^{129}$

Eine ganz andere technokratische Konzeption versteht die Kommission und die europäischen Ausschüsse als eine Form entpolitisierter Fachverwaltung, in der Experten auf der Grundlage eines ständigen Erfahrungsaustausches nach den besten Lösungen für Sachprobleme suchen. ${ }^{130}$ Ähnlich wird das Erfordernis der Unabhängigkeit nationaler

120 N. Bernard (Fn. 109), 255f.; ähnlich bereits H. Kelsen Allgemeine Staatslehre, 1925, 367, wonach demokratische Formen der Vollziehung in Bereichen weitgehender Ermessensfreiheit neben der autokratischen Vollziehung der Gesetze zu einer Stärkung der Demokratie führen können.

${ }^{121}$ Den Bezug zum „demokratischen Charakter der Organe“ stellt die Erklärung Nr. 17 zum EUV her, auf die auch EuGH, Slg. 1996, I-2169, Rn. 35, und Slg. 2001, I-9565, Rn. 24, verweisen.

122 Th. Groß EuR 2005, 54 (65).

123 Vgl. H.-J. Garstka in: Kloepfer (Hrsg.) Die transparente Verwaltung - Zugangsfreiheit zu öffentlichen Informationen, 2003, 67.

124 M. Höreth Die Europäische Union im Legitimationstrilemma, 1999, $274 \mathrm{ff}$; G. Lübbe-Wolff VVDStRL 60 (2001), 246 (277); F. Schoch DV 35 (2002), 149 (156); E. Schmidt-Aßmann (Fn. 94), 107 ff.; zu den Funktionen auch M. Rossi Informationszugänglichkeit und Verfassungsrecht, 2004, 67ff.; bereits J. H. Kaiser VVDStRL 23 (1966), 1 (25), betonte die Bedeutung der Kommunikation mit der interessierten Öffentlichkeit.

125 A. Peters (Fn. 24), 689; für eine stärkere Einbeziehung intern demokratischer Nichtregierungsorganisationen M. Zürn PVS 1996, 27 (51), auf das Gleichheitsproblem weist auch H. Huget in: Bauer/Voelzkow (Hrsg.), Die Europäische Union - Marionette oder Regisseur?, 2004, 66 (85), hin.

126 Th. Groß (Fn. 12), 343.

127 BVerfGE 79, 127 (149), bezeichnet sie als „Keimzelle der Demokratie“.

128 W. Kluth Funktionale Selbstverwaltung, 1997, $236 \mathrm{ff}$.

129 Vgl. Th. Groß (Fn. 12), $71 \mathrm{ff}$.

130 G. Majone Regulating Europe, 1996, $61 \mathrm{ff}$.; A. Benz PVS 1998, 558 (568); K. Lenaerts/A. Verhoeven in: Joerges/Dehousse (Fn. 13), 35 (51); bereits D. Vignes (Fn. 20), 
Regulierungsbehörden mit der Abschottung gegenüber sachwidrigen Interessen begründet, die auch mittelbar über die politische Leitungsebene in die Einzelentscheidungen hineingetragen werden könnten. ${ }^{131}$ Dieses Modell eignet sich für Aufgabengebiete, in denen nach weitgehend objektivierbaren Effizienzkriterien entschieden werden kann. ${ }^{132}$ Im Bereich der Risikobewältigung ist allerdings deutlich geworden, dass allein das Vertrauen in die Qualität des neutralen Sachverstandes angesichts der Wertungsspielräume nicht ausreichend ist. Folglich können dort Expertenausschüsse bzw. Agenturen regelmäßig keine abschließenden Entscheidungen treffen, sondern diese fallen in die Zuständigkeit der Kommission, die politisch stärker legitimiert ist. ${ }^{133}$

Ein weiterer Steuerungsmechanismus ist der Wettbewerb, der im Bereich der öffentlichen Verwaltung allerdings nur künstlich geschaffen werden kann. Basierend auf dem Konzept des New Public Management, wie es nicht nur im Bereich der Hochschulen eingeführt worden ist, ${ }^{134}$ ist das Ziel, eine Effizienzsteigerung bei der Aufgabenerfüllung dadurch zu erreichen, dass Anreize, insbesondere finanzieller Natur, bei gleichzeitigem Abbau hierarchischer Vorgaben die Leistungen verbessern und Innovation stimulieren. ${ }^{135}$ Auf europäischer Ebene will die „Offene Methode der Koordinierung“ in diesem Sinn einen Wettbewerb der Mitgliedstaaten zur Erreichung vorgegebener Ziele ohne Verwendung verbindlicher Rechtsinstrumente aktivieren. ${ }^{136}$ Die Zielvorgaben und An-

13, spricht von „technocratie“, J. H. Kaiser VVDStRL 23 (1966), 1 (24), von einer „Art intellektueller Aristokratie“.

${ }^{131}$ G. Majone (Fn. 130), 284ff.; K. Oertel Die Unabhängigkeit der Regulierungsbehörde nach $\S 66$ ff. TKG, 2000, 104 ff.; H.-H. Trute GVwR I, § 6 Rn. 68; H. Ch. Röhl JZ 2006, 831 (835); zurückhaltender M. Döhler DV 34 (2001), 59; zu den nur eingeschränkt als Vorbild tauglichen US-amerikanischen agencies z. B. J. Masing AöR 128 (2003), 558 (584ff.).

132 M. Höreth (Fn. 124), 285 ff.

133 O. Blattner Europäisches Produktzulassungsverfahren, 2003, 152ff.; G. Sydow (Fn. 10), 227; R. Streinz EuR Beiheft 2/2005, 7 (19); dies betont auch die Kommission selbst im Weißbuch Europäisches Regieren, $\operatorname{KOM}(2001)$ 428, 31.

${ }^{134}$ Vgl. V. Mehde (Fn. 27), 54ff.; A. Musil Wettbewerb in der staatlichen Verwaltung, $2005,27 \mathrm{ff}$., der allerdings vielfältige Restriktionen sieht, so dass Wettbewerb v.a. in der mittelbaren Staatsverwaltung oder als benchmarking möglich sei (388); speziell zum Hochschulwesen U. Mager VVDStRL 65 (2006), 274 (288ff.).

135 Vgl. z.B. G. F. Schuppert Verwaltungswissenschaft, 2000, $621 \mathrm{ff}$.

${ }^{136}$ Dazu I. Linsenmann/Ch. Meyer Integration 2002, 285; Th. Bodewig/Th. Voß EuR 2003, 310; J. Lang/K. Burgfeld EuR 2005, 381, die zutreffend ein benchmarking nur zulassen wollen, wenn eine ausdrückliche vertragliche Koordinierungskompetenz der Gemeinschaft besteht (395); E. Szyszczak ELJ 2006, 486; s.a. F. C. Mayer Die Internationalisierung des Verwaltungsrechts, 2005, $343 \mathrm{ff}$. (zit. nach Typoskript). 
reizfaktoren eines Leistungswettbewerbs können eine alternative Form der Output-Legitimation bilden, allerdings nur, wenn sie von einer demokratisch verantwortlichen Stelle und nicht z. B. von der BertelsmannStiftung festgelegt werden. ${ }^{137}$

Die Teilelemente eines solchen pluralistischen Legitimationskonzepts stehen nicht in einem festen Stufenverhältnis und sind auch nicht quantifizierbar. Das komplexe institutionelle Arrangement einer ausdifferenzierten Mehrebenenverwaltung mit einem Mix von Legitimationsmechanismen kann folglich nicht durch eine Exegese verfassungsrechtlicher Normen wie insbesondere des Demokratieprinzips ermittelt werden. Die Schaffung von effektiven Verantwortungsstrukturen ist vielmehr eine Optimierungsaufgabe für den europäischen und den nationalen Gesetzgeber, dem durch die jeweilige konstitutionelle Ordnung nur ein Rahmen vorgegeben ist. 138

Gerade deshalb sind Organisations- und Verfahrensregelungen, mit denen die Rahmenbedingungen des Vollzugs festgelegt werden, wichtige Steuerungs- und Legitimationsinstrumente. ${ }^{139}$ Aus dieser Perspektive bedeutet die neue Formulierung des Art. 84 Abs. 1 GG, die dem Bundesgesetzgeber den Zugriff auf die Behördeneinrichtung und das Verwaltungsverfahren der Länder erschwert, eine bedauerliche Verkürzung von Gestaltungsoptionen. Die damit perpetuierte Vorstellung, man könne die materielle Programmierung von den Parametern Organisation und Verfahren abkoppeln, ist nicht nur in historischer ${ }^{140}$ und rechtsvergleichender ${ }^{141}$ Perspektive einmalig, sie verkennt auch die Bedingungen einer zeitgemäßen Verwaltungspolitik mit einem integrierten Steuerungskonzept. ${ }^{142}$ Die Kompetenzen des Bundesrats sollten anders

137 Vgl. zum föderativen Wettbewerb A. Benz VerwArch 97 (2006), 318 (326ff.); für eine Kompensationsfunktion J.-P. Schneider in: Schmidt-Aßmann/Hoffmann-Riem (Hrsg.) Verwaltungsorganisationsrecht als Steuerungsressource, 1997, 103 (137).

${ }^{138}$ M. Höreth (Fn. 124), 323; B.-O. Bryde in: Redaktion Kritische Justiz (Hrsg.), Demokratie und Grundgesetz, 2000, 59 ff.; G. Lübbe-Wolff VVDStRL 60 (2001), 246 (285); U. Schliesky (Fn. 109), 618 ff.; H.-H. Trute GVwR I, § 6 Rn. 16.

139 C. Franzius GVwR I, § 4 Rn. $50 \mathrm{ff}$.

140 Zur Rechtslage unter der Reichsverfassung von 1871 vgl. R. Mußgnug in: Jeserich/ Pohl/v. Unruh (Hrsg.) Deutsche Verwaltungsgeschichte, Bd. III, 1984, 186 (190f.); zur WRV vgl. H.-P. Bull (Fn. 97), Art. 84 Rn. 2a; a.A. insofern P. Lerche (Fn. 51), Art. 84 Rn. 11; s.a. E. Forsthoff Die öffentliche Körperschaft im Bundesstaat, 1931, 33 ff.

141 Weder die österreichische noch die Schweizer Verfassung kennen vergleichbare Einschränkungen der Regelungsbefugnis des Bundesgesetzgebers; allerdings spricht K. Eichberger in: Ossenbühl (Hrsg.) Föderalismus und Regionalismus in Europa, 1990, 17 (34), von einer „Organisationsautonomie“ der Kantone.

142 Vgl. Th. Groß (Fn. 57), Art. 84 Rn. 26. 
begründet oder strukturelle Alternativen der Länderbeteiligung erwogen werden. ${ }^{143}$

Die nur durch das Subsidiaritätsprinzip begrenzten finalen Kompetenzen des Gemeinschaftsrechts ermöglichen eine breite Palette von Organisationsstrukturen, insbesondere auch durch Formen kooperativer Verwaltung. ${ }^{144}$ In einer polyzentrischen föderalen Ordnung gibt es keinen Vorrang eines bestimmten Legitimationsstranges. Deshalb ist in einer europäisierten Verwaltung die Anerkennung von Entscheidungen „fremder“ Mitgliedstaaten ebenso wenig wie zwischen Bundesländern demokratisch fragwürdig, wenn sie auf einer einheitlichen übergeordneten Rechtsgrundlage beruht und verfahrensrechtliche Mindeststandards gesichert sind. ${ }^{145}$ Dass in einigen Bereichen strukturelle Kontroll- und Verantwortlichkeitslücken festgestellt werden, ${ }^{146}$ ist als Mahnung an den Gemeinschaftsgesetzgeber ernst zu nehmen, mehr Transparenz herzustellen. ${ }^{147}$ Aber auch die Tauglichkeit der nationalen Verwaltungsstrukturen, die ebenso Verflechtungen und sehr unterschiedliche Legitimationsformen kennen, ${ }^{148}$ muss immer wieder überprüft werden. ${ }^{149}$

\section{Fazit}

Das hier skizzierte pluralistische, auf eine Kombination unterschiedlicher Legitimationselemente setzende Konzept reagiert auf die Schwächen des monistischen Modelles, das die Mechanismen einer Mehrebenenverwaltung nicht erfassen kann. Es ist allerdings einzuräumen, dass damit das Ziel der Verantwortungsklarheit ${ }^{150}$ relativiert werden muss. Wenn es keine eindeutigen Zuordnungen von Wahlen, Gesetzgebern und Verwaltungen gibt, sondern gemischte Organisationen und gestufte

143 Vgl. dazu J. Schmidt Die Struktur der zweiten Kammer im Rechtsvergleich, 2006.

144 A. Hatje (Fn. 55), 101.

145 Ähnlich M. Ruffert DV 34 (2001), 453 (480); J. Hecker AöR 127 (2002), 291

(297f.); H.-H. Trute GVwR I, § 6 Rn. 114.

146 E. Grande (Fn. 17), 22; weniger normativ W. Wessels (Fn. 1), 123.

147 A. Peters (Fn. 24), 699; E. Schmidt-Aßmann (Fn. 94), 402; für gestufte Verwaltungsverfahren auch $G$. Winter EuR 2005, 255 (270).

148 B.-O. Bryde VVDStRL 46 (1988), 181; Th. Groß GVwR I, $\$ 13$ Rn. $60 \mathrm{ff}$.

149 Vgl. z.B. zur Neuordnung des Katastrophenschutzes A. Musil/S. Kirchner DV 39 (2006) 373.

${ }_{150}$ Vgl. zu diesem Postulat P. Lerche (Fn. 51), Art. 83 Rn. 110; E. Schmidt-Aßmann (Fn. 94), 399; T. Hebeler (Fn. 56), 50; G. Sydow (Fn. 10), 241; M. Fehling EuR Beiheft 2/2005, 41 (56); H. Ch. Röhl DVB1. 2006, 1070 (1073); H.-H. Trute GVwR I, § 6 Rn. 109; s.a. W. Weiß DV 38 (2005) 517 (542). 
Verfahren als Koordinationsformen der Mehrebenenverwaltung an Bedeutung zunehmen, so dass je nach Aufgabenbereich unterschiedlichen Akteuren Mitwirkungsmöglichkeiten eingeräumt sind, werden die Verhältnisse weniger überschaubar. Diese Unübersichtlichkeit ist aber im Bereich der Verwaltung weniger schädlich als im Bereich der primären Politikgestaltung. Eine generelle Entflechtung als ein Gebot des Demokratieprinzips darzustellen, ${ }^{151}$ geht zu schnell über die sachlichen Vorteile kooperativer Verwaltung hinweg. Nicht jede Form der Verflechtung führt notwendig zu Blockaden, sie kann auch die Problemlösungsfähigkeit erhöhen. ${ }^{152}$ Verantwortungsklarheit ist nur eine Maxime der Organisationsgestaltung, kein präziser rechtlicher Maßstab.

Die Geschichte moderner Verwaltung ist eine Sequenz von Ausdifferenzierungen, die auf die Komplexität der Sachprobleme reagieren, denen sich heutige Gemeinwesen gegenübersehen. Ihre Konsequenzen muss die Verwaltungsrechtsdogmatik verarbeiten, wenn sie nicht durch das Beharren auf Verantwortungskonzepten, die im 19. Jahrhundert entwickelt wurden, den Anschluss an die Wirklichkeit verlieren will. Die demokratische Verantwortlichkeit von Herrschaftsgewalt kann nicht allein durch die Einrichtung einer bestimmten, historisch kontingenten Verwaltungsorganisation garantiert werden, sie muss vielmehr durch variable Regelungsstrukturen, die an unterschiedliche Ebenen und Aufgaben angepasst sind, immer wieder neu aktualisiert werden.

151 P. M. Huber 65. DJT, 2004, D $33 \mathrm{ff}$.

152 A. Benz PVS 1998, 558 (560); F. W. Scharpf Regieren in Europa, 1999, 109; A. Peters (Fn. 24), 189. 
Leitsätze des 2. Berichterstatters über:

\section{Verantwortung und Effizienz in der Mehrebenenverwaltung}

\section{Die polyzentrische Struktur der Mehrebenenverwaltung}

(1) Die drei terminologischen Angebote Mehrebenensystem, Netzwerk und Verwaltungsverbund weisen auf zutreffende Aspekte der europäisierten Verwaltung hin, für die Lösung konkreter Rechtsfragen im Verhältnis zwischen den Ebenen leisten sie jedoch keinen Beitrag.

(2) Durch die Integration eines Bundesstaates in die Europäische Gemeinschaft entsteht ein doppelter Föderalismus, in dem drei Ebenen der Gesetzgebung und drei Ebenen der Verwaltung - unter Einbeziehung der kommunalen Ebene sogar vier - miteinander kombiniert werden.

(3) Auf allen vier Ebenen bestehen politisch selbständig legitimierte Verwaltungen, deren Koordination durch verfassungsrechtliche und gesetzliche Regelungen organisiert wird, bei der gleichzeitig aber auch die Autonomie der unteren Ebenen respektiert werden muss, so dass sich ein polyzentrisches System bildet.

II. Die Regeln der Aufgabenverteilung

1. Formen der Aufgabenverteilung

(4) Neben dem dezentralen Gesetzesvollzug und der reinen Zentralverwaltung gibt es zwei Mischformen: die Zentralverwaltung unter Mitwirkung von Vertretern der dezentralen Ebene und die gemeinsame Verwaltung.

\section{Allgemeine Rechtsgrundsätze der Aufgabenverteilung}

(5) Das Subsidiaritätsprinzip ist auf den administrativen Vollzug des Gemeinschaftsrechts ohne weiteres anwendbar, aber es eröffnet dem Gemeinschaftsgesetzgeber einen politischen Einschätzungsspielraum bezüglich der Notwendigkeit einer Zentralisierung von Verwaltungskompetenzen.

(6) Dem Grundgesetz lässt sich kein ungeschriebener Grundsatz entnehmen, wonach die dezentrale Ebene vorzuziehen und die Zentralisierung von Verwaltungsaufgaben deshalb begründungsbedürftig ist. 
(7) Das deutsche Recht begrenzt Mischverwaltung durch den Verfassungsvorbehalt des Art. 83 GG, kennt aber kein konsequentes Gebot der Ebenentrennung.

(8) Im primären Gemeinschaftsrecht fehlen ausdrückliche Begrenzungen für Kooperationsverhältnisse, da der Rat als Vertretung der Mitgliedstaaten in die Durchführung des Gemeinschaftsrechts einbezogen werden kann.

\section{Die Koordination der Ebenen}

\section{Die Typen der vertikalen Koordination}

(9) Mehrere Verwaltungsebenen können durch sechs Typen von Instrumenten koordiniert werden: Informationsaustausch, Rechtskontrolle, abstrakt-generelle Normkonkretisierung, Einzelweisung, Übernahme der Zuständigkeit und gemeinsame Entscheidungsverfahren.

\section{Die Durchsetzungsmechanismen}

(10) Im Allgemeinen sind administrative Durchgriffsmöglichkeiten auf Einzelentscheidungen in einem föderalen Verhältnis ausgeschlossen.

(11) In Mehrebenensystemen ist eine übergreifende Personalgewalt nur ganz ausnahmsweise vorgesehen.

(12) Eine Ermächtigung zu einem Gewalteinsatz gegen föderale Gliedstaaten ist in einer modernen Verfassungsordnung überflüssig.

(13) Neben den exekutiven Mechanismen gibt es weitere Instrumente, mit denen die Durchsetzung von Rechtsnormen durch externe Kontrollen gefördert werden kann.

\section{Die Schaffung von Legitimation als Gestaltungsaufgabe}

1. Die Verantwortungsverteilung im Mehrebenensystem

(14) Die polyzentrische Mehrebenenverwaltung führt notwendig zu einer Verteilung der Verantwortung, die mit dem traditionellen monistischen Modell der Verwaltungslegitimation in Konflikt gerät.

\section{Das institutionelle Legitimationsarrangement}

(15) Ein realitätsgerechtes Modell muss von dem Pluralismus der Legitimationsquellen ausgehen, in dem viele unterschiedliche Akteure an einem arbeitsteiligen Implementationsprozess beteiligt sind. 
(16) Wichtige Legitimationsinstrumente sind Transparenz und Partizipation, die in erster Linie den organisierten Interessen, aber auch der breiten Öffentlichkeit die Möglichkeit der Mitwirkung an Entscheidungsprozessen bieten.

(17) Das Modell entpolitisierter Fachverwaltung eignet sich für Aufgabengebiete, in denen nach weitgehend objektivierbaren Effizienzkriterien entschieden werden kann.

(18) Die Zielvorgaben und Anreizfaktoren eines staatlich geschaffenen Wettbewerbs in der Verwaltung können eine alternative Form der OutputLegitimation bilden.

\section{Fazit}

(19) Verantwortungsklarheit ist nur eine Maxime der Organisationsgestaltung, kein präziser rechtlicher Maßstab.

(20) Die demokratische Verantwortlichkeit von Herrschaftsgewalt kann nicht allein durch die Einrichtung einer bestimmten, historisch kontingenten Verwaltungsorganisation garantiert werden, sie muss vielmehr durch variable Regelungsstrukturen, die an unterschiedliche Ebenen und Aufgaben angepasst sind, immer wieder neu aktualisiert werden. 


\section{Aussprache und Schlussworte}

\section{Verantwortung und Effizienz in der Mehrebenenverwaltung}

Diskussionsleiter (F. Kirchhof): Wir dürfen mit der Diskussion beginnen und folgen nach altem Brauch der Uhr, die uns begleitet, ermahnt und irgendwann rot zeigt; dann schweigen wir. Wenn wir eine Hitliste der vier Gliederungspunkte aufstellen würden, erhielte der Themenblock „Legitimation“ eindeutig Platz 1.

Ohler: Ich möchte nur einige wenige Anmerkungen zum Themenkomplex 1 machen. Herr Groß erwähnte die Frage der gemeinschaftlichen Rechtsetzungskompetenz für die Verwaltungsorganisation auf Gemeinschaftsebene und auf Ebene der Mitgliedstaaten. Zu Recht ist in diesem Zusammenhang das Urteil des Europäischen Gerichtshofs in der Rs. C-217/04 (ENISA) erwähnt worden. Ich habe dabei aber die Befürchtung, dass man mit Hilfe der Annexkompetenzen bzw. von implied powers ein zu großes Problem stemmen möchte. Zur Erinnerung: Der Europäische Gerichtshof hat die Zuständigkeit der Gemeinschaft zur Erschaffung einer EG Agentur auf implied powers in Verbindung mit Art. 95 EGV gestützt. Wir haben bei den Vorträgen jedoch erahnen dürfen, welche rechtliche und politische Komplexität sich hinter dem Thema Verwaltungsorganisation verbirgt. Dass wir diese Komplexität mit dem Instrument der implied powers hebeln können, erscheint mir außerordentlich fraglich.

Einige Bemerkungen möchte ich zu den Begriffen Verantwortung und Effizienz in der Mehrebenenverwaltung hinzufügen. Beide Termini sind zweifelsohne auch in der juristischen Diskussion sehr beliebt und weisen den Vorzug der Anschaulichkeit auf. Dennoch habe ich immer Sorge, wie handhabbar sie in der rechtlichen Realität sind. Ich ziehe es daher vor, zumindest den Begriff der Verantwortung gegen den Rechtsbegriff der Zuständigkeit zu tauschen und möchte anhand von verschiedenen Urteilen des EuGH nachweisen, dass Fragen der internationalen Zuständigkeit, um die es im Mehrebenensystem immer geht, durchaus schwierige Probleme nach sich ziehen. Der erste Bereich betrifft die internationale Zuständigkeit innerhalb der EU zur Erteilung von Führerscheinen. Die Führerscheinrichtlinie 91/439 knüpft hier an das Herkunftslandsprinzip an, d.h. verleiht dem Mitgliedstaat die Zuständigkeit, 
in dem eine Person ihren Wohnsitz hat. Was passiert aber, wenn der Person in diesem Mitgliedstaat der Führerschein entzogen wird? In der Praxis hat sich leider ein illegaler Führerscheintourismus entwickelt, bei dem Personen, die in Deutschland den Führerschein verlieren, in einem anderen Mitgliedstaat, der es, um es vorsichtig zu formulieren, vielleicht nicht so genau nimmt, einen neuen Führerschein erwerben. Dieser ausländische Führerschein darf nach der ständigen Rechtsprechung des EuGH, beginnend mit der Entscheidung Kapper, in Deutschland nicht entzogen werden. Der Europäische Gerichtshof begründet das mit der Pflicht zur gegenseitigen Anerkennung und der alleinigen Zuständigkeit des ausstellenden Mitgliedstaats, diesen Führerschein wieder zu entziehen. Selbst der Verstoß gegen die internationale Zuständigkeit, aufgrund dessen es überhaupt nur zu der Erteilung des Führerscheins kam, beeindruckt den EuGH nicht. Ein völlig anderer Gesichtspunkt zum Themenkreis internationale Zuständigkeit ergibt sich aus der Entscheidung Egenberger des Europäischen Gerichtshofes. Hier ging es um die Erteilung von Einfuhrlizenzen für verbilligte neuseeländische Butter. Die ausschließliche internationale Zuständigkeit für die Erteilung dieser Lizenzen lag aufgrund der einschlägigen Verordnung der EG bei einer englischen Behörde. Hiergegen richtete sich die erfolgreiche Klage der Firma Egenberger. Der EuGH hob die Zuständigkeitsregel mit der Begründung auf, sie stelle einen Verstoß gegen den allgemeinen Gleichheitssatz dar, weil sie Wirtschaftsteilnehmer aus anderen Mitgliedstaaten benachteilige. Wir haben es also in beiden von mir gestreiften Bereichen mit Fragen der internationalen Zuständigkeit vor dem Hintergrund der Binnenmarktintegration zu tun, die aber zu völlig verschiedenen Antworten führen. Wie wir hierzu dann noch das Thema Verwaltungseffizienz in Stellung bringen, lässt sich nur im Einzelfall beantworten. Auf die möglichen Konflikte hat ja dann Herr Pache ausführlich hingewiesen.

Huber: Ich wollte drei kurze Bemerkungen zum Referat von Herrn Groß machen. Das Erste: Sie haben den Bundeszwang verabschiedet. Nachdem wir nun 50 Jahre „Schönwetterperioden“ hinter uns haben und auch die europäische Rechtsgemeinschaft unter den 15 alten Mitgliedern einigermaßen funktioniert hat, würde ich dem als empirischer Bestandsaufnahme nicht widersprechen. Man hat den Bundeszwang in den letzten 60 Jahren in der Tat nicht gebraucht. Aber wer gibt uns die Garantie, dass es in 10 Jahren nicht anders sein wird? Ich könnte mir nicht nur das gegenwärtige Zwillingspaar in Polen, sondern auch andere Politiker in Europa vorstellen, die es mit den Spielregeln der Rechtsgemeinschaft so genau nicht nehmen. Ich könnte mir sogar renitente (Bundes-)Länder vorstellen, gegenüber denen man den Bundeszwang unter 
Umständen noch brauchen wird - natürlich nicht den Freistaat Bayern. Vor diesem Hintergrund scheint es mir - sagen wir mal - ein bisschen rosarot gemalt, auf dieses Instrument verzichten zu wollen. Das Zweite: als empirischer Befund ist die Komplexität der Verwaltungskooperation, die Sie geschildert haben, realistisch beschrieben, richtig und nicht $\mathrm{zu}$ bezweifeln. Aber wenn das Ergebnis ist, dass Verantwortung - und das war, wenn ich Sie richtig verstanden habe, ja Ihre Schlussthese - unter den Bedingungen von Europäisierung und Globalisierung notgedrungen verunklart wird, dass ein Preis in Einbußen an demokratischem Legitimationsniveau zu zahlen ist, dann stelle ich mir die Frage, ob wir als Rechtswissenschaftler - anders als Politologen und Historiker - bei diesem Befund stehen bleiben können oder ob nicht ein Rechtsprinzip wie die Demokratie auch normative Vorgaben enthält, die uns dazu verpflichten, als Staatsrechtslehrer darüber nachzudenken, ob man nicht ein gewisses Maß an Effizienzverlust und Komplexitätsreduzierung in Kauf nehmen muss, um die notwendige Verantwortung, die die demokratische Abstützung unserer Gemeinwesen auf allen vier Ebenen erst möglich macht, sicherzustellen. Und die dritte Bemerkung: Ich glaube, Art. 84 GG haben Sie falsch verstanden und die Föderalismusreform insoweit zu Unrecht gescholten. Der Art. 84 GG n. F. hat den Zweck, dem Bund den Erlass von Verfahrensregelungen zu erleichtern. Das Zustimmungserfordernis ist weggefallen, weil man - und Herr Grimm und Herr Meyer haben schon in der Bundesstaatskommission wie Löwen für diese Einsicht gekämpft - materielles Recht und Verfahrensrecht nicht trennen kann. Deshalb wollte man für den Bund die Möglichkeit erleichtern, hier zu legeferieren und das Risiko mindern, am Widerstand des Bundesrates zu scheitern. Die zweite Variante ist die Beibehaltung des Status quo. Deshalb ist die Vorschrift gerade im Lichte Ihres Anliegens, glaube ich, eine Verbesserung und keine Verschlechterung.

Häde: Ich möchte anknüpfen an die These 6 von Herrn Groß. Dass sich dem Grundgesetz kein ungeschriebener Grundsatz entnehmen lässt, wonach die dezentrale Ebene vorzuziehen sei, ist sicher richtig. Und Herr Groß hat seine These zusätzlich noch unter den Vorbehalt gestellt, dass natürlich das Grundgesetz selber gewisse Regeln vorsieht, die gelten, wenn der Bund sich zusätzliche Verwaltungskompetenzen schaffen will. Das war aber mehr eine Art Nebensatz und ich möchte gerade das noch einmal betonen. Es gibt eine Verfassungsgrundentscheidung für die dezentrale Ausführung, für die dezentrale Verwaltung. Daraus folgen sicherlich keine zusätzlichen materiell-rechtlichen Anforderungen, aber immerhin die verfahrensrechtliche Anforderung, dass der Bund seine Verwaltungszuständigkeiten nur durch Bundesgesetz erweitern darf. 
Und das kann er auch nur in dem Rahmen, den Art. 87 Abs. 3 GG vorsieht, also grundsätzlich nur durch die Errichtung von Bundesoberbehörden, Körperschaften oder Anstalten ohne eigenen Verwaltungsunterbau, soweit nicht Art. 87 Abs. 3 Satz 2 GG Ausnahmen zulässt. Diese Voraussetzungen sind wichtig und bringen die Verfassungsgrundentscheidung für die dezentrale Ausführung in einer Weise zum Ausdruck, dass man sie schon etwas stärker betonen sollte. Eine zweite Bemerkung zu These 12. Auch Herr Huber hat gerade schon daran angeknüpft. Herr Groß hält den Bundeszwang als Gewalteinsatz gegen die Länder für überflüssig. Das mag in der Praxis bisher so gewesen sein. Immerhin haben die wissenschaftlichen Dienste des Deutschen Bundestages aber vor kurzem eine Ausarbeitung herausgegeben, die auf eine Anfrage des Vorsitzenden des Haushaltsausschusses des Bundestages zurückgeht. Es geht um die Frage, ob man für Berlin einen Sparkommissar einsetzen kann. Das ist eine sehr aktuelle Problematik. Ich denke nicht, dass das in diesem konkreten Fall ein geeignetes Instrument wäre. Aber diese Überlegungen zeigen doch, dass der Art. 37 in der aktuellen Politik nicht als überflüssig betrachtet wird. Eine letzte Bemerkung noch zu den Vorzügen des Föderalismus allgemein. Es geht aus meiner Sicht nicht nur darum, dass man bürgernäher handelt oder dass damit sonstige Vorteile verbunden sind. Ein Argument wird häufig vernachlässigt: Es geht auch darum, dass man auf diese Weise verhindert, dass jeder Fehler gleich bundesweit gemacht wird. Allein wenn man an die Schulpolitik denkt, ist das ein großer Vorzug des Föderalismus.

P. Kirchhof: Ich glaube, wir haben zwei eindrucksvolle Referate gehört, die gerade im europäischen Verbund bewusst machen, was sich ereignet an Kooperation, an Verflechtung, an Zusammenwirken. Meine Frage geht dahin, wo ist die Beunruhigung der Referenten? Wo ist das Alarmsignal? Es muss doch alles letztlich dem Bürger dienen. Und den Wählern. Und wenn der Wähler nicht erkennen kann, wer letztlich verantwortlich ist, wen soll er wählen und abwählen? Wenn der Bürger ein Anliegen hat, und er weiß nicht, soll ich mich an ein Bundesland wenden oder nach Berlin oder nach Brüssel, dann nehme ich ihm real Rechte. Wenn was schief gelaufen ist, wer haftet? Wen soll ich verklagen? Bei welchem Gericht? Wer prüft? Bundesrechnungshof? Landesrechnungshof? Europäischer Rechnungshof? Ich meine, wir sollten diese eindrucksvolle Analyse weiterdenken mit der Frage, ob hier nicht dem Staat, dem klassischen Staat, eine besondere Verantwortlichkeit zuwächst, nämlich all dies Recht aus verschiedenen Quellen dem Bürger gegenüber als das alleinige Organ, mit dem der Bürger zu tun hat, zu vertreten, zu erklären und letztlich auch zu verantworten. Also erwächst 
aus Ihrer Analyse eine sehr moderne und sehr aktuelle neue Staatsaufgabe. Zweiter Punkt: Kooperation ist die Verhandlungslösung, und die Verhandlungslösung hat immer etwas Kompromisshaftes. Und ist nicht gerade die Schwäche unserer Systeme EG und Staat heute das Kompromisshafte? Die Demokratie braucht den Kompromiss, aber die Demokratie wird sterben, wenn die Demokraten an dem Kompromiss nicht mehr leiden. Ich glaube das ist genau das Thema unserer heutigen Diskussion. Und der dritte und letzte kurze Punkt. Wenn wir die Verhältnismäßigkeit, Herr Pache, und das Effizienzprinzip definieren, wie Sie es getan haben, geringstmöglicher Mitteleinsatz zu größtmöglichem Erfolg, also optimierungs-superlative Tatbestände, dann verschieben Sie die Kompetenz immer zum Richter. Wenn der Richter über etwas entscheidet, ein Gesetz, einen Verwaltungsakt, tut er es in der Distanz des gelassenen Verfahrens eines Senats, der alles beobachten kann, besonders welche Fehler gemacht worden sind und im Verzicht auf das Kompromisshafte der Politik. Dann weiß der Richter immer etwas Besseres. Optimierungsstrategien sagen dem Richter „denk dir etwas aus und wenn du klüger bist, weißt du es besser“. Und das macht unsere gesamte Kompetenzordnung kaputt. Deswegen meine Frage, müssen wir nicht sagen: Effizienz und Verhältnismäßigkeit sind der angemessene Einsatz von Mitteln zu dem erstrebten Zweck. Dann haben wir Respekt vor der Gesetzgebungsverantwortung, Respekt vor der Exekutivverantwortung und nicht ein überschießenden Element des das Beste Wollens, was letztlich die Kompetenz des Richters ist.

Hermes: In erfreulicher und deutlicher Übereinstimmung haben beide Referate das monistische Modell demokratischer Legitimation moderner Verwaltung verabschiedet und haben diese Verabschiedung nach meiner Einschätzung mit einer eindrucksvollen Darstellung und Analyse der modernen ausdifferenzierten polyzentrischen Verwaltung belegt. Sie haben dabei nicht - etwa in dem Sinne von Herrn Huber - die Realanalyse sozusagen eins zu eins normativ umgesetzt, sondern dafür plädiert, das Demokratieprinzip in einem modernen Sinne weiter zu entwickeln. Deshalb würde ich mir abweichend von Herrn Häberle wünschen, von Rostock möge das Signal ausgehen, daß die Deutsche Staatsrechtslehrervereinigung das monistische Legitimationsmodell jedenfalls was die polyzentrischen modernen Verwaltungsstrukturen angeht - verabschiedet hat. Diese grundsätzliche Zustimmung möchte ich allerdings um zweifelnde Nachfragen ergänzen. Diese Zweifel gehen durchaus in die Richtung, die Herr Huber bereits angedeutet hat. Offen ist nämlich, ob mit der übereinstimmenden Verabschiedung des traditionellen Legitimationsmodells bereits ausreichende Antworten auf die 
sich dann anschließenden Fragen gefunden sind. Wenn ich es richtig sehe, hat Herr Pache die Antwort vor allem in dem Prinzip gesucht, wonach der Gesetzgeber - unabhängig davon, ob auf europäischer oder auf nationaler Ebene - die Verantwortung im Sinne eines Optimierungsgebotes dafür trägt, die verschiedenen Legitimationsbausteine entsprechend dem jeweiligen Sachbereich neu zuzuordnen und zu einem vernünftigen Gesamtkonzept zusammenzustellen. Herr Groß ist ein Stück weiter gegangen und hat uns noch einzelne Modelle für verschiedene Verwaltungsaufgabentypen vorgestellt - insbesondere das Modell der entpolitisierten Fachverwaltung. Meine sich daran anschließende Frage, die sich an beide Referenten richtet: Sind wir schon an dem neuen Ufer angekommen oder befinden wir uns noch mitten auf dem Fluß? Mein Eindruck ist, das neue Ufer ist noch nicht erreicht. Konkret am Beispiel der These 17 von Thomas Groß: Da wird vorgeschlagen, das Modell entpolitisierter Fachverwaltung eigne sich für Aufgabengebiete, in denen nach weitgehend objektivierbaren Effizienzkriterien entschieden werden kann. Daran schloss sich dann allerdings eine Einschränkung an. Wenn es sich nämlich um Entscheidungen handele, die auf den ersten Blick technokratischen Charakter haben, die auf den zweiten Blick aber mit erheblichen Wertungsspielräumen verbunden sind, dann müsse - bezogen auf die europäische Ebene - doch die Kommission entscheiden. Dann frage ich mich, wo diese entpolitisierten Fachverwaltungsentscheidungen eigentlich anzusiedeln sind. Wenn man sich das Lebensmittelrecht oder das technische Sicherheitsrecht z. B. im Eisenbahnwesen und in vielen anderen Sektoren näher anschaut, dann zeigen sich hinter allen diesen Standards und Einzelentscheidungen erhebliche Spielräume und letztlich politische Wertungen. Wo bleibt dann der Anwendungsbereich des Modells entpolitisierter Fachverwaltungen? Sollte man nicht darüber nachdenken, Organisations- und Verfahrensstrukturen zu entwickeln, die im „Normalfall“" technokratische Fachverwaltungen nach Effizienzkriterien entscheiden lassen, die aber die Möglichkeit vorsehen, in Fällen mit erhöhtem Konfliktpotential die konkrete Sachentscheidung wieder nach oben in die politische Instanz zu heben. Als Orientierungsbeispiele könnten aus dem Wettbewerbsrecht die Ministergenehmigung oder aus dem Regulierungsrecht die zu veröffentlichende Weisung des Ministeriums gegenüber der Bundesnetzagentur dienen.

Pernice: Erlauben Sie mir eine kleine Ergänzung zum Referat von Herrn Pache: Zum Grundsatz der Organisations- und Verfahrenautonomie der Mitgliedstaaten, also der Idee, dass die Mitgliedstaaten vorzüglich kompetent sind, Europarecht umzusetzen und durchzuführen, kann man auch auf die Erklärung Nr. 43 zum Subsidiaritätsprotokoll verweisen, 
vielleicht auch auf eine neue Entscheidung des EuGH vom 19. September 2006 (Rs. C-392/04 und C-422/04), wo dieser Grundsatz in der Rechtsprechung wohl erstmalig ausdrücklich genannt wird. Mein Thema ist aber die Legitimationsfrage, und ich möchte zu den sehr ausführlichen aber relativ abstrakten Referaten ein Beispiel aus dem Telekommunikationsrecht einführen. Dort ist für bestimmte Fragen der Marktabgrenzung in den Richtlinien und dann auch in den entsprechenden Gesetzen vorgesehen, dass die Stellungnahmen der Regulierungsbehörden anderer Mitgliedstaaten maßgeblich von der jeweiligen Regulierungsbehörde eines Mitgliedstaates zu berücksichtigen sind. Sie besitzen also Maßstabswirkung, wenn auch vielleicht nicht volle Bindungswirkung. Aber es geht so weit, dass etwa die Bundesnetzagentur bei uns dann nicht autonom entscheidet. Daraus ergibt sich dann die Frage, wie im Falle einer derartigen europaweiten Koordinierung für die betreffende Verwaltungsentscheidung zwei Dinge nach klassischem Muster weiter garantiert werden können. Erstens: Die Legitimation der Entscheidung vermittelt durch die Legitimation des Ministers als Teil der gewählten Regierung. Kann ich dem Minister überhaupt das Weisungsrecht geben in einem solchen Fall, wenn er bei der Koordination doch nicht teilgenommen hat und die Behörde sogar an die Stellungnahmen der anderen nationalen Regulierungsbehörden gebunden ist? Hier würde ich sagen: Es gibt einen Spielraum der Unvertretbarkeit, der durch ministerielle Weisungen jedenfalls weder beschränkt noch ersetzt werden kann. Die zweite Frage ist der Rechtschutz: Kann ich einem deutschen Gericht die Kompetenz geben, darüber zu urteilen, ob diese Entscheidung, die kooperativ im europäischen Koordinierungsverfahren erstellt worden ist, rechtmäßig ist oder nicht? Ich glaube in Bezug auf das Verfahren fällt die Antwort positiv aus. Aber in materiellrechtlicher Hinsicht würde ich dafür plädieren, dass eine Unvertretbarkeit der Entscheidung insofern anzunehmen ist, als auch der Richter an dieser Koordinierung nicht teilgenommen hat. Er weiß also nicht, welches die Erwägungen der anderen Regulierungsbehörden bei der Erarbeitung der gemeinsamen Entscheidung waren. Die Quintessenz daraus ist, dass nur ein begrenzter Rechtschutz gegeben sein kann, und auch nur eine begrenzte ministerielle Verantwortung. Wenn man traditionell Legitimation nach dem „Kettenmodell“, dh. aus der Legitimationskette vom Bürger her begründet, lautet meine Frage jetzt an beide Referenten: Wie kann ich dem Problem des Legitimitäts- und Rechtschutzdefizits entrinnen? Ich habe bei Ihnen herausgehört, Herr Pache, dass sie eine gesetzgeberische Lösung vorschlagen. Also Sie berufen sich darauf, dass der Gemeinschaftsgesetzgeber entsprechende Lösungen erarbeiten müsste. Ich habe allerdings bisher nicht viel Konkretes dazu gehört, wie das aus- 
sehen könnte. Denken Sie dabei eventuell in punkto Rechtschutz an ein europäisches Verwaltungsgericht, was dann solche Entscheidungen überprüfen müsste? Aber auch da wäre das Problem der Unvertretbarkeit ja gegeben.

Hoffmann-Riem: Ich begrüße es sehr, dass die beiden Referenten das Legitimationskonzept weit gezogen, nach einer Vielfalt von Legitimationsbausteinen gesucht und dabei Möglichkeiten einer output-orientierte Legitimation mit eingebaut haben. Ich habe allerdings vermisst, dass das Denken über Legitimation in verschiedenen Ebenen auch noch auf eine weitere, gewissermaßen eine fünfte, Ebene erstreckt wird, nämlich auf den Bereich der Kooperation mit Privaten oder auf die Übertragung von öffentlichen Aufgaben an Private. Hier stellt sich das Legitimationsproblem viel schwieriger als in der von den Referenten behandelten staatlichen bzw. europäischen Mehrebenenverwaltung. Wenn man nach Möglichkeiten einer Legitimation auch für diesen Bereich sucht, muss man sicherlich an die Vielfalt der erwähnten Legitimationsbausteine denken, aber vermutlich noch manche andere einbeziehen. Eine zweite Bemerkung knüpft an den Punkt 4, die Bedeutung von Effizienz, an. Der Vorstand und offenbar auch die Referenten - dem stimme ich zu - haben Effizienz als einen Rechtsbegriff verstanden und versucht zu zeigen, wie er operationalisiert und auf die Verwaltung bezogen werden kann. Man sollte Effizienz als die angemessene Relation zwischen Aufwand und Erfolg aber nicht nur auf den Ressourceneinsatz der Verwaltung beziehen, sondern auch fragen, wie es um die Effizienz aus der Sicht der von der gefundenen Lösung Betroffenen steht: Wie sieht die Relation von Aufwand und Nutzen für sie - für das, was von ihnen erwartet wird oder ihnen zugemutet wird - aus? Gelegentlich besteht nämlich die Gefahr, dass beide Effizienzperspektiven auseinander fallen. Nur, wenn die Effizienz des Verwaltens sich auch in der Art der Bewältigung des administrativ zu lösenden Problems fortsetzt, ist das geleistet, was in einem Rechtsstaat zu leisten ist, also insbesondere den Verhältnismäßigkeitsgrundsatz zu beachten. Es geht niemals um die Verwaltung als Selbstzweck, sondern immer um ihren Beitrag zur sachgerechten Aufgabenerfüllung. Wenn eine solche Effizienz bei der Problembewältigung auch für die betroffenen Bürger gesichert ist, kann der Bezug zum Legitimationsproblem leichter hergestellt werden: Eine so verstandene Effizienz leistet auch einen Beitrag zur output-orientierten Legitimation.

Jestaedt: Ich könnte meine Bemerkungen in dem Satz zusammenfassen: Die Botschaft hör' ich wohl, allein mir fehl'n die Normen. Meine Frage geht also dahin, ob Sie beide den Positivierungsnachweis für die von Ih- 
nen behaupteten konkreten Anforderungen demokratischer Legitimation führen können.

Dabei darf ich vorwegschicken, dass ich Sie so verstanden habe, dass, wenn Sie von demokratischer Legitimation sprechen, Sie vom geltenden Recht und den von ihm aufgerichteten Rechtmäßigkeitsbedingungen handeln und nicht lediglich von einem verfassungs theoretischen Konzept von demokratischer Legitimation oder gar bloßer demokratischer Legitimität. Sie, Herr Pache, haben zum Beispiel gesprochen über die demokratischen Vorgaben des europäischen Verfassungsverbundes für Verwalten; ich nehme an, dass Sie damit auch und gerade positivrechtliche Vorgaben in Bezug genommen haben. Andernfalls, das heißt bei bloß verfassungstheoretischem Räsonnement, hätten wir es mit etwas letztlich Zahnlosem, nämlich mit bloßer Theorie und nicht mit geltendem Recht, zu tun. Meinen Fragen liegt daher die Prämisse zugrunde, dass Sie beide über die demokratisch-legitimatorischen Anforderungen räsoniert haben, die das geltende Recht aufstellt.

Dies vorausgeschickt, frage ich Sie: Wo ist Ihrer Meinung nach im Gemeinschaftsrecht, ich muss ja wohl sagen: im Gemeinschaftsprimärrecht, das demokratische Prinzip mit seinen Legitimationsanforderungen niedergelegt? Wo situieren Sie das gemeinschaftsrechtliche Demokratieprinzip? Aus meiner Sicht geht es, wenn wir über das geltende Recht reden, gar nicht darum, ob wir persönlich ein traditionelles monistisches Legitimationskonzept oder aber ein - dann ja wohl: fortschrittliches pluralistisches Legitimationskonzept für vorzugswürdig halten und uns dafür stark machen, sondern zunächst schlicht und ergreifend darum, für welches Konzept sich die positivrechtlichen Grundlagen nachweisen lassen. Einen Positivisten wie mich haben Sie sofort auf Ihrer, für ein pluralistisches Legitimationskonzept optierenden Seite, wenn Sie nur die entsprechenden Normen anführen können. Die Frage, die sich insoweit zunächst stellt: Wer ist nach dem geltenden Gemeinschaftsrecht das demokratische Legitimationssubjekt? Danach entscheidet sich, ob das Legitimationskonzept des positiven Rechts monistisch oder pluralistisch ausgelegt ist. Sodann: Was ist aus Sicht des Gemeinschaftsrechts das demokratische Legitimationsobjekt? Was muss, mit anderen Worten, überhaupt legitimiert werden? Und die wichtigste Frage, um deren Beantwortung Sie die ganze Zeit herumgeschlichen sind: Was ist und wie bestimmt man das demokratische Legitimationsniveau? Welches sind die unterschiedlichen Legitimationsmodi? Wie können sie wechselseitig in Beziehung gesetzt werden? Und wie ist das Maß oder die Höhe dessen zu bestimmen, was als Legitimationsniveau bezeichnet wird? Wer keine Angaben dazu macht, wie sich das nach geltendem Recht geforderte Legitimationsniveau bestimmt, der kann auch nicht berechtigter- 
weise davon sprechen, dass das erforderliche Niveau demokratischer Legitimation in dem einen oder anderen Fall unterschritten oder auch erreicht sei. Damit fehlt jeder Anhaltspunkt dafür, wann die (gemeinschafts)verfassungsrechtlichen Anforderungen eingehalten und wann sie verletzt sind. Sie sprechen in diesem Zusammenhang von der Legitimationsrelevanz von Verfahren, von Transparenz, von input- und outputLegitimierung. Das alles ist nicht implausibel. Ich frage mich aber: Wo findet sich das im positiven Recht? Wie bestimmt und berechnet sich das Legitimationsniveau? Wer davon spricht, dass ein konkretes Niveau demokratischer Rückführbarkeit erreicht werden müsse, behauptet Maßstäbe, an denen gemessen werden kann, behauptet rechtlich Berechenbares. Und wenn Sie insinuieren, dass einzelne Legitimationselemente durch andere substituiert werden können, dann rechnen Sie offensichtlich auch. Dann aber interessiert mich: Welches sind die demokratisch-legitimatorischen Grundrechenarten, mit denen Sie operieren, und woher haben Sie die? Eine weitere Frage: Woraus ergibt sich, dass das demokratische Prinzip ein Optimierungsgebot ist? Warum ist es keine Regel? Wenn ich das Grundgesetz zur Hand nehme - ich spreche jetzt fürs deutsche Verfassungsrecht, für das europäische Recht müssten Sie den Nachweis noch liefern -, so lese ich, dass alle Staatsgewalt ohne jeden Vorbehalt vom Volke ausgehen müsse. In Art. 20 Abs. 2 Satz 1 GG steht nicht, dass nach Möglichkeit alle Staatsgewalt vom Volke ausgehen solle. Ich weiß infolgedessen nicht, womit Sie die These, das Demokratieprinzip sei ein bloßes Optimierungsprinzip, belegen wollen. Eine andere Frage: Welche Rechtsfolge tritt ein, wenn die Legitimationsanforderungen einmal nicht erfüllt sind? Nach Ihren Ausführungen war alles so harmonisch, dass sich der Fall des Unterschreitens des Legitimationsniveaus offenbar niemals einstellt. Ist dann das Prinzip demokratischer Legitimation ein zahnloser Tiger? Damit bin ich bei meiner letzten Frage: Könnte es vielleicht sein, dass die Bezugnahme auf das Legitimationsniveau Ihnen nur dazu dient, rechtspolitische Forderungen demokratietheoretisch in ansprechender Weise zu drapieren?

Häberle: Darf ich die in unserem Kreis einst so reiche Tradition der „Spontanbeiträge“ wiederbeleben? Ihre klassische Zeit war die eines Günter Dürig, auch die in der großen Zeit von Hans Meyer, der uns immer wieder mit Spontanbeiträgen provozierte und forderte. Darum jetzt ein Spontanbeitrag zu Ihnen, Herr Jestaedt, und eine „Nachlieferung“ zu Ihnen, Herr Kirchhof. Wir können doch nicht mehr behaupten, im europäisierten Deutschland gehe alle Staatsgewalt vom Volk aus, sondern wir müssen formulieren: Alle Staatsgewalt geht auch vom deutschen Volk aus und sie ist im Übrigen „europäisiert“. Wir können nicht 
mehr mit dem Vokabular klassischer Allgemeiner Staatslehre arbeiten, wie Sie dies tun, werter Herr Kirchhof, indem Sie sagten, der Staat habe die Aufgabe dem Bürger klarzumachen, wo die Verantwortungen liegen. Wir haben doch nicht nur den deutschen Bürger, sondern auch die Gesamtheit der Unionsbürger. Es gibt die Europawahlen, und in ihnen können wir sehr wohl bestimmte Leute, die EU-Abgeordneten, abwählen und in Verantwortung nehmen. Sie, meine Damen und Herren, werden einwenden, dies sei alles Idealismus, aber ein Stück Idealismus und Optimismus braucht man in Verfassungsdingen, in der Politik, in Bürger- und Menschheitsfragen. - Hier eine letzte Fußnote. Ich möchte eine Brücke schlagen, wohl ganz im Sinne des Vorstandes, zwischen den Themen des heutigen Vormittages und Nachmittages. Müsste es uns nicht gelingen, im Geiste des großen Fritz Werner sein Dictum vom „Verwaltungsrecht als konkretisiertes Verfassungsrecht“ zu übertragen? Können wir nicht angesichts der beiden sehr eindrucksvollen verwaltungsrechtlichen Referate eine gewisse Konvergenz zwischen dem heutigen Vormittag und dem Nachmittag herstellen: polyzentrische Elemente der Kooperation, der Koordination und vielleicht dann sogar der Legitimation. Es geht also um einen grundsätzlichen wissenschaftlichen Brückenschlag. Er müsste uns doch heute schon gelingen; spätestens aber die nächste Generation kann in dreißig Jahren sogar beide Tage in Rostock insgesamt miteinander verbinden. Fritz Werner hat nun einmal einen lebendigen Klassikertext geschaffen, den wir „europäisieren“ sollten: europäisches Verwaltungsrecht als „konkretisiertes Europäisches Verfassungsrecht“".

Diskussionsleiter: Danke, Herr Häberle, für einen Einwurf, der normalerweise von beißender Schärfe sein müsste; Ihnen ist es stattdessen sogar gelungen, eine brückenbauende Anmerkung zu machen.

Rengeling: Auch ich möchte noch einmal betonen, dass bei dem Dickicht des Stoffes, der zu bewältigen war, zwei sehr schöne „Schneisen“ durch den Bereich des Verwaltungsverbundes mit all seinen Verästelungen gezogen worden sind. Wir haben aber am Ende dieser Schneisen sicher auch den Eindruck, dass es richtig ist, was Herr Paul Kirchhof gesagt hat, dass nämlich immer noch viele Unklarheiten im Hinblick auf die Verantwortung und insbesondere auch auf die Legitimation bestehen. $\mathrm{Zu}$ dem letzten Punkt möchte ich etwas sagen, das im Ansatz abweicht von dem, was Herr Hoffmann-Riem vorgetragen hat. Sicher ist es gut, grundsätzlich ein weites Verständnis von Legitimationsbausteinen zu haben. Aber ich frage mich doch: Kann man tatsächlich demokratische Legitimation herstellen durch Beteiligung, wie Sie es ungefähr 
gesagt haben, Herr Pache? Ist tatsächlich die Beteiligung am Verwaltungsverfahren ein demokratischer Legitimationsbaustein? Wenn das so ist, dann möchte ich eine weiterführende Frage stellen, und zwar: Muss man nicht zwischen der Legitimation von Gesetzgebung und Verwaltung unterscheiden? In einer These von Herrn Groß werden die abstrakt-generellen Normkonkretisierungen erwähnt. Diese können in unterschiedlichen Bereichen vorgenommen werden. Sie können innerhalb der Verwaltung erfolgen, sie können aber auch innerhalb der Gesetzgebung erfolgen. Auf der EG-Ebene ist es z.B. üblich, in Anhängen zu einer Richtlinie den Stand der Technik festzuschreiben. Das ist Gesetzgebung. Wie ist es dann mit der demokratischen Legitimation durch Beteiligung? Die Beteiligung bei dieser Gesetzgebung ist sicher reduziert im Vergleich zu der Normkonkretisierung im Bereich der Verwaltung. Und wie ist in diesem Zusammenhang die Frage zu sehen, die auch schon angeschnitten worden ist, nämlich von Herrn Hermes: Bietet hier die sogenannte Fachverwaltung einen Ansatz? Ist Beteiligung ein Legitimationsbaustein im Sinne einer demokratischen Anforderung der Rechtsetzung oder der Verwaltung etwa auf EG-Ebene?

Lepsius: Die Referenten haben monistische Legitimationsstrukturen verabschiedet. Herr Hermes hat das wortmächtig befürwortet. Aber entspricht die Vorstellung monistischer Legitimationsstrukturen eigentlich der Realität? Stellen wir uns die einfache Frage: Das bayerische Landratsamt erlässt eine Baugenehmigung. Wie wird sie demokratisch legitimiert? Der Bundestag verschafft sachlich-inhaltliche demokratische Legitimation indem er das BauGB erlässt. Wenn der Bundesrat dem BauGB zustimmt, werden die Legitimationsstrukturen schon ziemlich schwierig. Hinzu tritt die sachlich-inhaltliche Legitimation der Baugenehmigung durch die Landesbauordnung, die über den Bayerischen Landtag auf ein anderes Legitimationssubjekt gestützt wird. Hinzu tritt schließlich eine kommunale Bauleitplanung und damit ein weiteres Legitimationssubjekt. Im Hinblick auf die personelle Legitimation der Baugenehmigung ist der in Bayern direkt gewählte Landrat zu nennen, daneben das Landratsamt mit seinen Staatsbediensteten. Verzeihung Herr Hermes, aber in diesem Legitimationsgeflecht erkenne ich keine monistischen Legitimationsstrukturen. Wir brauchen sie daher nicht zu verabschieden, weil es sie nicht gegeben hat. Dieses, denke ich, sollte klargestellt werden. Beide Referenten haben sich in Abkehr von den herkömmlichen Legitimationsstrukturen jedoch für eine „Output-Legitimation" stark gemacht. Dabei handelt es sich um ein Theorieangebot aus der Politikwissenschaft, das mich immer sehr interessiert hat. Als ich die politikwissenschaftliche Literatur las, beschlich mich die Vermu- 
tung, bei der „Output-Legitimation“ fallen Legitimationssubjekt und Legitimationsobjekt zusammen. Dann aber verschaffte die „Output-Legitimation“ nur ihrem Namen nach Legitimation. Als Juristen können wir von Legitimation indes nur sprechen, wenn Subjekt und Objekt getrennt sind. Es muss ein Subjekt benennbar sein, von dem die Legitimation ausgeht. Nachdem sich jetzt Juristen der „Output-Legitimation“ bedienen, muss ich davon ausgehen, dass es ein vom Objekt unterscheidbares Legitimationssubjekt bei der „Output-Legitimation“ tatsächlich gibt. Ich erhoffe mir also Antwort auf eine durch die Lektüre der politikwissenschaftlichen Literatur noch nicht gelöste Frage, nämlich Antwort auf die Frage: Wer ist eigentlich das Legitimationssubjekt bei der „Output-Legitimation"?

Gröschner: Als bekennender Alteuropäer hatte ich heute vormittag ein prinzipielles Problem mit dem europarechtlichen Neoliberalismus, der beide Berichte durchzog. Insofern ließ mich Leitsatz 1 des nachmittäglichen Referats hoffen. Denn dort war ausdrücklich die Rede von der Konkretisierung und Verwirklichung des „Gemeinwohls“, also in alteuropäischer Tradition einer genuin republikanischen Idee. Dann musste ich jedoch in Leitsatz 18 lesen, „dass das Gemeinwohl Teil des demokratisch geforderten Verwaltungshandelns auch insofern ist, als es um die bestmögliche und effektive Erledigung der übertragenen Aufgaben“ gehe. Sollte es wirklich wahr sein, dass im gegenwärtigen Diskurs des Europarechts das ciceronische Motto „salus populi suprema lex“ entweder in Vergessenheit geraten ist oder demokratisch im heutigen Sinne verstanden wird, obwohl es das Volk in Rom nie zum Träger von Herrschaftsgewalt gebracht hat? Ciceros höchstes Gesetz war die republikanische Herrschaft für das Volk, nicht die demokratische Herrschaft durch das Volk. Ich bin überzeugter Europäer, aber nicht um den Preis der Preisgabe eines Legitimationsmodells, in dem die Konkretisierung des Gemeinwohls Aufgabe der Amtswalter einer Republik ist. In diesem Modell kommt es darauf an, dass im Amt - etwa eines bayerischen Landrats und seiner Bediensteten - mit Urteilskraft und Witz agiert wird. Wir wollen keine witzigen Bürokraten, sondern gewitzte. Das ergäbe dann sogar eine eigene republikanische Methodenlehre. Niemand bestreitet die Notwendigkeit demokratischer Legitimationsketten. In Fragen des Gemeinwohls geht es aber um eine republikanische Legitimationskette, die von der Gemeinwohlidee über öffentliche Interessen, Aufgaben und Befugnisse bis zur Entscheidung im konkreten Einzelfall reicht. 
Biehler: Es bietet sich einfach an, an das römische Staatssymbol SPQR, senatus popolusque romanorum, zu denken. Auch das Volk, populus, war natürlich Legitimationsträger im klassischen Rom. Also, Herr Gröschner, da müssen wir vielleicht noch mal zusammenkommen, was wir sowieso tun sollten.

Ich möchte eine weitere Begrifflichkeit in das Spiel bringen. Wir haben viel von Legitimation und Verantwortlichkeit gesprochen. Da ist ein sehr sehr enger Zusammenhang zu sehen, sogar bis ins Rechtliche hinein. Herr Groß Sie haben von der untauglichen Konzeption der EU Mehrebenenverwaltung gesprochen, die nicht auf Demokratieunfähigkeit der Mehrebene zurückzuführen ist und ich glaube da ist auch ein ganz wichtiger Punkt zu sehen. Die Verantwortlichkeit in dieser Mehrebenenverwaltung ist ein bisher nicht gelöstes und nicht wirklich erfasstes Problem. Herr Pernice hat dankenswerter Weise das mal auf ganz konkrete Punkte zurückgeführt. Telekommunikation, die Ministerweisung, die eben in diesen Kooperationsentscheidungen nicht mehr wirklich greifen kann. Deswegen wollte ich einmal auf den Art. 288 des EGVertrages hinweisen. Die Amtshaftung kann sozusagen ein Litmustest oder ein Gegentest für Verantwortlichkeit vielleicht sein und damit eine neue Erkenntnismöglichkeit wie hier die Verantwortungsstruktur in Mehrebenensystemen wirklich einzuordnen ist. Bei der Untersuchung dieser Verantwortlichkeit im Sinne der Amtshaftung, die im nationalen Bereich uns ja sehr vertraut ist, muss man eben feststellen, dass diese in der Mehrebenenverwaltung bei weitem noch nicht den nationalen Standard erreicht hat. Es besteht insbesondere eine Tendenz, auf europäischer Ebene (Teil-)Verantwortung nicht wahrzunehmen. Ich erinnere besonders an den Fall Dorsch, den ich in diesem Zusammenhang der drei Ebenen Völkerrecht, Europarecht, nationales Recht immer noch für symptomatisch halte. Es wäre ein neuer Ansatz oder Forschungspunkt, hier über Verantwortung und Mehrebenenlegitimation nachzudenken.

Es ist eine große Versuchung und ich werde hier nicht ganz widerstehen, Herr Kirchhof, Sie haben Herrn Häberle als Brückenbauer bezeichnet. Ich will das mal ins Lateinische übersetzen. Der Pontifex, der hier natürlich, wenn wir von großen und kleinen Kollegen sprechen, der Titel Pontifex maximus ist ja nun schon an einen emeritierten Kollegen der theologischen Fakultät Tübingens vergeben, aber ich wollte hier deshalb den noch vakanten Pontifex minimus einmal vorschlagen. Der große und der kleine Brückenbauer. Und damit will ich es dann auch belassen.

Diskussionsleiter: Ich danke ihnen auch im Interesse von Herrn Pieroths Sekretärin, dass sie von den altgriechischen Redewendungen jetzt auf die leichter verständlichen lateinischen Versionen übergegangen sind. 
Meine Damen und Herren, jetzt muss ich Sie um eine Genehmigung bitten zu einer Abweichung von unserer Regel. Sie wissen, bei uns gilt das eherne Gesetz der Ampel. Ich würde den nächsten Redner gerne ohne Ampel reden lassen. Er hat geschrieben: Ich bitte um eine Minute extra. Aber ich glaube er wird etwas länger brauchen. Es spricht jetzt Herr Raschauer. Die Begründung, dass wir die Ampel nicht anschalten, ist zweifach. Erstens redet Herr Raschauer in der Zwischenzone des Themas „Legitimation“ zu dem Tagesordnungspunkt 3 unserer Diskussion; das wären schon zweimal drei Minuten, also er hätte eigentlich schon sechs Minuten Redezeit. Zweitens will er uns, weil unter den Referenten kein Österreicher ist, einige österreichische Besonderheiten und Aktualitäten nahe bringen. Also sehen Sie mir nach, wenn ich die Ampel nicht anschalte. Ich weiß, dass ich damit eine offene Flanke für etwaige helvetische, übertemporäre Beiträge biete, aber das nehme ich auf meine eigene Kappe.

Raschauer: Danke für die freundliche Einleitung. Ich werde die Zeit gewiss nicht überstrapazieren. Einige Punkte scheinen mir erhellungsbedürftig. Die Strategie des Referats von Herrn Groß war in gewisser Weise dadurch charakterisiert, dass er uns damit „erschlagen“ hat, dass es doch schon viele Fälle von Kooperation und Koordination gibt, die er generell-abstrakt angesprochen hat. Da drängen sich zwei Probleme auf. Es gibt tatsächlich viele Arten von Agenturen. Es gibt tatsächlich viele Arten von Ausschüssen im Gemeinschaftsrecht. Aber im Hinblick auf das Thema wären doch bestimmte Einrichtungen herauszuarbeiten gewesen, die selbständige Entscheidungsbefugnisse haben. Denken wir an die Agentur in Sevilla, die BAT-Dokumente erarbeitet. Sie bildet kein rechtsstaatliches Problem und kein demokratietheoretisches Problem. Aber es gibt sensible Agenturen und es gibt sehr sensible Ausschüsse, die eigenständige Entscheidungsbefugnisse haben und nicht mit einfachen Modellen der Komitologie erfassbar sind. Zweitens - und das zum österreichischen Recht - sind die Verbandskompetenzen in diesem Rahmen ein wenig durcheinander geraten. Die österreichischen Beispiele sonst will ich lieber nicht dazu Stellung nehmen - waren durchwegs aus dem Bereich der mittelbaren Bundesverwaltung gewählt. Das heißt, Bundesaufgaben werden von Landesorganen als Bundesverwaltung geführt. Es geht um Bundesaufgaben, Bundesgeld und Bundeshaftung. Soll das der Bund nicht steuern? Ich sehe das Problem nicht. Das ist eine ähnliche Konstruktion wie die Organleihe. Aus diesem Grund habe ich auch kein Problem mit dem Rechnungsabschlussverfahren im EGAgrar-Marktordnungsrecht; da gibt es problematischere Bereiche. Und daher - in Überleitung zum Referat von Herrn Pache - der Hinweis auf 
eine Aktualität in Österreich. In diesen Tagen ist das Verhältnis zur Europäischen Union Gegenstand eines Verfahrens vor dem Verfassungsgerichtshof. Er prüft das Emissionszertifikategesetz - in Deutschland ist die Materie im TEHG, im Zuteilungsgesetz und im PrMechG geregelt, bei uns in einem Gesetz. In diesem Gesetz findet sich der Satz, dass der nationale Zuteilungsplan nach Maßgabe von Änderungen der europäischen Kommission kundzumachen ist. Der „Fehler“, den der Gesetzgeber gemacht hat, besteht darin, dass er die Entscheidung der Kommission überhaupt erwähnt hat; nach der Richtlinie ist sie ohnedies erforderlich. Deshalb geht es nun um etwas einigermaßen Sensationelles, der Verfassungsgerichtshof prüft die Verfassungsmäßigkeit des $\mathrm{Zu}$ stimmungserfordernisses der Kommission. Insider rechnen mit einer Aufhebung. Es wurde vorhin so treffend formuliert: Es gibt im Grundgesetz vorgezeichnete Vollzugsmodelle. Die gibt es auch im B-VG, und da kommt die Europäische Kommission nicht vor (Anmerkung: Mittlerweile wurde die gesetzliche Bestimmung mit Erkenntnis vom 11. 10. 2006 tatsächlich mit dieser Begründung aufgehoben). Das hat nun Auswirkungen auf andere Bestimmungen - ich sehe Herrn Pernice an: Nach meiner Erinnerung ist nicht nur die nationale Verordnung über die Marktabgrenzungen nach Telekomrecht der Kommission zur Zustimmung vorlegen, die ein Widerspruchsrecht hat. Wie ist der Akt dann zu sehen? Wessen Akt ist das? Ist das ein der EG zurechenbarer Akt oder ein Österreich zurechenbarer Akt? Oder etwa die bei uns sogenannte Lex Nabucco. Meines Wissens haben auch Sie eine solche große Gaspipeline. Die Nabucco-Pipeline wird eine Ausnahme vom Netzzugang nach nationalem Recht erhalten; dafür ist aber wiederum die Zustimmung der Kommission erforderlich. Wessen Entscheidung ist die Freistellung vom Netzzugang dann? Man kann wohl nicht von einer autonomen Entscheidung einer österreichischen Behörde sprechen. Ich bin Ihnen sehr dankbar, Herr Pache, dass Sie das in Ihrer These 22 angesprochen haben. Man wird wahrscheinlich auf europäischer Ebene einen Ausweg suchen müssen, indem man etwa eine Typologie von Kooperationsformen oder dergleichen vorzeichnet. Denn wenn der österreichische Verfassungsgerichtshof die erwähnte Bestimmung aufhebt, brauchen wir möglicher Weise eine Verfassungsbestimmung. Aber was soll wir in diese hineinschreiben? „Was immer die Kommission will, ist rechtserheblich“ - so wird es wohl nicht gehen. Zuletzt noch ein Punkt, der etwas unterbelichtet blieb in den Referaten: Was als einigermaßen unangenehm hinzu tritt bei der heute geläufigen Art von Kooperation, ist das subnormative Recht der Europäischen Kommission. Ich bin Mitautor des Entwurfs des österreichischen Umwelthaftungsgesetzes in Deutschland heißt es Umweltschadensgesetz und liegt ebenfalls als 
Ministerialentwurf vor. Es geht um eine Richtlinie, die wir beide umzusetzen haben. Meinen Vorstellungen wurden von den Beamten des Ministeriums entgegen gehalten, dass sie jetzt schon bei drei Besprechungsrunden mit dem Leiter der Rechtsabteilung der zuständigen Generaldirektion waren, und dieser habe gesagt die Richtlinie sei so und so zu interpretieren. Sie ahnen was das im Beamtenbereich bedeutet. Da stoße ich mit meiner Beratungskompetenz an eine Mauer. Oder im Energierecht, bei der Umsetzung des unbundling nach den Richtlinien. Kein Mensch schaut noch in die Richtlinien, jeder zieht nur die so genannten Leitlinien der Kommission heran. Übrigens zum Thema Publizität: Man muss schon gut sein, um diese Leitlinien im Internet zu finden. Aber was für eine Art von „Rechtsquelle“ ist das? Oder unser Ökostromgesetz - bei Ihnen heißt es Gesetz über Erneuerbare Energien. Wir haben es konzipiert nach der PreußenElektra-Entscheidung des EuGH, die Kommission hat dennoch ein Jahr lang gehämmert, dass das eine Beihilfe sei. Nach einem Jahr sind die Politiker schwach geworden. Das ist eine Art von Einflussnahme, die in den Referaten überhaupt nicht angesprochen worden ist, die aber permanenter Alltag in allen Vollzugsbereichen ist. Daher bin ich begeistert vom Votum von Herrn Kirchhof: Diese Entwicklungen muss man problematisieren und nicht nur zur Kenntnis nehmen. Danke für ihre Geduld.

Streinz: Ich habe einige Fragen zu praktischen Problemen der Verantwortung und Verantwortlichkeit. Die erste betrifft nicht die Effizienz, sondern die Effektivität des Vollzugs des Gemeinschaftsrechts im Mehrebenensystem. Heute Vormittag ist von der Bindung der Rechtsetzung durch den Vorrang des Gemeinschaftsrechts gesprochen worden. Aber der Anwendungsvorrang des Gemeinschaftsrechts, seit 40 Jahren gefestigte Rechtsprechung, betrifft ja nicht nur die Gesetzgebung, sondern alle Staatsgewalten und somit auch die Judikative und die Exekutive. Bei der Judikative erscheint der Anwendungsvorrang ziemlich real zu sein. So hat der Bundesgerichtshof das alte Verbraucherleitbild, das in Deutschland herrschte, nämlich das des an der Grenze der Debilität vorhandenen Verbrauchers, durch das Leitbild des verständigen Verbrauchers ersetzt und, auch das Oberlandesgericht Köln ist dem jetzt mittlerweile gefolgt. Ich habe eben gelesen, dass jemand, der in eine Tafel Nussschokolade beißt, damit rechnen muss, dass da auch eine harte Nuss drin sein kann. Auch im Strafrecht gibt es Beispiele. Aber wie steht es eigentlich mit dem Verwaltungsvollzug? Wie ist hier die Realität des Binnenmarkts? Gibt es die Durchsetzung der Grundfreiheiten des Bürgers, von denen heute Vormittag die Rede war, durch die Verwaltung? Das Problem der Verwaltung ist ja, dass sie nicht den Euro- 
päischen Gerichtshof im Vorentscheidungsverfahren anrufen können. Daher meine Frage: Gibt es außer aktuellen und strittigen Fällen hinsichtlich des deutschen Apothekenrechts und des deutschen Lotterierechts Anwendungsfälle oder steht hier der Anwendungsvorrang nur in den Lehrbüchern? Die zweite Frage: Es wurde von Unschärfen in der Verantwortlichkeit in der Mehrebenenverwaltung gesprochen. Dazu ein Beispiel: Es gibt im Lebensmittelrecht ein Schnellwarnsystem auch auf europäischer Ebene. Das Schnellwarnsystem funktioniert ja auch zum Teil auf nationaler Ebene nicht. Wenn aus Mannheim gemahnt wird, wird in München nicht immer richtig kontrolliert. Zur europäischen Ebene folgender Fall: Aus einem Mitgliedstaat erfolgte eine Warnung, dass ein Verstoß gegen das Lebensmittelrecht vorliege, der auch gesundheitsgefährlich sei. Diese geht an die Kommission, die Kommission meldet es weiter. Im anderen Mitgliedstaat wurden daraufhin Produkte beanstandet und beschlagnahmt. Hinterher stellt sich heraus, dass die Warnung unbegründet war. Frage: Wer ist jetzt hier verantwortlich und wer haftet? Der Betroffene wandte sich an die Kommission. Die Kommission sagte, und vom Gericht erster Instanz, dem EuG, wurde dies bestätigt: „Wir sind nur die Drehscheibe. Wir geben das einfach weiter, wir haften für nichts“. Derjenige Mitgliedstaat, der die Maßnahme durchführte, verwies auf die Mitteilung der Kommission. Was Besseres konnte ihm gar nicht passieren, als sich auf die Autorität der Kommission zu verlassen. Kann der Betroffene sich jetzt an den Erstmelder wenden und was wird dieser einwenden? Ein letzter Punkt: Das Problem der Verantwortlichkeit und der Zuweisung von Verantwortlichkeit. Herr Häberle hat gesagt, dass man die Betreffenden zur Verantwortung ziehen können muss, auch bei den Europawahlen etc. Aber dafür muss man wissen, wer für was eben verantwortlich gewesen ist. Und diese Verantwortlichkeit und Haftung führt auch zu einer Verkomplizierung im Verwaltungssystem. Ich war eben auf einer Konferenz bei der Kommission in Brüssel, bei der ging es um die Verbesserung, Vereinfachung der Rechtsetzung im Agrarbereich. Unter anderem wurde das Problem von Mehrfachkontrollen aufgeworfen. Es kann einem Landwirt passieren, dass bis zu fünf Kontrolleure nach einander zu ihm kommen, und jeder beruft sich darauf, dass er dafür hafte, dass das, was er vornehme, richtig ist, er somit selbst kontrollieren müsse. Als nächstes kommt der Europäische Rechnungshof, der sich auf gar niemand anderen verlassen könne und daher auf alle Fälle kontrollieren müsse. Daher die Frage: Wie kann man solche im Mehrebenensystem und in der Mehrfachverwaltung auftretenden Doppelungen und Verdreifachungen etc. reduzieren? 
Hufen: Herr Streinz hat schon ein wenig die harte Ebene der Tatsachen des Europäischen Verwaltungsrechts im Lebensmittelrecht angesprochen. Da kann ich anschließen. Mein Thema ist der Aspekt „Europäisches Gemeinwohl" in den Thesen 1 und 4 von Herrn Pache. Es ist schon interessant: Eine Staatsrechtslehrertagung befasst sich mit dem Thema „Verwaltung“ und im Mittelpunkt stehen nicht die Ergebnisse des Verwaltungshandelns sondern im Grunde genommen Relationsbegriffe wie Verantwortung, Effizienz usw. Da fragt man sich natürlich: Verantwortung wofür? Effizienz im Bezug worauf? Bietet da ein europäisches Gemeinwohl möglicherweise einen Bezugspunkt? Ich fürchte nein. Wir sind zwar alle an den Gedanken der gemeinwohlorientierten einen richtigen Entscheidung gewöhnt, aber im Europarecht scheint mir das noch mehr eine Fiktion zu sein als im nationalen Verwaltungsrecht. Gemeinwohl verwirklicht sich hier allenfalls im Verfahren und relational. Im europäischen Bereich gibt es sogar mindestens drei Problemebenen, die der Definition eines einzig richtigen gemeinwohlorientierten Ergebnisses entgegenstehen: Da sind auf der vertikalen Ebene die nationalen Interessen. Selbst wenn wir das Gemeinwohl etwas weniger idealistisch und abstrakt formulieren - etwa im Sinne des Vorsorgeprinzips, des Verbraucherschutzes, des Klimaschutzes usw., dann wird eben deutlich, dass ein französischer Winzer, ein dänischer Krabbenfischer, ein polnischer Schweinezüchter darunter etwas ganz Unterschiedliches verstehen - von einem Transportunternehmen aus Portugal, das deutsche Autobahnen am Sonntag befahren will, ganz abgesehen. Die Gegensätze gehen bis zum Sitz europäischer Behörden. Auch hier geht es nicht um Gemeinwohl oder Optimierung, sondern um schlichte nationale Interessen und Proporz. So konnte gerade mit Mühe verhindert werden, dass die Europäische Lebensmittelbehörde in Finnland bzw. in Irland angesiedelt wird, wo bekannterweise am wenigsten Lebensmittel produziert werden. Jetzt ist der Sitz Parma, da gibt es wenigstens den bekannten Schinken. Interessengegensätze gibt es aber auch in horizontaler Ebene. Hier müssen wir zur Kenntnis nehmen, dass die europäischen Interessenverbände wohlorganisiert sind, dass sie mit den versäulten Fachbruderschaften auf europäischer Ebene hoch effizient zusammenarbeiten und dass dort zumindest teilweise definiert wird, was europäisches Gemeinwohl ist. Ein dritter Punkt ist die Zeitebene: Europäisches Verwaltungsrecht ist zeitlich nicht ungebunden wie das „traditionelle Gemeinwohl“, das Herr Gröschner ins Spiel gebracht hat. Europäisches Verwaltungsrecht ist leider in hohem Maße tages- - um nicht zu sagen skandalorientiert. Gerade im Lebensmittelrecht wird es ganz deutlich. Man reagiert und läuft hinter der jeweils nächsten Krise (BSE-Krise, Hühnerpest-Krise usw.) hinterher. Fazit: Die Hoffnung auf ein vorgege- 
benes Gemeinwohl, wenn es jemals im nationalen Verwaltungsrecht etwas solches gab, trügt im Europarecht gewaltig. Daraus wird deutlich, dass es eigentlich nur verfahrensmäßig, sozusagen induktiv gehen kann und hier sind auch die Schlussfolgerungen beider Referenten eigentlich ganz klar: Wir müssen setzen auf transparente Verfahren, wir müssen setzen auf Begründungsstrukturen und auf die Einführung einer europäischen Verwaltungsgerichtsbarkeit und dem Bürger wirklich den Eindruck geben, dass seine Rechte in den Entscheidungsvorgängen aufgehoben sind. Letztlich sind es dann vielleicht doch die Dezentralität, Subsidiarität und die Bürgernähe, die eine bessere Antwort auf die Fragen geben, die wir uns heute stellen.

Gröschner: Herr Hufen gibt mir das Mikrofon sozusagen als Staffelstab weiter. Das Gemeinwohl ist natürlich nicht vorgegeben, sondern aufgegeben, meinetwegen als regulative Idee im kantischen Sinne. Und wenn die Brüsseler Bürokratie sich ausgerechnet in Parma um Lebensmittelsicherheit kümmert, dann nehme ich dies als schöne Gemeinsamkeit zwischen einem Parmaschinken und dem Gemeinwohl - nicht im ganzen, sondern scheibchenweise zu genießen.

E. Hofmann: Herr Groß, Herr Pache, Sie haben ja beide, fand ich, in sehr großer Anschaulichkeit die hohe Komplexität der rechtlichen Regularien bezüglich von Entscheidungsvorgängen in der Mehrebenenverwaltung aufgezeigt. Die Normen, die das regulieren, habe ich nicht vermisst, wie Herr Jestaedt. Ich denke an das Europarecht, wo es eine Unmenge von Regularien gibt, wie die verschiedenen Ebenen miteinander kooperieren sollen. Das Planungsrecht lehrt aber, dass es da ein gewisses Problem mit informellen Verfahren gibt. Je komplexer das Zusammenspiel der Akteure ist, desto größer ist die Gefahr der Verantwortungsvermeidung durch die Flucht ins informelle Verfahren, dass also die Flurgespräche darüber entscheiden, was passiert, und nicht so sehr das formelle Verfahren. Ich frage mich, ob diese Entwertung der formellen Verfahren in den Mehrebenenverwaltungen, die Sie besprochen haben, nicht auch eine große Gefahr darstellen und wenn ja, ob es nicht irgendwelche Mechanismen gibt, um dieser Flucht aus den formellen Verfahren zu begegnen. Es scheint da ein gewisses Paradox zu geben. Je strenger wir sind mit den Regularien, desto größer ist der Druck auf die Flucht, also die Flucht ins informelle Verfahren, und je „lockerer" wird sind, desto intransparenter wird es.

Breuer: Vorwegschicken möchte ich, dass ich zu denjenigen gehöre, die die klassischen Prinzipien der demokratischen Verantwortung und der 
rechtsstaatlichen Transparenz und Strukturen noch nicht ganz aufgegeben haben. Ein spezieller Punkt, der mir aufgefallen ist, besteht darin, dass man auf der europäischen Ebene nun Leitlinien oder, englisch formuliert, guidelines bevorzugt, um unklare oder unvollständige Normen zu konkretisieren und sie dann anwendungsfähig zu machen. Hier fällt natürlich dem deutschen Staats- und Verwaltungsrechtler unvermeidlicher Weise die Parallele zu den Verwaltungsvorschriften ein. Bekanntlich haben wir uns darüber belehren lassen müssen, dass die Verwaltungsvorschriften keine ordentliche Rechtsform seien, da sie weder demokratischen noch rechtsstaatlichen Anforderungen entsprächen. Wenn man dies aber einmal zu Grunde legt, vermag mir nicht einzuleuchten, weshalb nun die Kommission auf der Europäischen Ebene mit solchen Leitlinien oder guidelines operieren kann. Die guidelines gehören nicht zu den Rechtsformen des Gemeinschaftsrechts nach Art. 249 EGV, sie sind ein informelles Instrument. Entweder muss es gelingen, auf der europäischen Ebene ordentliche Rechtsnormen in den Handlungsformen des Art. 249 EGV zu erlassen, dann ist die europäische Kompetenz einwandfrei ausgeübt, und dann greifen auch die demokratischen und die rechtsstaatlichen Prinzipien auf der europäischen Ebene. Oder es gelingt nicht, auf der europäischen Ebene einen ordentlichen Rechtsakt zu erlassen, dann kann man aber auch nicht ersatzweise einen informellen Rechtsakt erlassen. Ich würde also sehr davor warnen, das Instrument der Leitlinien oder guidelines hier zum Zukunftsprinzip zu erheben. Im Gegenteil; wenn für die nationale Ebene solche Instrumente nicht zugelassen sind, dann können sie auch auf der europäischen Ebene nicht zugelassen sein. Und wenn wir so verfahren, dann kann man wieder mit der Einhaltung demokratischer und rechtsstaatlicher Strukturen auf allen Ebenen zufrieden sein. Im Augenblick bin ich aber noch beunruhigt - es sei denn, die Referenten wären in der Lage, die Sorgen zu beseitigen, die jedenfalls bei mir aufgekommen sind.

Schmidt-Aßmann: Ich möchte auf eine Funktionsbedingung aufmerksam machen, die hinter der Dogmatik der Europäischen Verwaltungskooperation liegt, nicht selbst einen Begriff der Verwaltungsrechtsdogmatik, sondern eine Voraussetzung wirksamer Verwaltung im Unionsraum darstellt: das interadministrative Vertrauen. Ohne ein solches Vertrauen lassen sich wirksame Informationsbeziehungen zwischen den beteiligten Verwaltungen - vertikal zwischen den Verwaltungsinstanzen der Europäischen Union und den Mitgliedstaaten sowie horizontal zwischen den Mitgliedstaaten - auf Dauer nicht gewährleisten. Besonders deutlich wird das an Entscheidungen einer einzelnen nationalen Behörde, die nach dem Anerkennungsprinzip transnationale gemeinschaftsweite Wirkungen entfal- 
tet. Hierzu ist nicht nur eine Harmonisierung des anzuwendenden Rechts, sondern vor allem die Gewährleistung vergleichbarer Qualitätsstandards in den Verwaltungen notwendig: Kompetenz, Loyalität, Neutralität und Unbestechlichkeit. Vertrauen bildet sich durch Erfahrung. Es muss eine Erfahrungstatsache sein, dass die am Verwaltungsverbund beteiligten Verwaltungen diesen Anforderungen entsprechen. Fehlt es daran, kommen die notwendigen Kooperationsbeziehungen nicht zustande oder bleiben oberflächlich. In einem bundesstaatlichen Verwaltungsgefüge mit langer Tradition hat sich dieses Vertrauen zwischen Verwaltungen des Bundes und der Länder längst herausgebildet und wird im Regelfall nicht als ein besonderes Problem wahrgenommen. Für die Europäische Verwaltung, die sich erst nach und nach konstituiert und Länder mit sehr unterschiedlichen Verwaltungstraditionen und Qualitätsstandards umfasst, muss das zu einem eigenen Thema gemacht werden. Es erscheint unsicher, dass die Funktionäre der EU in der Euphorie mancher Beitrittsverhandlungen hierauf geachtet haben. Sollte das nicht der Fall sein, wäre das ein gefährlicher Fehler. Der Hinweis auf bestimmte Aufsichtsmittel der EG-Kommission reicht nicht aus. Das notwendige interadministrative Vertrauen muss durch strukturelle Maßnahmen weit im Vorfeld konkreter Kontroll- und Aufsichtsvorgänge aufgebaut werden. In beiden Referaten des heutigen Nachmittags sind wichtige rechtsdogmatische Scharniere des Europäischen Verwaltungsverbundes dargestellt worden. Ich möchte anregen, diese Dogmen noch einmal daraufhin durchzusehen, inwieweit in ihnen auch der Gesichtspunkt des Vertrauens mitverarbeitet worden ist. Wir brauchen eine vertrauensgenerierende Dogmatik, die mit einem Blick für Zusammenhänge, d.h. systematisch, zu entwickeln ist. Um ein solches Denken in die europäische Verwaltungsrechtswissenschaft einzubringen, ist gerade das deutsche Verwaltungsrecht gut gerüstet. Man sollte sich hier nicht zu schnell in Selbstbeschränkung üben und auf den zufälligen, politischen und eben unsystematischen Charakter der Rechtsetzung in der EG verweisen. Es gibt gerade in jüngerer Zeit Beispiele, dass die Vorzüge eines systematischen Rechtszugangs auch in der europäischen Rechtsetzung durchaus gesehen werden. Ein erster Schritt ist schon die Kodifikation von Rechtsakten, wesentlich weiter geht etwa die organisationsrechtliche Modellbildung, die in der Verordnung über die Regulierungsagenturen sichtbar wird. Insgesamt hat die Rostocker Tagung ein Qualitätsmerkmal auf jeden Fall verdient: Wir haben heute ein erstes Mal wirklich zentral über die Europäische Verwaltung gesprochen, während unser Vorstellungsbild bei früheren Tagungen (aus gutem Grund) zunächst die nationale Verwaltung war. Künftig wird man bei verwaltungsrechtlichen Themen stets die nationale und daneben die supranationale 
und die transnationale Schicht mitbedenken müssen. Das ist heute in Referaten und Diskussionen klar geworden und kann als ein Meilenstein gelten.

Masing: Eine Bemerkung, die anschließt an den Beitrag von Herrn Lepsius: Ich glaube in der Tat, der Kampf gegen die Lehre eines demokratischen Monismus erübrigt sich, wenn man unter diesem Kampf die Durchsetzung der Einsicht versteht, dass es nicht nur das Bundesvolk, sondern auch das Landesvolk und auch kommunale Körperschaften gibt, die miteinander verschachtelt Legitimation spenden. Das ist in der Tat trivial. Aber es ging in den Referaten, insbesondere in dem Referat von Herrn Groß, natürlich um mehr. Es ging um polyzentrische Legitimationssubjekte, die wechselnd sind, die erst durch die einfache Rechtsordnung geschaffen und konstituiert werden, d.h. um Legitimationssubjekte, die nicht zu den festen kollektiven Grundlagen gehören, welche auf Verfassungsebene anerkannt sind. Und ebenso ging es um Akteure, die zu diesen in gewisser Distanz stehen. Auch da, denke ich, ist aber der Zugriff des Referats richtig: Wir kommen in der modernen Welt nicht umhin, Verwaltung auf viele Legitimationsstufen zu verteilen und diese damit auf verschiedenen Ebenen zu strukturieren. Zentral ist dabei allerdings, dass eine solche polyzentrische Gestaltung wirksam an parlamentarische Entscheidungen rückgekoppelt bleiben muss, etwa durch Berichtspflichten, durch strenge Rechtfertigungspflichten, durch Transparenzpflichten. Um ein Beispiel aus dem Telekommunikationsrecht oder überhaupt aus dem Regulierungsrecht zu nennen, sei hier nur auf die Berichtspflichten, die etwa der Regulierungsbehörde selbst obliegen, verwiesen oder auf die der Monopolkommission, die eine Außenbeobachtung installieren. Diese Berichte zielen auf eine regelmäßige parlamentarische wie gouvernementale Befassung mit der Arbeit der Behörde. Eine polyzentrische Ordnung muss also ihrerseits rückgekoppelt und gestaltet sein. Damit bekommt die These von der Ausgestaltungsfähigkeit und - bedürftigkeit der Legitimation einen Gehalt, der trotz der Anerkennung verschiedener Ebenen im Kern doch auf eine parlamentarisch zentrierte Verantwortung zurückführt. Insoweit scheint mir denn auch die Rede von der „outputorientierten“ Legitimation irreführend: Nicht ein gutes „output" legitimiert, sondern die parlamentarische Entscheidung und Verantwortung. Auch dieses Modell stößt allerdings an Grenzen - oder so scheint es zumindest -, wenn man etwa den Fall der internationalen Abstimmung zwischen den nationalen Regulierungsbehörden im Bereich der Telekommunikation in den Blick nimmt. Freilich ist diesbezüglich davor zu warnen, die Anforderungen des Demokratieprinzips von den Grundvorstellungen her zu überziehen. Bei 
manchen wird aus der verfassungsrechtlichen Forderung ,alle Staatsgewalt geht vom Volke aus" die Vorstellung, die gesamte gesellschaftliche Entwicklung geht von Entscheidungen des Volkes aus. Das aber meinte das Grundgesetz nie und kann auch nicht gemeint sein. Auch wenn die Politik, etwa in Verhandlungen - welche immer im Wege des gegenseitigen Nachgebens unter gegebenen Kräfteverhältnissen stattfinden -, natürlich nicht frei ist in der Durchsetzung eigener Vorstellungen, dann widerspricht das dem Demokratieprinzip nicht. Wenn wir 1955 mit den Alliierten um die Souveränität verhandelt haben, so haben wir die Entscheidung doch nicht deshalb für undemokratisch gehalten, weil die Amerikaner uns dabei Vorgaben gemacht haben. Selbstverständlich vollzieht sich gerade jede international relevante Entscheidung im Gegenüber von Entscheidungen des jeweiligen Partners. Dass diese sich nicht ihrerseits auf den demokratischen Willen des eigenen Volkes zuführen lassen können, liegt in der Natur der Sache. Ebenso müssen demokratische Entscheidungen im Angesicht von technischen Entwicklungen getroffen werden, die selbstverständlich nicht demokratisch herbeigeführt und verfügbar sind und deshalb als solche auch nicht verantwortet werden müssen. Auch von daher darf man den Legitimationsanspruch nicht überziehen. Im Übrigen ist eine Kooperationspflicht wie im Telekommunikationsbereich nicht Hinnahme nur des nicht überwindbaren Willens anderer Behörden, sondern in ihr liegt die parlamentarisch demokratische Entscheidung, mit anderen zusammenzuarbeiten. Auch das kann eine Form demokratischer Willensbetätigung sein wenn diese nur hinreichend zurückgebunden ist und sich nicht verselbständigt. Ein wichtiges Element der Ausgestaltung demokratischer Legitimation ist deshalb, dass solche Entscheidungen rückgekoppelt bleiben und auch wieder in Frage gestellt werden können. Es müssen von den Strukturen her Mittel geschaffen werden, solche Übertragungen auf verschiedene Entscheidungszentren sachhaltig stets neu zu prüfen bzw. wirksame Gelegenheiten geschaffen werden, sie erforderlichenfalls zu modifizieren.

Winter: Die Vorträge waren so aufgebaut, dass zunächst die verschiedenen Formen der Verwaltung vorgestellt und dann verfassungsrechtliche und verfassungspolitische Bewertungen vorgenommen wurden, mit Maßstäben wie Legitimation und - wenn dies wirklich ein rechtlicher Maßstab ist - Effizienz. Mir fehlt in dieser Abfolge der Betrachtung ein Zwischenschritt. In diesem wären wohl auch die Bemerkungen von Herrn Raschauer und Herrn Kirchhof anzusiedeln, die aufforderten zu fragen, was am Befund der Formenvielfalt denn eigentlich stört, was erfreut, was ärgert, bevor man dann mit verfassungsrechtlichen Katego- 
rien weitermacht. So wichtig ein solcher Bezug auf praktische Erfahrungen ist, so anspruchsvoll ist er zugleich. Mit spontanen Eindrücken ist es ja nicht getan, vielmehr stellt sich hier ein ganzes Untersuchungsprogramm. Methodisch gesehen sind dabei vor allem die Sozialwissenschaften angesprochen. Zu untersuchen wäre, welche Folgen die Wahrnehmung einer Aufgabe durch eine spezifische Verwaltungsebene hat. Was ist das Besondere, das aus der Kommissionseigenverwaltung, aus der auf Agenturen ausgelagerten Verwaltung, aus der Komitologie, aus der Beibehaltung eines nationalen Vollzugs, aus der Verlagerung auf privatisierte Verwaltung und aus der transnationalen Verwaltung herauskommt? Natürlich stellt sich zugleich die Frage, wie man die Folgen analytisch erfassen kann. Dafür fehlt noch ein Kategoriensystem. Zum Beispiel kann man sich prozedural orientieren und fragen, welche tatsächlichen Strukturen und Einflüsse die Verfahrensnormen auf den verschiedenen Ebenen implementieren oder unterlaufen. Man kann auch inhaltlich fragen, welche diskretionären Elemente in den eher technischen Normen enthalten sind. Ist darin Herrschaftsausübung enthalten? Oder wirken - wie Schelski annahm - nur technische Sachzwänge? Meine Frage an die Referenten ist deshalb: Haben Sie die sozialwissenschaftliche Literatur unter diesem Gesichtspunkt ausgewertet? Gibt es da Mitteilenswertes? Erst wenn man ein praxisgesättigtes Bild von den Folgen der Formenvielfalt hat, kann man zu sinnvollen verfassungsrechtlichen Bewertungen kommen. Dann kann man - hier würde ich mich den Befürwortern der pluralen Legitimation anschließen - mit einem differenzierten Legitimationsmuster aufwarten, das Elemente der Input- und Output-Legitimation verbindet. Übrigens, zum Konzept der Output-Legitimation: $\mathrm{mE}$ ist auch in ihm das Subjekt das Volk und der Gegenstand der Inhalt der Entscheidung, nur ist das Volk hier Instanz der Akzeptabilität, nicht wie bei der Input-Legitimation Akteur bei der Hervorbringung der Entscheidung.

Diskussionsleiter: Mit diesen Fragen sind die Referenten zu ihren abschließenden Stellungnahmen aufgefordert; nach unseren Regeln sind Sie, Herr Groß, der erste.

Groß: Ich habe für mein Referat über die Funktionsgesetze einer Mehrebenenverwaltung ein relativ hohes Abstraktionsniveau zu Grunde gelegt, um gewisse Kernelemente und Koordinationsinstrumente darstellen zu können. Das müsste man in vielen Bereichen stärker ausdifferenzieren. Ich versuche das in den Antworten zum Teil aufzunehmen, aber es ist natürlich in dem Zeitrahmen nur sehr begrenzt möglich. Ich werde zuerst ein paar Einzelfragen behandeln und dann auf die Kernfra- 
gen der demokratischen Legitimation zum Abschluss etwas intensiver eingehen.

Zunächst zur Frage: Benötigt die EU für ihre Verwaltungsorganisation implied powers? Ich glaube, das ist ein falsches Verständnis der Kompetenzregelungen im EU-Vertrag. Wenn dort die Rede ist von „Maßnahmen“, dann ist das von vornherein nicht auf das, was wir als legislative Maßnahmen verstehen, beschränkt, sondern eröffnet grundsätzlich ein weiteres Spektrum, zu dem auch die Errichtung einer Verwaltungsorganisation gehört. Und deswegen ist das Urteil des EuGH zu Art. 95 EGV keine Überraschung. Man braucht das Instrument der implied powers dafür gar nicht.

Zum Thema Subsidiarität: Es ist richtig, dass die Anforderung, dass die Errichtung von Bundesoberbehörden durch Gesetz erfolgen muss, eine kleine Einschränkung ist. Aber wir wissen alle, dass im parlamentarischen Gesetzgebungsverfahren die Exekutive und die Legislative eng zusammengekoppelt sind, so dass die praktische Beschränkungswirkung sehr gering ist. Und die zweite Einschränkung, dass kein Unterbau vorhanden sein darf, ist ja manchmal auch eher eine theoretische Anforderung, wenn man sich das Bundesamt für Migration und Flüchtlinge anschaut, das völlig unkontrovers Dutzende von Außenstellen hat, in denen Asylbewerber angehört werden, und das in jeder einzelnen Kommune Integrationskurse organisiert, nur weil in diesem Fall die Länder die Aufgabe nicht übernehmen wollten. Das ist auch schwer unter Art. 87 Abs. 3 GG zu subsumieren, hat aber bisher erstaunlicher Weise noch kaum Diskussionen hervorgerufen.

Das andere, was ich hier thematisieren will, ist Art. 84 Abs. 1 GG. Richtig ist, dass auf den ersten Blick die neue Fassung in der Tat sogar eine Vereinfachung für den Bundesgesetzgeber ist. Nur dahinter steht natürlich immer die Abweichungsmöglichkeit der Bundesländer. Wenn der Bundesgesetzgeber ein integriertes Konzept verfolgen will, in dem Organisations- und Verfahrenselemente notwendig mit gewissen materiellen Regelungsprogrammen gekoppelt sind, dann muss er auf die jetzt nur noch ausnahmsweise bestehende Möglichkeit der Untersagung der Abweichung zurückgreifen. Und meine Prognose wäre, das ist ja heute Vormittag auch schon angesprochen worden, dass das häufiger vorkommen wird, als man es sich möglicherweise in der Föderalismuskommission gedacht hat.

$\mathrm{Zu}$ den Koordinationstypen: Art. 249 EGV enthält in der Tat kein Äquivalent zu den Verwaltungsvorschriften. Nur wenn man sich anschaut, welchen Umfang und welchen Wirkungsmacht Vollzugsregelungen in der Praxis der EG angenommen haben, ist schon die Frage, ob man gut beraten ist zu sagen, das darf es nicht geben und das ignorieren 
wir, jedenfalls von juristischer Seite her. Viele Stellungnahmen sagen, das ist nur ein politisches Instrument, ein weiches Steuerungsinstrument. Ich habe Schwierigkeiten, wenn ausdrücklich in Sekundärrechtsakten, in Verordnungen oder Richtlinien angeordnet ist, dass solche Richtlinien für nationale Behörde verbindlich sind. Dann müsste man konsequenterweise sagen, diese Regelungen sind ihrerseits primärrechtswidrig. Auch das scheint erstaunlicherweise bisher noch nicht von den Mitgliedstaaten thematisiert worden zu sein.

$\mathrm{Zu}$ den Durchsetzungsmechanismen: Der Bundeszwang ist natürlich ein relativ breites Instrumentarium. Wogegen ich mich gewendet habe, ist die militärische Komponente. Es ist in der Tat schwer vorstellbar, dass man etwa die bayerische Lebensmittelkontrolle durch Bundeswehrsoldaten durchführen lässt, weil sie in einem bestimmten Moment nicht funktioniert. Ob man einen Sparkommissar wirklich auf Art. 37 GG stützen kann, wäre dann eher eine Frage der Bestimmtheit von Verfassungsnormen. Ich würde sagen, das wäre so ein weitgehender Eingriff in die Verfassungsautonomie eines Landes, dass man, wenn man es tatsächlich für notwendig hält, eine solche Institution wohl nur über eine Verfassungsänderung und eine präzisere Grundlage einführen könnte.

$\mathrm{Zu}$ dem Gesamtkomplex der demokratischen Legitimation: Mein Eindruck ist, das ist ja auch in der Diskussion immer wieder deutlich geworden, bei vielen wird Demokratie mit Einfachheit konnotiert. Je einfacher eine bestimmte Struktur ist, desto demokratischer ist sie, weil sie für den Bürger besser durchschaubar ist. Das ist ein nachvollziehbares Bedürfnis. Wenn man es aber mit der von uns beiden geschilderten, sehr komplexen verwaltungsrechtlichen Bestandsaufnahme kontrastiert, stellt sich die Frage, ob diese Konzeption zu Grunde zu legen und die Verwaltungswirklichkeit dann in vielen Teilen als in letzter Konsequenz verfassungswidrig zu qualifizieren ist oder ob man nicht doch das Modell etwas komplexer gestalten muss? Ich will im Grunde genommen das monistische Modell gar nicht verabschieden, sondern ich will seinen Absolutheitsanspruch in Frage stellen und die Regel-Ausnahme-Behauptung, die dahinter steht, dass nur die klassische hierarchische, bürokratische Struktur der Verwaltung mit den personellen Legitimationsstrukturen über die Verwaltungsspitze dem grundlegenden Gebot des Demokratieprinzips insbesondere des Art. 20 GG entspricht, und dass alle abweichenden Gestaltungen entweder unzulässig sind oder jedenfalls einer besonderer verfassungsrechtlichen Rechtfertigung bedürfen. Diese Aussage ist aber keineswegs eine unumstrittene Auslegung des Wortes „Demokratie“, sondern erfordert eine Vielzahl von argumentativen Zwischenschritten, die ihrerseits durchaus kontrovers sind. So suggeriert etwa das angesprochene Legitimationsniveau - das haben 
auch einige angesprochen - quantifizierbare Beiträge. Wenn man aber alle Stellungnahmen des Bundesverfassungsgerichts zum Begriff Legitimationsniveau liest, findet man dort niemals quantifizierbare Aussagen für bestimmte Teilelemente, sondern das ist im Grunde genommen, wenn man es in seiner Funktion analysiert, der Weichmacher, der verhindert, dass das traditionelle Modell überhaupt alles, was von der Bürokratie abweicht, als unzulässig erklärt. Und dann muss man konsequenterweise davon Abschied nehmen, dass bestimmte Legitimationselemente, über deren Koexistenz man sich grundsätzlich einig ist, einen absoluten Wert haben, sondern das ist letztlich eine Gestaltungsfrage, und zwar für den Gesetzgeber. Es geht darum, dass der demokratisch legitimierte Gesetzgeber, dass das Parlament, das nach Art. 20 Abs. 2 GG mit seiner direkten Legitimation im Zentrum einer demokratischen Herrschaft steht, mehr Spielraum bei der Gestaltung auch der Verwaltungsorganisation und der Verwaltungsverfahren hat. Anders und, das habe ich auch gesagt, wahrscheinlich strenger muss man bei der primären Politikgestaltung sein. Bei der Gesetzgebung würde man bestimmte Verflechtungsformen sicher strenger sehen müssen als in der Verwaltung. Aber bei der Verwaltung geht es um einen weiteren Schritt, bei demgrundsätzlich - nicht nur, was die Programmierung, sondern auch, was die Organisation angeht - der gestaltende Zugriff des Gesetzgebers möglich ist.

Aus der Perspektive des Bürgers kann das natürlich im Einzelfall zu Problemen führen. Das habe ich gar nicht bestritten. Die Frage wer verantwortlich ist, muss immer beantwortet werden. Nur gibt es dazu durchaus Beispielsfälle, die wir auch innerstaatlich gelöst haben, wie das Einvernehmen nach $\S 36$ BauGB. Nach außen tritt die Genehmigungsbehörde auf, also etwa das Landratsamt, es ist aber intern die Zustimmung der Gemeinde notwebdig. Bei der Amtshaftung wird dann möglicherweise direkt die Gemeinde verantwortlich gemacht. Bei der Auftragsverwaltung können auch die interne Zuständigkeit, wenn eine Weisung ausgesprochen wird, und die Zuständigkeit nach außen auseinander fallen. Oder noch krasser ist es bei Widerspruchsentscheidungen. Wenn eine Stadt einen Verwaltungsakt erlässt, den das Regierungspräsidium als Widerspruchsbehörde ändert, dann wird trotzdem die Stadt verklagt und muss diesen Verwaltungsakt, der inhaltlich von der Widerspruchsbehörde, einer Landesbehörde, getragen wird, verteidigen. Auch das sind Fälle, wo der Bürger sich fragen könnte, wer verantwortlich ist, wo die nach außen bestehende Verantwortung eine andere ist als die demokratische Innenverantwortung. Ein anderes Beispiel sind Genehmigte Satzungen. Einen Teil der Verantwortung übernimmt die Genehmigungsbehörde, nach außen ist es trotzdem eine gemeindliche Entscheidung. 
Wir kennen solche Fälle also auch im innerstaatlichen Verwaltungsrecht und wir werden auch im europäischen Verwaltungsrecht damit zu recht kommen. Insofern sehe ich auch die Stellungnahmen von ausländischen Regulierungsbehörden im TK-Recht letztlich nicht als neues Problem an. Mir würden als Parallele die Stellungnahmen von Nachbargemeinden im Bauleitplanungsverfahren einfallen, die auch dort innerhalb der Abwägung zu berücksichtigen sind. Folgen hat diese Konstruktion für die gerichtliche Kontrolle. Es wird dann nämlich eine abwägungsähnliche Entscheidungsprärogrative der Verwaltung bestehen, aber wenn eine ausländische Behörde die entsprechende Richtlinie klar missversteht, dann wird man deren Stellungnahme sicher nicht für verbindlich halten.

Eine letzte Stellungnahme vielleicht noch zum Thema Kompromiss. Der Kompromiss als Problem ist sicher auch mit der Frage der Einfachheit von Legitimationszügen verbunden. Ich glaube, wenn solche Kompromisse in Strukturen gefasst werden, in die die Bürger jedenfalls grundsätzlich Vertrauen haben können - und die bundesstaatliche Struktur ist eine solche, in die, jedenfalls nach dem, was man auch aus Umfragen sehen kann, grundsätzlich Vertrauen besteht - und wenn es auch gelingt, die europäischen Verbundstrukturen so in das Bewusstsein der Öffentlichkeit zu bringen, dass die Bürger sehen, dass sie funktionieren, dann sind solche strukturellen Verantwortlichkeitssicherungen wichtiger, als dass man einzelne Personen für konkrete Entscheidungen verantwortlich machen kann.

Pache: Zunächst möchte auch ich allen Diskussionsrednern für ihre Diskussionsbeiträge und Anregungen danken. Auf zahlreiche der Beiträge und Themen, die unsere beiden Referate betrafen, ist bereits mein Mitberichterstatter eingegangen, so dass ich mich etwas mehr auf einzelne thematische Schwerpunkte konzentrieren kann.

Ich möchte zunächst noch einmal - anknüpfend an verschiedene Gesichtspunkte, die Herr Groß bereits angesprochen hat - auf die Fragen zur Legitimation innerhalb der Mehrebenenverwaltung eingehen. Erst einmal also zu der Frage, ob die Forderungen nach demokratischer und nach rechtsstaatlicher Legitimation des Verwaltungshandelns im Mehrebenensystem als rechtliche oder eher als politische Postulate zu verstehen sind, oder konkreter zu der Frage nach einer normativen Grundlage für das Erfordernis demokratischer und rechtsstaatlicher Legitimation der Mehrebenenverwaltung.

Mir ist wichtig zu betonen, dass der Ruf nach demokratischer und rechtsstaatlicher Legitimation der europäischen Integration im allgemeinen und der europäischen Mehrebenenverwaltung im besonderen kei- 
neswegs allein die Artikulation einer rechtspolitischen oder rechtstheoretischen Idealvorstellung oder eines frommen Wunsches darstellt, sondern dass es sich bei dieser Forderung um ein konkret normativ zu verortendes Gebot des Unionsverfassungsrechts handelt.

Die Erforderlichkeit hinreichender demokratischer und rechtsstaatlicher Legitimation der Ausübung von Hoheitsgewalt auf europäischer Ebene und insbesondere der Ausübung von Hoheitsgewalt in der europäischen Mehrebenenverwaltung findet ihre normative Grundlage zum einen in Art. 6 des EU-Vertrages, der die grundlegenden Verfassungsprinzipien der EU anspricht und herausstellt, dass die Union unter anderem auf den Grundsätzen der Demokratie und der Rechtsstaatlichkeit beruht. Hier werden Demokratie und Rechtsstaatlichkeit als zentrale Unionsstrukturelemente primärrechtlich festgeschrieben, und aus diesen in Art. 6 EUV festgelegten Unionsverfassungsgrundsätzen ergibt sich die Erforderlichkeit demokratischer und rechtsstaatlicher Legitimation allen Handelns der EU.

Dieses Legitimationserfordernis ergibt sich darüber hinaus in gleicher Weise rechtsverbindlich daraus, dass es aus der in Art. 6 Abs. 1 Halbsatz 2 des EU-Vertrages ausdrücklich angesprochenen gemeinsamen rechtsstaatlichen und demokratischen Struktur aller Mitgliedstaaten der EU im europäischen Verfassungsverbund folgt und insoweit einen allgemeinen Rechtsgrundsatz des Unionsrechts mit Verfassungsrang bildet.

Alle Ausübung von Hoheitsgewalt auf europäischer Ebene und im Zusammenwirken von europäischen und nationalen Institutionen und Verwaltungen muß also rechtlich zwingend - auf der konkreten normativen Grundlage des Unionsverfassungsrechts und des nationalen Verfassungsrechts der Mitgliedstaaten - demokratisch und rechtsstaatlich strukturiert und legitimiert sein. Problematisch an der normativen Verortung dieser Anforderungen ist allenfalls der geringe Konkretisierungsgrad dieser Forderungen nach demokratischer und rechtsstaatlicher Legitimation im geschriebenen Unionsrecht oder als allgemeiner Rechtsgrundsatz und damit die Bestimmung des konkreten Inhalts der entsprechenden Forderungen.

Diesbezüglich besteht aber kein grundlegender, qualitativer Unterschied zur Art und Weise der Festlegung der entsprechenden Anforderungen des Demokratieprinzips oder des Rechtsstaatsprinzips an die deutsche Staatsgewalt im deutschen Grundgesetz. Auch die staatsstrukturbezogenen Vorgaben des Art. 20 GG bleiben im Verfassungstext eher abstrakt. Ihr Abstraktionsgrad steht aber selbstverständlich nicht ihrer Geltung als verbindliches Verfassungsrecht entgegen, stattdessen macht er lediglich die Ableitung konkreter Anforderungen und Vorgaben des 
Demokratieprinzips und des Rechtsstaatsprinzips für die deutsche Staatsgewalt im Wege der Verfassungsinterpretation erforderlich.

Gleiches gilt für die Anforderungen des Demokratieprinzips und des Rechtsstaatsprinzips an die Ausübung von Hoheitsgewalt auf der Ebene der Europäischen Union und in der europäischen Mehrebenenverwaltung und für die Legitimation dieser Hoheitsgewalt. Ausgangspunkte zur Bestimmung des konkreten Gehalts dieser Anforderungen sind das Demokratieprinzip und das Rechtsstaatsprinzip, wie sie jeweils im EUVertrag und in den gemeinsamen Verfassungstraditionen der Mitgliedstaaten verwurzelt sind. Diese Strukturvorgaben müssen interpretativ konkretisiert werden, aus ihnen sind - wie aus den Staatsstrukturprinzipien im nationalen Verfassungsrecht für die deutsche Staatsgewalt - die konkreten Gehalte der erforderlichen demokratischen und rechtsstaatlichen Legitimation abzuleiten.

In diesem Rahmen spielt dann als ein wichtiges Element für die Bestimmung des eigenständigen Gehalts des europäischen, auf die europäische Mehrebenenverwaltung bezogenen Demokratie- und Rechtsstaatsverständnisses der Ansatz des Grundgesetzes mit seiner Forderung nach repräsentativ-demokratischer Legitimation eine wichtige Rolle. Neben diesen repräsentativ-demokratischen Elementen können jedoch auch andere Vorstellungen, Ansätze und Konzepte von Demokratie, Rechtsstaatlichkeit und Legitimation in die spezifischen Vorgaben für die europäische Mehrebenenverwaltung einfließen.

Für die Legitimation der europäischen Mehrebenenverwaltung kommen ergänzend etwa partizipative Demokratievorstellungen in Betracht, die auf demokratische Teilhaberechte an konkreten Verwaltungsvorgängen abstellen und Legitimation insbesondere durch Verfahrensteilhabe vermitteln und verfahrensbegleitend absichern wollen. Ebenso kommen Elemente all der anderen zwischenzeitlich angesprochenen Legitimationskonzepte und Demokratiemodelle in Europa in Betracht, die den europäischen Demokratiebegriff prägen können, und nur unter angemessener Berücksichtigung dieser unterschiedlichen Vorgaben und Vorstellungen können die Vorgaben des Art. 6 EU-Vertrag für die europäische Mehrebenenverwaltung zutreffend ermittelt werden, kann entschieden werden, wie konkret die europäisch geforderte demokratische und rechtsstaatliche Ausgestaltung europäischen Verwaltens auszusehen hat.

Hinzu kommt, daß Zweifel an der ausschließlichen Eignung repräsentativ-demokratischer Legitimationsansätze zur hinreichenden Legitimation von Herrschaft und Hoheitsgewalt auch im deutschen Staats- und Verfassungsrecht zunehmend Verbreitung finden. Dies zeigt sich etwa bereits am simplen Beispiel der Baugenehmigung, das durch seine ver- 
fahrensrechtliche Verschränkung materieller und prozeduraler gesetzlicher Steuerung, die Einbindung partizipativer Elemente für Grundeigentümer und Betroffene sowie die verfassungsrechtlich garantierten Beteiligungsrechte der kommunalen Selbstverwaltungskörperschaften die Ergänzungsbedürftigkeit oder jedenfalls Modifikationsbedürftigkeit allein repräsentativ-demokratischer Legitimationsmodelle dokumentiert. Auf nationaler wie auf europäischer Ebene sollte man sich in der Auseinandersetzung mit der Art und Weise der Legitimation von Herrschaftsgewalt nicht darauf beschränken, punktuelle Korrekturen und Ergänzungen an grundsätzlich weiterhin ausschließlich monistisch ausgerichteten Legitimationsmodellen vorzunehmen.

Im Interesse einer tatsächlich effektiven, wirkkräftigen Legitimation von Hoheitsgewalt und einer realen Rückkoppelung von Herrschaft an den Willen der Herrschaftsunterworfenen gilt es vielmehr, unterschiedliche Ansätze, Möglichkeiten und Elemente von Legitimationsvermittlung gezielt zur Gewährleistung eines hohen oder zumindest eines hinreichenden Legitimationsniveaus miteinander zu kombinieren. Hier kommen für die Legitimation der europäischen Mehrebenenverwaltung vor allem Elemente der bereits angesprochenen Modelle repräsentativdemokratischer Legitimation, der Legitimation durch Partizipation der Betroffenen oder der Öffentlichkeit oder der output-orientierten Legitimation in Betracht, die Legitimation auch durch die Qualität der Ergebnisse der Ausübung von Hoheitsgewalt vermittelt sieht.

Ein weiterer Punkt, auf den ich eingehen möchte, ist die Frage, wer eigentlich bei output-orientierten Legitimationsmodellen das Legitimationssubjekt sein soll, wessen Legitimation also durch gute Ergebnisse etwa einer Verwaltungstätigkeit bewirkt werden kann. Nach meiner Auffassung ist auch bei output-orientierten Legitimationsvorstellungen das Volk das Legitimationssubjekt, das durch den Zusammenschluß in einem Staat oder auch in einem supranationalen Verbund, der Hoheitsgewalt auszuüben berechtigt ist, allen Hoheitsgewalt ausübenden Staatsorganen oder Organen des supranationalen Verbandes den Auftrag erteilt, diese Aufgaben so gut wie möglich und insbesondere effektiv zu erfüllen. Wenn dieser Auftrag zur Erledigung der wahrzunehmenden Aufgaben dann durch die Organe gut und effektiv erfüllt wird, ist dies eine auf diesen vom Volk erteilten Auftrag zurückführbare Art der Legitimation. Hierin ist die Grundlage und der zentrale Erklärungsansatz output-orientierter Legitimationsmodelle und Legitimationsvorstellungen zu sehen.

Welche dieser vorstellbaren Legitimationsansätze und Legitimationselemente zur demokratischen und rechtsstaatlichen Legitimation der Europäischen Union und der europäischen Mehrebenenverwaltung eingesetzt werden sollen, könnte der europäische Verfassungsgeber durch 
Festlegung entsprechender konkreter Anforderungen entscheiden. Diese legitimationsbezogene Konkretisierung des Demokratie- und des Rechtsstaatsprinzips durch den europäischen Verfassungsgeber ist jedoch bisher noch nicht erfolgt, so dass es gegenwärtig im Rahmen der beschriebenen Kompetenzverteilung für die Übernahme und Ausgestaltung von Verwaltungsaufgaben Aufgabe des europäischen Gesetzgebers ist, die allgemeinen verfassungsrechtlichen Vorgaben des Demokratieund des Rechtsstaatsprinzips an die Legitimation der Verwaltung durch eine demokratische und rechtsstaatliche Ausgestaltung des Verwaltungsverfahrens im europäischen Mehrebenensystem zu verwirklichen.

Bei diesen Vorgaben des Demokratieprinzips und des Rechtsstaatsprinzips an die Ausgestaltung der Legitimationsansätze und Legitimationselemente der europäischen Mehrebenenverwaltung handelt es sich im übrigen deshalb um Optimierungsgebote und nicht um Regeln oder zwingend umzusetzende Vorgaben, weil den verfassungsrechtlichen Geboten an demokratische und rechtsstaatliche Legitimation der Verwaltung auf sehr unterschiedliche Art und Weise und durch viele verschiedene in Betracht kommende Legitimationsbausteine Rechnung getragen werden kann. Unter den zahlreichen in Betracht kommenden Möglichkeiten gilt es, eine dem Auftrag der europäischen Mehrebenenverwaltung in der Sache angemessene und auch praktisch umsetzbare Lösung, die möglichst weitgehend demokratische und rechtsstaatliche Anforderungen erfüllt, zu verwirklichen, und welches diese Lösung ist, hat der Gesetzgeber zu entscheiden.

Was löst daran Beunruhigung aus? Das ist ja die Frage, die mehrfach angesprochen worden ist. Beunruhigung lösen die Weite und Offenheit der primär- oder verfassungsrechtlichen Vorgaben an den Gesetzgeber für die Ausgestaltung der konkreten Art und Weise der demokratischen und rechtsstaatlichen Legitimation der europäischen Mehrebenenverwaltung und der mit dieser Weite der Vorgaben verbundene große gesetzgeberische Gestaltungsspielraum aus. Und vielleicht auch das mangelnde Vertrauen in die Fähigkeit oder den Willen des europäischen Gesetzgebers, diesen Auftrag zur Ausgestaltung der Legitimation der europäischen Mehrebenenverwaltung wirklich so zu erfüllen, dass tatsächlich effektiv Einflussnahme auf, demokratische Teilhabe an und $\mathrm{Zu}$ rechenbarkeit der Entscheidungen der europäischen Verwaltung ermöglicht wird.

Und diese Beunruhigung sowie entsprechende Befürchtungen für die Zukunft sind, so denke ich, durch die bisherige Kooperationsgesetzgebung, durch die bisherige Art und Weise, wie der Gesetzgeber von seinen Gestaltungsmöglichkeiten Gebrauch gemacht hat, auch nur bestätigt worden. Vielleicht auch deshalb, weil bei der Ausgestaltung ganz 
konkreter einzelner Verwaltungsverfahren in einzelnen Sachgebieten das Bewusstsein um die Notwendigkeit der Berücksichtigung grundlegender sachgebietsübergreifender Anforderungen wie eben der Notwendigkeit hinreichender demokratischer Legitimation nicht immer so ausgeprägt vorhanden ist wie bei der abstrakten Betrachtung der europäischen Mehrebenenverwaltung allein unter dem Blickwinkel der Verwirklichung von Demokratie und Rechtsstaatlichkeit. Möglicherweise stehen häufig auch andere Gesichtspunkte wie Effizienz im Sinne einer möglichst leichten oder mit möglichst geringem Verwaltungsaufwand verbundenen Aufgabenwahrnehmung eher im Mittelpunkt des Interesses des europäischen Gesetzgebers.

Gerade auch vor diesem Hintergrund halte ich es für sinnvoll und wichtig, das Problem der demokratischen und rechtsstaatlichen Legitimation der europäischen Mehrebenenverwaltung abstrakt und systematisierend in Angriff zu nehmen. Es bietet sich an, typische Situationen für Kooperationsbedürfnisse zwischen den mitgliedstaatlichen Verwaltungen und der europäischen Verwaltung herauszuarbeiten, diese detailliert zu analysieren und für sie zu versuchen, quasi als Modellregelung ein Konzept der durchzuführenden Art und des Verfahrens der Zusammenarbeit der Verwaltungen, der vorzusehenden Beteiligungsrechte und der Rechtsschutzmöglichkeiten für die konkrete Verwirklichung der verfassungsrechtlichen Gebote an demokratische und rechtsstaatliche Legitimation zu entwickeln.

Es gilt, ebenso wie Regeln des allgemeinen Verwaltungsrechts auch Regeln für ein allgemeines Kooperationsrecht für die europäische Mehrebenenverwaltung zur Verfügung zu stellen. Die Entwicklung eines solchen allgemeinen, demokratischen und rechtsstaatlichen Anforderungen genügenden allgemeinen Kooperationsrechts hätte zur Folge, dass für die Ausgestaltung konkreter Kooperationsverfahren der europäische Gesetzgeber entweder auf die Regeln dieses unter spezifischer Berücksichtigung demokratischer und rechtsstaatlicher Anforderungen entwickelten Koopearationsrechts zurückgriffe oder, im Fall des Abweichens, Begründungsbedarf für alternative Gestaltungsmodelle entstünde, der zumindest eine gewisse Rationalität der Verfahrensgestaltung und eine Auseinandersetzung mit den einschlägigen demokratischen und rechtsstaatlichen Vorgaben gewährleisten könnte.

Den Gedanken, dass Kooperation immer oder zwingend zu Verhandlungslösungen oder Kompromissen führen wird, halte ich so nicht für zwingend, weil Kooperation ja durchaus auch Aufsicht oder eben die Einbindung in feste Entscheidungsstrukturen bedeuten kann. Insofern erscheint am ehesten eine sachgebietsspezifische oder kooperationsformspezifische Differenzierung sinnvoll. Das Effizienzprinzip als Leit- 
maßstab für die Ausgestaltung von Verwaltungsverfahren halte ich nicht für allein auf den Richter oder auf die retrospektive Perspektive beschränkt, sondern es kann auch auf Prognoseentscheidungen bezogen werden, darauf also, wie denn optimal aus ex-ante-Sicht Verfahren oder Verfahrensinstrumente eingesetzt werden können, und genau dies ist ja die Situation, in der der Gesetzgeber versuchen muss, die konkrete Zuweisung von Verwaltungsverantwortung oder die konkrete Gestaltung von Kooperationsverfahren vorzunehmen. Abschließend noch zum Gemeinwohl: Daß es kein abstrakt vorgegebenes europäisches Gemeinwohl im in der Diskussion angesprochenen Sinne gibt, ist völlig klar. Mit dem in den Verwaltungsverfahren zu konkretisierenden Gemeinwohl gemeint ist jeweils der sachgebietsspezifische Verwaltungsauftrag oder die konkrete Verwaltungsaufgabe, die durch die zu vollziehenden Normen der europäischen Verwaltung als verfahrensspezifisches Gemeinwohl vorgegeben wird.

Ich hoffe, damit jedenfalls auf die meisten wesentlichen Anmerkungen und Hinweise eingegangen zu sein, so weit es die Zeit erlaubt hat, und danke noch einmal auch für alle anderen Kommentare und für Ihre Aufmerksamkeit.

Diskussionsleiter: Wir haben einen arbeits- aber auch ertragreichen Nachmittag hinter uns. Das Thema war schwierig es hatte viele Ecken und Kanten. Wir sind sicher nicht an der Lösung aller Fragen angekommen, aber vieles ist klarer geworden. Das verdanken wir in erster Linie den beiden Referaten; das verdanken wir auch denen, die ihre Ideen aus dem Publikum eingebracht haben. Ich darf mich bei Ihnen allen noch mal bedanken und freue mich, Sie heute Abend wieder zu sehen. 